\title{
Propriedades de continuação única para soluções de equações de Schrödinger com interação pontual
}

\author{
Héctor José Cabarcas Urriola
}

\author{
DissERTAÇÃO/TESE APRESENTADA \\ AO \\ Instituto DE MATEMÁticA E EstatísticA \\ DA \\ UniVERSIDADE DE SÃO PAUlO \\ PARA \\ OBTENÇÃ̃ DO TÍTULO \\ $\mathrm{DE}$ \\ Doutor EM CiÊNCIAS
}

\author{
Programa: Matemética \\ Orientador: Prof. Dr. Jaime Angulo Pava
}

Durante o desenvolvimento deste trabalho o autor recebeu auxílio financeiro da CAPES

São Paulo, Junho de 2015 


\title{
Propriedades de continuação única para soluções de equações de Schrödinger com interação pontual
}

\begin{abstract}
Esta versão da dissertação/tese contém as correções e alterações sugeridas pela Comissão Julgadora durante a defesa da versão original do trabalho, realizada em 17/08/2015. Uma cópia da versão original está disponível no Instituto de Matemática e Estatística da Universidade de São Paulo.
\end{abstract}

Comissão Julgadora:

- Prof. Dr. Jaime Angulo Pava - IME-USP

- Prof ${ }^{\mathrm{a}}$. Dr ${ }^{\mathrm{a}}$. Nataliia Goloshchapova - IME-USP

- Prof. Dr. Adán José Corcho Fernandez - UFRJ

- Prof. Dr. Mahendra Prasad Panthee - UNICAMP

- Prof. Dr. Gerson Petronilho - UFSCAR 


\section{Resumo}

Cabarcas Urriola, H. J. Propriedades de continuação única para soluções de equações de Schrödinger com ponto de interação. 2015. 120 f. Tese (Doutorado) - Instituto de Matemática e Estatística, Universidade de São Paulo, São Paulo, 2015.

Neste trabalho, estudamos propriedades de continuação única para as soluções da equação tipo Schrödinger com um ponto interação centrado em $x=0$,

$$
\partial_{t} u=i\left(\Delta_{Z}+V\right) u
$$

onde $V=V(x, t)$ é uma função de valor real e $-\Delta_{Z}$ é o operador escrito formalmente como

$$
-\Delta_{Z}=-\frac{1}{2} \frac{d^{2}}{d x^{2}}+Z \delta_{0}
$$

sendo $\delta_{0}$ a delta de Dirac centrada em zero e $Z$ qualquer número real.

Logo, usamos estes resultados para ver o possível fenômeno de concentração das soluções, que explodem, da equação de tipo Schrödinger não linear com um ponto de interação em $x=0$,

$$
\partial_{t} u=i\left(\Delta_{Z} u+|u|^{\rho-1} u\right),
$$

$\operatorname{com} \rho>5$.

Também, mostramos que para certas condições sobre o potencial dependente do tempo $V$, a equação (1) tem soluções não triviais.

Palavras-chave: Equação de Schrödinger, Propriedade de continuação única, Delta de Dirac. 


\section{Abstract}

Cabarcas Urriola, H. J. Unique continuation properties for solutions of Schrödinger equations with point interaction. 2015. 120 f. Tese (Doutorado) - Instituto de Matemática e Estatística, Universidade de São Paulo, São Paulo, 2015.

In this work, we study unique continuation properties for solutions of the Schrödinger equations with an point interaction centered at $x=0$,

$$
\partial_{t} u=i\left(\Delta_{Z}+V\right) u
$$

where $V=V(x, t)$ is real value function and $-\Delta_{Z}$ is the operator formally written

$$
-\Delta_{Z}=-\frac{1}{2} \frac{d^{2}}{d x^{2}}+Z \delta_{0}
$$

and $\delta_{0}$ is Dirac's delta centered at zero and $Z$ is a real number.

Next, we use these results in order to study the possible profile of the concentration of blow up solutions for the non linear Schrödinger equation with a point interaction at $x=0$,

$$
\partial_{t} u=i\left(\Delta_{Z} u+|u|^{\rho-1} u\right),
$$

with $\rho>5$.

Besides, we show that the equation (2) has non trivial solutions for some conditions on the time dependent potencial $V$.

Keywords: Schrödinger equations, Dirac's delta, Unique continuation. 


\section{Conteúdo}

Lista de Símbolos $\quad$ vii

Introdução $\quad$ ix

1 Preliminares $\quad 1$

1.1 Operador de Schrödinger com ponto de interação . . . . . . . . . . . . . . . 1

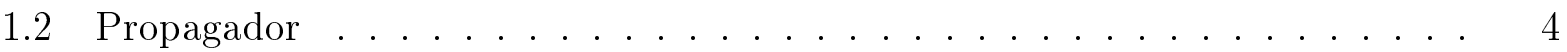

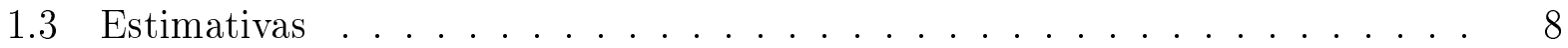

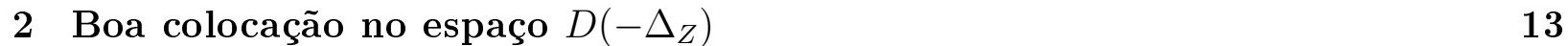

2.1 Estimativas preliminares . . . . . . . . . . . . . . . . 13

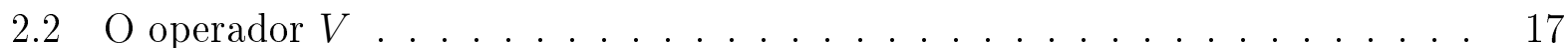

2.3 Existência local e global em $L^{2}(\mathbb{R})$ e $D\left(-\Delta_{Z}\right) \ldots \ldots \ldots \ldots$. . . . . . . 21

3 Propriedades de continuação única $\quad 29$

3.1 Existência e propriedades das funções básicas $\varphi$ e $\psi \ldots$. . . . . . . . . . . 29

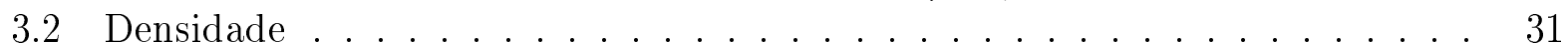

3.3 Operadores simétricos e antisimétricos auxiliares . . . . . . . . . . . 33

3.4 Resultados principais . . . . . . . . . . . . . . . . . 36

4 Aplicações da propriedades de continuação única $\quad \mathbf{5 1}$

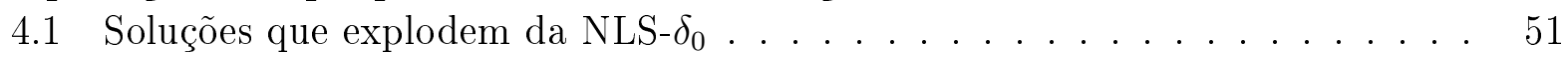

4.2 Concentração das soluções para a equação $4.1 \ldots \ldots$. . . . . . . . . 53

$\begin{array}{lll}5 & \text { Estudos futuros } & 57\end{array}$

A Desigualdades em relação as funções $\varphi$ e $\psi \quad 59$

A.1 Desigualdades em relação a $\varphi \ldots \ldots$. . . . . . . . . . . . 59

A.2 Desigualdades em relação a $\psi \ldots \ldots \ldots$. . . . . . . . . . . 61

B Demonstração da Prop. 3.3 e do Cor. $3.5 \quad 65$

B.1 Demostração da Proposição $3.3 \ldots \ldots \ldots$. . . . . . . . . . 65

B.2 Demonstração do Corolário $3.5 \ldots \ldots \ldots$. . . . . . . . . . . . 66

B.3 Demonstração da identidade $3.17 \ldots \ldots$. . . . . . . . . . . . 69 


\section{Lista de Símbolos}

- $u_{t}(x, t)$ é derivada com respeito à variável temporal $t$

- $H^{m}(\mathbb{R})$, com $m \in \mathbb{Z}^{+}$, são os espaços de Sobolev com $m$ derivadas no sentido das distribuições em $L^{2}(\mathbb{R})$, com norma $\|\cdot\|_{H^{m}}$ definida sobre $H^{m}(\mathbb{R})$,

$$
\|f\|_{H^{m}}^{2}=\sum_{j=0}^{m}\left\|f^{(j)}\right\|_{L^{2}}^{2}
$$

- $\chi_{ \pm}^{0}$ denota a função característica dos conjuntos $\mathbb{R}^{ \pm}$

- $L^{m, \rho}(I)$ é o espaço de Banach de funções de variável real e valor vetorial em $L^{m}(\mathbb{R})$ que são $\rho$ - integráveis, e cuja norma $\|\cdot\|_{L^{m, \rho}}$, se $I=[a, b] \subset \mathbb{R}$, para $u \in L^{m, \rho}(I)$ é

$$
\|u\|_{L^{m, \rho}}=\left\{\int_{I}\left[\int|u(x, t)|^{m} d x\right]^{\frac{\rho}{m}} d t\right\}^{\frac{1}{\rho}}
$$

- $W^{m, p}(\mathbb{R})$, com $m \in \mathbb{Z}^{+}$e $p \in[1,+\infty]$, são os espaços de Sobolev com $m$ derivadas no sentido das distribuições em $L^{p}(\mathbb{R})$

- $\langle x\rangle=\left(1+x^{2}\right)^{\frac{1}{2}}$, para $x \in \mathbb{R}$

- $f(x)=O(g(x))$, notação $O$ grande, significa

$$
|f(x)| \leq M|g(x)|
$$

- $-\Delta_{Z}=-\frac{1}{2} \frac{d^{2}}{d x^{2}}+Z \delta_{0}$,

- A interceç̧ão dos subespaços normados $\left(X,\|\cdot\|_{X}\right)$ e $\left(Y,\|\cdot\|_{Y}\right)$ de um espaço normado $\left(S,\|\cdot\|_{S}\right)$, é de novo um espaço normado, com norma

$$
\|\cdot\|_{X \cap Y}=\max \left\{\|\cdot\|_{X},\|\cdot\|_{Y}\right\}
$$

- O grupo unitário $e^{i t \Delta_{Z}}$ é denotado por $U_{Z}(t)$.

- Para cada $Z \in \mathbb{R}$ e $f \in D\left(-\Delta_{Z}\right)$,

$$
\|f\|_{Z}=\left\|\left(-\Delta_{Z}+m\right)\right\|_{L^{2}},
$$

onde $m=1-\inf \sigma\left(-\Delta_{Z}\right)$ 
- Para $I \times \mathbb{R}, I=[-a, a]$ e $l$, com $0 \leq \frac{1}{2}-\frac{1}{l}<1$ :

$$
\begin{gathered}
\mathfrak{X}(a)=\mathfrak{X}(a, l)=C\left(I ; L^{2}(\mathbb{R})\right) \cap L^{l, \theta}(I), \quad \theta=\frac{4 l}{l-2}, \\
\mathfrak{X}^{*}(a)=\mathfrak{X}^{*}(a, l)=L^{2,1}(I)+L^{l^{\prime}, \theta^{\prime}}(I), \quad \frac{1}{l}+\frac{1}{l^{\prime}}=1, \quad \frac{1}{\theta}+\frac{1}{\theta^{\prime}}=1 .
\end{gathered}
$$

As respectivas normas dos espaços são:

$$
\begin{aligned}
\|u\|_{\mathfrak{X}(a, l)} & =\|u\|_{L^{2, \infty}}+\|u\|_{L^{l, \theta}}, \\
\|u\|_{\mathfrak{X}^{*}(a, l)} & =\inf \left\{\left\|u_{1}\right\|_{L^{2,1}}+\left\|u_{2}\right\|_{L^{l^{\prime}, \theta^{\prime}}}: u=u_{1}+u_{2}\right\} .
\end{aligned}
$$

- Para $I \times \mathbb{R}, I=[-a, a]$ e $l$, com $0 \leq \frac{1}{2}-\frac{1}{l}<1$ :

$$
\mathfrak{J}(a)=\mathfrak{J}(a, l)=\left\{u \in C\left(I ; D\left(-\Delta_{Z}\right)\right): u_{t} \in \mathfrak{X}(a, l)\right\},
$$

e

$$
\mathfrak{J}^{*}(a)=\mathfrak{J}^{*}(a, l)=\left\{u \in C\left(I ; L^{2}(\mathbb{R})\right): u_{t} \in \mathfrak{X}^{*}(a, l)\right\},
$$

cujas normas serão respectivamente:

$$
\|u\|_{\mathfrak{J}(a, l)}=\sup _{t}\|u(t)\|_{Z}+\left\|u_{t}\right\|_{\mathfrak{X}(a, I)},
$$

e

$$
\|u\|_{\mathfrak{J}^{*}(a, l)}=\|u\|_{L^{2, \infty}}+\left\|u_{t}\right\|_{\mathfrak{X}^{*}(a, I)},
$$

- $\mathfrak{B}(\mathfrak{X}(a))$ é o espaço de operadores lineares limitados em $\mathfrak{X}(a)$. 


\section{Introdução}

Em distintas áreas da matemática aparecem resultados que se conhecem com o nome de Propriedade de Continuação Única. Esses resultados nos fornecem condições suficientes não triviais para que duas funções sejam iguais. Em diversos casos, o conjunto de funções com que se trabalha pode ser um espaço linear, assim os teoremas que expressam a propriedade de continuação sempre dão condições suficientes para que a função seja nula. Um exemplo disso é o espaço linear das funções analíticas definidas sobre um subconjunto aberto e conexo $\Omega$ dos complexos. É conhecido que se uma função $f$ se anula sobre uma sequência de pontos de $\Omega$ com ponto de acumulação, então $f$ é identicamente nula sobre $\Omega$.

Dentro das equações diferenciais parciais, propriedades de continuação única podem estar intimamente relacionadas à unicidade de soluções do problema de Cauchy ou associadas ao decaimento no infinito das soluções para certos tempos ou para todo tempo. Um exemplo muito importante e que constitui uma motivação para alguns dos trabalhos de Kenig, Ponce e Vega relacionado à propriedade de continuação única para soluções de equações de tipo Schrödinger é dado em [9]. Este mostra a conexão entre a propriedade de continuação única e a unicidade das soluções em $C\left(\mathbb{R}, L^{2}(\mathbb{R})\right)$ da equação linear de Schrödinger

$$
\left\{\begin{aligned}
\partial_{t} u & =i \partial_{x}^{2} u, & & (x, t) \in \mathbb{R}^{2} \\
u(x, 0) & =h(x), & & x \in \mathbb{R} .
\end{aligned}\right.
$$

Eles usaram o seguinte resultado, conhecido como o Principio de Incerteza de Hardy (ver [19]):

$$
\begin{gathered}
\text { Para } f: \mathbb{R} \rightarrow \mathbb{C} \text {, se } f(x)=O\left(e^{-\frac{x^{2}}{\alpha^{2}}}\right) \text { e sua transformada } \\
\text { de Fourier } \hat{f}(\xi)=O\left(e^{-\frac{4 \xi^{2}}{\beta^{2}}}\right) \text {, com } \alpha, \beta>0 \text { e } 1 / \alpha \beta>1 / 4 \text {, entãa } f \equiv 0 . \\
\text { Também, se } \alpha=\beta=1 \text {, entã } 0 \text { f } f(x)=c e^{-x^{2}} \text {, c constante. }
\end{gathered}
$$

Agora, reescrevendo a solução $u(x, t)=e^{i t \Delta} h(x)$ de $(5)$ e usando o principio de incerteza de Hardy, eles deram uma versão da continuação única para ditas soluções da seguinte maneira:

$$
\begin{gathered}
(\mathrm{A}) \text { Se } u \in C\left(\mathbb{R}, L^{2}(\mathbb{R})\right) \text { é uma solução de }(5) \text { tal que } u(x, 0)=O\left(e^{-x^{2} / \alpha^{2}}\right) \text { e } \\
u(x, T)=O\left(e^{-x^{2} / \beta^{2}}\right) \text {, com } T, \alpha, \beta>0 \text { e } T / \alpha \beta>1 / 4 \text {, então } u \equiv 0 \text {. Também, se } \\
T / \alpha \beta=1 / 4, \text { u tem dado inicial sendo um múltiplo constante de } e^{-\left(1 / \beta^{2}+i / 4 T\right) x^{2}} \text {. }
\end{gathered}
$$

Isto é, uma solução $u(x, t)$ não trivial de (5) não pode decair exponencialmente em dois instantes de tempos diferentes com a ligação em seus decaimentos medidos pela relação $T / \alpha \beta>1 / 4$.

Agora, para a equação diferencial parcial de Schrödinger não linear,

$$
\partial_{t} u=i\left(\partial_{x}^{2} u+|u|^{a-1} u\right), \quad x, t \in \mathbb{R}, \quad a>1 ;
$$


existem soluções da forma $u_{\omega}(x, t)=e^{i \omega t} \phi_{\omega}(x)$, onde $\omega$ é um número real e $\phi_{\omega}: \mathbb{R} \longrightarrow \mathbb{R}$ é uma função $C^{\infty}$. Em particular, existe uma solução desta forma, chamada de ground state, onde $\phi_{\omega}$ é dada explicitamente pela fórmula

$$
\phi_{\omega}(x)=\left\{\frac{(a+1) \omega}{2} \operatorname{sech}^{2}\left(\frac{a-1}{2} \sqrt{\omega} x\right)\right\}^{\frac{1}{a-1}} .
$$

Assim, temos que a solução $u_{\omega}$ correspondente a este perfil tem decaimento exponencial em todo tempo da forma $e^{-\sqrt{\omega}|x|}$. Mais precisamente, tem-se

$$
\left|u_{\omega}(x, t)\right| \leq c(\omega, a) e^{-\sqrt{\omega}|x|}, \quad \forall x, t \in \mathbb{R} .
$$

Assim é natural perguntar-se:

Existem soluções de (6) que tenham um decaimento exponencial mais rápido do que aquele dado pela solução ground state descrita a cima?

Cabe ressaltar que mais rápido significa que a função solução seja $O\left(e^{-\lambda|x|^{p}}\right)$ para algum $\lambda$ suficientemente grande e algum $p \geq 1$. Em esta direção Escauriaza, Kenig, Ponce e Vega em [8] deram uma resposta num contexto mais geral. Eles mostraram que se $u \in C([0, \infty)$ : $\left.L^{2}(\mathbb{R})\right)$ é uma solução forte da equação

$$
\partial_{t} u=i\left(\partial_{x}^{2} u+V(x, t) u\right), \quad x, t \in \mathbb{R}
$$

com potencial dependendo do tempo $V \in L^{\infty}(\mathbb{R} \times[0, \infty))$, sendo a soma de duas funções $V_{1}$ e $V_{2}$ ambas de valor real, $V(x, t)=V_{1}(x, t)+V_{2}(x, t)$, onde $V_{1}$ tem um decaimento lento expressado pela desigualdade,

$$
\left|V_{1}(x, t)\right| \leq \frac{c_{1}}{\left(1+x^{2}\right)^{\alpha}}
$$

para algum $\alpha \geq 0$, e $V_{2}$ tem suporte em $\{(x, t):|x| \geq 1\}$ tal que a derivada radial satisfaz,

$$
-\left(\partial_{r} V_{2}(x, t)\right)^{-} \leq \frac{c_{2}}{|x|^{\beta}}, \quad a^{-}=\min \{a ; 0\}
$$

para algum $\beta \geq 0$. Então, existe uma constante $\lambda_{0}=\lambda_{0}\left(\|V\|_{L^{\infty, \infty}} ; c_{1} ; c_{2} ; \alpha ; \beta\right)>0$ tal que se

$$
\sup _{t>0} \int e^{2 \lambda_{0}|x|^{p}}|u(x, t)|^{2} d x \leq C_{\lambda_{0}}
$$

para algum $p \geq 1$, implica $u \equiv 0$. Isto é, a condição de decaimento sobre $V$ para todo tempo $t \in \mathbb{R}$, implica a existência de um número real positivo $\lambda_{0}$, tal que se $u \not \equiv 0$, então seu decaimento rápido ou exponencial não pode ser da forma $e^{\lambda_{0}|x|^{p}}$, para algum $p \geq 1$. Mais precisamente, sob a condição de que $0 \leq \alpha<1 / 2, \beta=2 \alpha$ e a constante $p$ dada pela fórmula $p=\frac{4-2 \alpha}{3}, p \in(1,4 / 3]$. Esta restrição sobre $p$ faz possível a construção de uma função $\varphi$ de classe $C^{4}$ sobre $\mathbb{R}$, tal que $|x|^{p} \leq M \varphi(x)$ para todo $x \in \mathbb{R}$ e algum $M>0$. Fixando $\lambda>0$ e com ajuda desta função $\varphi$ é possível definir dois operadores $\mathcal{S}$ e $\mathcal{A}$, simétrico e antisimétrico, respectivamente, tal que

$$
e^{\lambda \varphi} i \frac{d^{2}}{d x^{2}} e^{-\lambda \varphi}=\mathcal{S}+\mathcal{A}
$$

onde $\mathcal{S}$ e $\mathcal{A}$ vem dados pela fórmula

$$
\begin{aligned}
& \mathcal{S}=-i \lambda\left(2 \varphi^{\prime} \frac{d}{d x}+\varphi^{\prime \prime}\right), \\
& \mathcal{A}=i\left(\frac{d^{2}}{d x^{2}}+\lambda^{2}\left(\varphi^{\prime}\right)^{2}\right) .
\end{aligned}
$$


Assim, de (9) e usando algumas estimativas que envolvem o comutador associado a $\mathcal{S}$ e $\mathcal{A}$, $[\mathcal{S}, \mathcal{A}]=\mathcal{S} \mathcal{A}-\mathcal{A S}$, em [8] demonstraram que existe uma sequência de tempos, $\left(T_{j}\right)_{j \in \mathbb{Z}^{+}}$ convergindo a infinito, tal que para $f(x, t)=e^{\lambda \varphi(x)} u(x, t)$,

$$
\sup _{j \in \mathbb{Z}^{+}} \int_{T_{1}}^{T_{j}} \int_{\mathbb{R}}|\mathcal{S} f(x, t)|^{2} d x d t \leq \widetilde{C}_{\lambda} .
$$

Como consequência disto, obtiveram a estimativa

$$
\int_{T_{1}}^{T_{j}} \int_{\mathbb{R}} \frac{|f(x, t)|^{2}}{\left(1+x^{2}\right)^{\frac{4-3 p}{2}}} d x d t \leq \widetilde{C}_{\lambda},
$$

uniforme em $j \in \mathbb{Z}^{+}$. Combinando esta última afirmação com o fato de que a norma $L^{2}(\mathbb{R})$ da solução é preservada, é obtida uma contradição, sempre e quando $u_{0} \not \equiv 0$. O caso $\alpha=1 / 2$ é apresentado como uma consequência do caso considerado acima. E por último, quando $\alpha>1 / 2$, seguem uma argumentação similar ao caso $0 \leq \alpha<1 / 2$.

Em particular, se $u$ é uma solução da equação (6) e escolhemos como potencial $V(x, t)=$ $V_{1}(x, t)=|u(x, t)|^{a-1}\left(V_{2} \equiv 0\right)$, com decaimento polinomial do tipo dados em (8), então uma aplicação da propriedade de continuação única descrita acima, mostra que existe um número real positivo $\lambda_{0}$ tal que dita solução não pode decair exponencialmente como $e^{-\lambda_{0}|x|^{p}}$ para algum $p \geq 1$. Estes resultados são exemplos de propriedades de continuação única relacionadas com o decaimento exponencial em todo tempo de existência das soluções das equações diferenciais parciais tipo Schrödinger.

Baseados nesse trabalho de Escauriaza, Kenig, Ponce e Vega em [8] surgiu a seguinte pergunta:

(B) Se consideramos a equação de Schrödinger linear com ponto de interação,

$$
\partial_{t} u=i\left(\Delta_{Z} u+V(x, t) u\right), \quad x, t \in \mathbb{R},
$$

é possivel obter resultados análogos aos afirmados em [8] de tal maneira que as soluções pertençam ao dominio do operador $-\Delta_{Z}, D\left(-\Delta_{Z}\right)$ ?

O operador $-\Delta_{Z}$ é escrito formalmente pela expressão

$$
-\Delta_{Z}=-\frac{1}{2} \frac{d^{2}}{d x^{2}}+Z \delta_{0}
$$

onde $Z \in \mathbb{R}$ e $\delta_{0}$ é a distribuição de Dirac centrada na origem $x=0$. Operadores como estes são conhecidos com o nome de operadores de Schrödinger com um ponto de interação, e podem ser vistos como perturbações do operador de Laplace por medidas suportadas em um conjunto discreto, a saber, perturbações singulares do operador laplaciano. Tais interações foram introduzidas ao longo do tempo por físicos com o fim de estudar problemas em mecânica quântica com interações de alcance-zero (isto é, pontual). O primeiro tratamento matemático destes sistemas físicos foi dado por Berezin e Faddev em [4], usando a teoria de Krein de extensões autoadjuntas de operadores simétricos. Nas preliminares, na seção 1.1, será definido o operador dado em (11) indicando algumas referências que podem ser consultadas para um estudo mais a fundo. Mostra-se também que $-\Delta_{Z}$ é uma extensão autoadjunta do operador laplaciano restrito sobre as funções em $H^{2}(\mathbb{R})$ que anulam-se na origem, e que seu domínio é o espaço

$$
D\left(-\Delta_{Z}\right)=\left\{g \in H^{1}(\mathbb{R}) \cap H^{2}(\mathbb{R} \backslash\{0\}): g^{\prime}\left(0^{+}\right)-g^{\prime}\left(0^{-}\right)=2 Z g(0)\right\} .
$$


Também nas preliminares, na seção 1.2, estudamos o grupo unitário em $L^{2}(\mathbb{R})$ correspondente ao problema de Cauchy associado à equação tipo Schrödinger,

$$
\left\{\begin{array}{rl}
\partial_{t} u & =i \Delta_{Z} u, \quad(x, t) \in \mathbb{R}^{2} \\
u(x, 0) & =h(x), \quad x \in \mathbb{R}
\end{array},\right.
$$

o qual é expressado em termos do grupo correspondente a equação linear de Schrödinger (5).

O modelo linear tipo Schrödinger (10) tem que ser entendido como o seguinte problema de contorno

$$
\left\{\begin{aligned}
\partial_{t} u & =i\left(\frac{1}{2} \partial_{x}^{2}+V(x, t)\right) u, \quad x \neq 0, \\
\lim _{x \rightarrow 0^{+}}[u(x, t)-u(-x, t)] & =0 \\
\lim _{x \rightarrow 0^{+}}\left[\partial_{x} u(x, t)-\partial_{x} u(-x, t)\right] & =2 Z u(0, t), \\
\lim _{x \rightarrow \pm \infty} u(x, t) & =0 .
\end{aligned}\right.
$$

Isto é, uma função $u(x, t)$ solução de (10) satisfaz a equação linear para $x \in \mathbb{R} \backslash\{0\}$, e para cada tempo $t$, contínua em $x=0$, a derivada com respeito a $x$ satisfaz uma condição de salto específica na origem e anula-se no infinito.

Nosso objetivo principal é reunir todos os elementos necessários para responder a questão (B) acima. Um primeiro passo é mostrar a existência de soluções globais não triviais para o problema de Cauchy associados á equação (10) no domínio do operador $-\Delta_{Z}$. Um resultado que segundo nosso conhecimento não existe na literatura. Além disso, mostrar que o conjunto de soluções de equação (10) é não trivial e assim as propriedades de continuação única farão sentido. Também, outra questão para levar em consideração no final das demonstrações dos Teoremas 3.10 e 3.14 é que precisamos da unicidade das soluções do problema (13) abaixo. Por tal motivo, no segundo capítulo estudamos a existência, unicidade e regularidade da solução do problema do valor inicial para equações de Schrödinger com ponto de interação e potencial dependendo do tempo,

$$
\left\{\begin{aligned}
\partial_{t} u & =i\left(\Delta_{Z} u+V(x, t) u\right), \quad t \in \mathbb{R}, \quad x \in \mathbb{R} \\
u(x, 0) & =u_{0}
\end{aligned}\right.
$$

onde $-\Delta_{Z}$ é o operador dado em $(11)$ e $V(x, t)$ é uma função de valor real. Mais precisamente, mostramos que o problema é globalmente bem posto no espaço $L^{2}(\mathbb{R})$ e logo depois, mostramos que se o dado inicial $u_{0}$ pertence ao espaço $D\left(-\Delta_{Z}\right)$, então a solução em $L^{2}(\mathbb{R})$ realmente pertence ao espaço $D\left(-\Delta_{Z}\right)$, para um intervalo de tempo pequeno. Percebemos que a mesma técnica usada por Yajima em [21] pode ser usada para obter nossos resultados associados ao problema de valor inicial. O capítulo 2 está dividido em três seções. Na primeira, apresentamos algumas estimativas tipo $L^{p}-L^{q}$ para o propagador $e^{i t \Delta_{Z}}$ e o operador

$$
\left(S_{Z} u\right)(t)=\int_{0}^{t} e^{i \Delta_{Z}(t-s)} u(s) d s
$$

para $Z \in \mathbb{R}$. Também, definimos uma norma sobre o espaço $D\left(-\Delta_{Z}\right)$ tornando-o um espaço de Banach. Na segunda seção, damos condições sobre a função de valor real $V(x, t)$ e sobre as funções $u(x, t)$, para que o produto $(T u)(x, t)=V(x, t) u(x, t)$ faça sentido. Na terceira seção, reunimos os resultados das seções anteriores para mostrar que a equação integral associada ao problema de valor inicial (13) tem solução e, além disso, mostramos que para certas condições sobre $V(x, t)$, dita solução também é solução de (13). Aqui também mostramos que a norma $L^{2}$ da solução $u(x, t)$ é conservada, com o qual é possível demonstrar a existência global em $L^{2}$. No último teorema deste capítulo (Ver Teorema 2.11) estudamos a existência global no tempo para o espaço $D\left(-\Delta_{Z}\right)$. 
No terceiro capítulo, seção 3.1, começamos com a construção de duas funções auxiliares, que denotamos por $\varphi$ e $\psi$, e foram inicialmente tratadas por Escauriaza, Kenig, Ponce e Vega em [8]. Tais funções, bem como suas primeiras derivadas anulam-se em zero, esta é uma das razões fundamentais que garante que o produto de uma função de suporte compacto que esteja em $D\left(-\Delta_{Z}\right)$ com uma das funções $e^{\lambda \varphi}$ ou $e^{\lambda \psi}$, continue ainda em $D\left(-\Delta_{Z}\right)$. Na seção 3.2 , um resultado importante que conseguimos estabelecer é que o espaço das funções contínuas que pertence a $D\left(-\Delta_{Z}\right)$ que são de classe $C^{\infty}$ para todo $x \in \mathbb{R}$ exceto possivelmente $x \neq 0$ e suporte compacto, é denso em $D\left(-\Delta_{Z}\right)$ (Ver proposição 3.3). Seguidamente, na seção 3.3, com ajuda destas funções conseguimos dois operadores, um simétrico, $\mathcal{S}$, e outro antisimétrico, $\mathcal{A}$, similares aos dados em [8] e que serão tratados explicitamente nesta seção. Notamos que a técnica usada em [8] para obter certas estimativas específicas para $\mathcal{S}$ e $\mathcal{A}$ não podem ser aplicadas em nosso caso por motivo do domínio $D\left(-\Delta_{Z}\right)$. A continuação, demonstramos algumas estimativas, onde é fundamental o carácter simétrico de $\mathcal{S}$ e o antisimétrico de $\mathcal{A}$. Na seção 3.4, conseguimos um primeiro objetivo, enunciamos e demonstramos nossos resultados que expressam as propriedades de continuação única para as soluções da equação (10). Usando a técnica em [8] obtemos os seguintes resultados particulares de continuação única que dão resposta à pergunta $(\mathrm{B})$ estabelecida acima:

Teorema 0.1 Seja $u \in C\left([0, \infty): D\left(-\Delta_{Z}\right)\right) \cap C^{1}\left((0, \infty): L^{2}(\mathbb{R})\right)$ uma solução da equação (10) com potencial $V \in L^{\infty}(\mathbb{R} \times[0, \infty))$ satisfazendo

$$
V(x, t)=V_{1}(x, t)+V_{2}(x, t),
$$

com $V_{j}, j=1,2$ de valor real,

$$
\left|V_{1}(x, t)\right| \leq \frac{c_{1}}{\langle x\rangle^{\alpha}}, \quad 0 \leq \alpha<1 / 2,
$$

e $V_{2}$ com suporte em $\{(x, t):|x| \geq 1\}$ tal que

$$
-\left(\partial_{r} V_{2}(x, t)\right)^{-} \leq \frac{c_{2}}{|x|^{\frac{1+4 \alpha}{3}}}, \quad a^{-}=\min \{a ; 0\} .
$$

Então, existe uma constante $\lambda_{s}=\lambda_{s}\left(\|V\|_{L^{\infty}(\mathbb{R} \times[0, \infty))} ; c_{1} ; c_{2} ; \alpha\right)>0$ tal que,

i. se para $Z \geq 0$, temos

$$
\sup _{t>0} \int_{\mathbb{R}} e^{2 M \lambda_{s}|x|^{p}}|u(x, t)|^{2} d x \leq C_{\lambda_{s}}, \quad p=\frac{4-2 \alpha}{3},
$$

ou

ii. se para $Z<0$, temos

$$
\int_{0}^{\infty}|u(0, t)| d t+\sup _{t>0} \int_{\mathbb{R}} e^{2 M \lambda_{s}|x|^{p}}|u(x, t)|^{2} d x \leq C_{\lambda_{s}}, \quad p=\frac{4-2 \alpha}{3},
$$

ent $\tilde{a} O$

$$
u \equiv 0
$$

Além disso, no Teorema 3.14 determinamos outras condições que implicam a propriedade trivial $u \equiv 0$.

O quarto capítulo é reservado para aplicar as propriedades de continuação única das soluções da equação de tipo Schrödinger não linear com ponto de interação em $x=0$,

$$
\partial_{t} u=i\left(\Delta_{Z} u+|u|^{a-1} u\right), \quad x, t \in \mathbb{R}, \quad a>1 .
$$

Especificamente na seção 4.1 mostramos que existem soluções, em $D\left(-\Delta_{Z}\right)$, que explodem em tempo finito para a equação (19). Na seção 4.2, respondemos à seguinte pergunta: 
Para $a \geq 5$, uma solução $v(x, t)$ que explode em tempo finito da equação (19) pode satisfazer

$$
|v(x, t)| \leq \frac{b}{(1-t)^{\frac{2}{a-1}}} e^{-\lambda|x|^{p}}
$$

para alguns $b, \lambda>0, p \geq 1$ e para todo $t \in(-1,1)$ ?

Nosso resultado no Teorema 4.6 diz que não é possível para $v$ não sendo trivial.

No capítulo 5 propomos alguns problemas que podem ser estudados no futuro.

Nos finalizamos nossa tese com dois apêndices, o apêndice A estabelece propriedades das funções $\varphi$ e $\psi$ definidas no Capítulo 3 . Já o apêndice B estabelece as demonstrações de várias proposições e identidades usadas no Capítulo 3. 


\section{Capítulo 1}

\section{Preliminares}

O objetivo deste capítulo consiste em colecionar definições e resultados (na sua maior parte sem demonstração) que serão a base para o estudo do principio de continuação única e do problema de Cauchy para a equação

$$
\partial_{t} u=i\left(\Delta_{Z}+V(x, t)\right) u \text {. }
$$

Por tal motivo, a primeira seção é dedicada a descrição do operador $-\Delta_{Z}$. Sucessivamente, na segunda seção apresentamos o grupo relacionado com o operador $i \Delta_{Z}$, em termo do o grupo unitário associado à equação de Schrödinger livre, $e^{i t \Delta}$. Por último, damos algumas estimativas que suportarão muitas dos resultados obtidos no Capítulo 2.

\subsection{Operador de Schrödinger com ponto de interação}

Nesta seção vamos dar uma definição matemática do operador escrito formalmente pela expressão

$$
-\Delta_{Z}=-\frac{1}{2} \frac{d^{2}}{d x^{2}}+Z \delta_{0}
$$

onde $Z \in \mathbb{R}$ e $\delta_{0}$ é a distribuição de Dirac centrada na origem $x=0$, a saber, $\left\langle\delta_{0}, \varphi\right\rangle=\varphi(0)$, para $\varphi \in H^{1}(\mathbb{R})$, isto é, $\delta_{0}$ é um elemento de $H^{-1}(\mathbb{R})$. Com esse fim, primeiro faremos uma breve descrição da teoria de Krein de extensão autoadjuntas de operadores simétricos.

Definição 1.1 Sejam A um operador simétrico fechado definido em um espaço de Hilbert e $A^{*}$ o seu adjunto. Os espaços

$$
\begin{aligned}
& \mathcal{L}_{+}=\operatorname{Ker}\left(A^{*}-i\right)=[\operatorname{Ran}(A+i)]^{\perp} \\
& \mathcal{L}_{-}=\operatorname{Ker}\left(A^{*}+i\right)=[\operatorname{Ran}(A-i)]^{\perp}
\end{aligned}
$$

são chamados os espaços de deficiência de $A$ e a dimensão destes são chamados índices de deficiência de $A$, que serão denotados por $n_{+}(A)$ e $n_{-}(A)$, respectivamente.

As isometrias parciais do espaço de deficiência $\mathcal{L}_{+}$no espaço de deficiência $\mathcal{L}_{-}$, isto é, operadores lineares $U: \mathcal{L}_{+} \longrightarrow \mathcal{L}_{-}$cuja restrição ao espaço $[k e r(U)]^{\perp 1}$ são isometrias, nós permitem conhecer a forma das extensões simétricas fechadas de um operador simétrico fechado, e que resumimos no seguinte Teorema (ver [6]):

\footnotetext{
${ }^{1} \mathrm{O}$ espaço $[\operatorname{ker}(U)]^{\perp}$ é chamado o espaço inicial de $U$ e o espaço $\operatorname{Im}(W)$ é chamado o espaço final de $U$.
} 
Teorema 1.2 Seja A um operador simétrico fechado definido em um espaço de Hilbert. Se $W$ é uma isometria parcial com espaço inicial em $\mathcal{L}_{+}$e subespaço final em $\mathcal{L}_{-}$, seja

$$
D_{W}=\left\{f+g+W g: f \in D(A), g \in[\operatorname{ker}(W)]^{\perp}\right\}
$$

e defina o operador $A_{W}$ sobre $D_{W}$ por

$$
A_{W}(f+g+W g)=A f+i g-i W g .
$$

Então, o operador $A_{W}$ é uma extensão simétrica fechada do operador A. Reciprocamente, se um operador $B$ é qualquer extensão simétrica fechada do operador $A$, então existe uma isometria parcial única $W$ tal que $B=A_{W}$, onde o operador $A_{W}$ é como em (1.3). Se a isometria parcial $W$ é tal que tem posto finito, então

$$
n_{ \pm}\left(A_{W}\right)=n_{ \pm}(A)-\operatorname{dim}(\operatorname{Im}(W)) .
$$

O seguinte Teorema nós mostra como o conhecimento de estes espaços de deficiências nós ajudam a determinar se um operador simétrico tem ou não extensões autoadjuntas, e cuja demonstração pode ser encontrada em [6].

Teorema 1.3 Seja A um operador simétrico fechado definido em um espaço de Hilbert com indices de deficiências $n_{ \pm}(A)$.

(a) O operador $A$ é autoadjunto se, e somente se, $n_{ \pm}(A)=0$.

(b) O operador A tem uma extensão autoadjunto se, e somente se, $n_{+}(A)=n_{-}(A)$. Neste caso, o conjunto de extensões está em correspondência natural com o conjunto de isomorfismos de $\mathcal{L}_{+}$sobre $\mathcal{L}_{-}$.

(c) O operadorA é um operador simétrico maximal que não é autoadjunto se, e somente se, ou $n_{+}(A)=0$ e $n_{-}(A)>0$ ou $n_{+}(A)>0$ e $n_{-}(A)=0$.

Um critério simples para determinar se um operador simétrico tem índices de deficiências iguais é o seguinte (Ver [17] para detalhes da demonstração deste Teorema):

Teorema 1.4 (Von Neumann.) Seja A um operador simétrico e suponha que existe uma conjugação Ccom $C: D(A) \rightarrow D(A)$ e $A C=C A$. Então A tem indices de deficiência iguais.

Agora, vamos iniciar com a descrição matemática do operador (1.1). Para isto, consideremos o operador laplaciano $-\Delta$ em $L^{2}(\mathbb{R})$, isto é,

$$
\left\{\begin{array}{l}
-\Delta=-\frac{1}{2} \frac{d^{2}}{d x^{2}} \\
D(-\Delta)=H^{2}(\mathbb{R})
\end{array}\right.
$$

e tomemos a restrição $A$ do operador $-\Delta$ ao espaço $D(A)=\left\{f \in H^{2}(\mathbb{R}): f(0)=0\right\}$, o qual tem a seguintes propriedades:

Lema 1.5 O operador $A$ em $L^{2}(\mathbb{R})$ é fechado, densamente definido e simétrico.

Demonstração. Em [12], pode-se encontrar os detalhes da demonstração.

Agora, é fácil ver que o operador $A$ no Lema 1.5 comuta com a conjugação complexa, $C f=\bar{f}$, pois $A$ é um operador positivo. Então, pelo Teorema 1.4, o operador $A$ tem 
índices deficiências iguais, e consequentemente o Teorema 1.3 implica que $A$ tem extensões autoadjuntas.

Por outro lado, em Albeverio (ver [2]) se mostra que o adjunto $A^{*}$ de $A$ é o operador

$$
\left\{\begin{aligned}
A^{*} & =-\frac{1}{2} \frac{d^{2}}{d x^{2}}, \quad x \neq 0 \\
D\left(A^{*}\right) & =H^{1}(\mathbb{R}) \cap H^{2}(\mathbb{R} \backslash\{0\}) .
\end{aligned}\right.
$$

Logo, pela teoria de equações diferenciais ordinárias, e tendo em conta o domínio $D\left(A^{*}\right)$, do operador autoadjunto $A^{*}$, encontramos que as únicas soluções das equações $A^{*} \phi= \pm i \phi$, com $\phi \in D\left(A^{*}\right)$, são respectivamente $\phi_{ \pm i}(x)=e^{i \sqrt{ \pm i}|x|}$, onde $\operatorname{Im} \sqrt{ \pm i}>0$. Portanto, os índices de deficiências são iguais a 1 e $\mathcal{L}_{+}$é gerado por $\phi_{+i}$ e $\mathcal{L}_{-}$é gerado por $\phi_{-i}$. Logo, as únicas isometrias possíveis são da forma $W_{\lambda} e^{i \sqrt{+i}|x|}=\lambda e^{i \sqrt{-i}|x|}$, para $\lambda \in \mathbb{C}$ e $|\lambda|=1$. De modo, que o Teorema 1.2 nós diz que todas as extensões autoadjuntas de $A$ são da forma

$$
\left\{\begin{aligned}
D\left(A_{\theta}\right) & =\left\{g+c \psi_{+i}+c e^{i \theta} \psi_{-i}: g \in D(A), c \in \mathbb{C}\right\} \\
A_{\theta}\left(g+c \psi_{+i}+c e^{i \theta} \psi_{-i}\right) & =A g+i c \psi_{+i}-i c e^{i \theta} \psi_{-i}
\end{aligned}\right.
$$

onde $\theta \in[0,2 \pi)$ é tal que $\lambda=e^{i \theta} \mathrm{e}$

$$
\psi_{ \pm i}(x)=\frac{i}{2 \sqrt{ \pm i}} e^{i \sqrt{ \pm i}|x|}, \quad \operatorname{Im} \sqrt{ \pm i}>0 .
$$

O seguinte teorema mostra que todas as extensões autoadjuntas $A_{\theta}$ podem parametrizadas em termo do parâmetro $Z$, onde

$$
Z=Z(\theta)=\frac{-2 \cos \left(\frac{\theta}{2}\right)}{\cos \left(\frac{\theta}{2}-\frac{\pi}{4}\right)}
$$

$\operatorname{com} \theta \in[0,2 \pi)$, mas observando que $Z\left((3 \pi / 2)^{-}\right)=+\infty$.

Teorema 1.6 Todas as extensões autoadjuntas $-\Delta_{Z}$ de $A$, onde $-\infty<Z \leq \infty$ são dadas por

$$
\left\{\begin{aligned}
-\Delta_{Z} & =-\frac{1}{2} \frac{d^{2}}{d x^{2}}, \\
D\left(-\Delta_{Z}\right) & =\left\{g \in H^{1}(\mathbb{R}) \cap H^{2}(\mathbb{R} \backslash\{0\}): g^{\prime}\left(0^{+}\right)-g^{\prime}\left(0^{-}\right)=2 Z g(0)\right\} .
\end{aligned}\right.
$$

Se $Z=0$, a extensão autoadjunto $-\Delta_{0}$ corresponde ao operador Laplaciano no espaço $L^{2}(\mathbb{R})$,

$$
\left\{\begin{aligned}
-\Delta & =-\frac{1}{2} \frac{d^{2}}{d x^{2}} \\
D(-\Delta) & =H^{2}(\mathbb{R})
\end{aligned}\right.
$$

No caso $Z=\infty$, a reta real é dividida em dois intervalos $(-\infty, 0)$ e $(0, \infty)$, isto acontece devido a aparição da condição de fronteira do tipo Dirichlet no ponto 0 , isto é

$$
\left\{\begin{aligned}
-\Delta_{\infty} & =\left(-\Delta_{D_{-}}\right) \oplus\left(-\Delta_{D_{+}}\right) \\
D\left(-\Delta_{\infty}\right) & =\left\{g \in H^{1}(\mathbb{R}) \cap H^{2}(\mathbb{R} \backslash\{0\}): g(0)=0\right\}=H_{0}^{2}((-\infty, 0)) \cap H_{0}^{2}((0, \infty)),
\end{aligned}\right.
$$

onde $\left(-\Delta_{D_{ \pm}}\right)$denota o Laplaciano de Dirichlet sobre $(-\infty, 0)$ e $(0, \infty)$, respectivamente, (veja [18], pág. 253), com $D\left(-\Delta_{D_{-}}\right)=H_{0}^{2}((-\infty, 0))$ e $D\left(-\Delta_{D_{+}}\right)=H_{0}^{2}((0, \infty))$. Assim, para todo $Z$ temos

$$
-\Delta_{Z} f(x)=-\frac{1}{2} \frac{d^{2} f(x)}{d x^{2}}, \quad x \neq 0
$$

A continuação dizemos como são os espectros essenciais e discretos do operador $-\Delta_{Z}$; 
Teorema 1.7 Seja $-\infty<Z \leq \infty$. O espectro essencial de $-\Delta_{Z}=-\frac{1}{2} \frac{d^{2}}{d x}+Z \delta_{0}$ é o eixo real não negativo, $\sigma_{\text {ess }}\left(-\Delta_{Z}\right)=[0, \infty)$. Se $-\infty<Z<0,-\Delta_{Z}$ tem exatamente um autovalor simples e negativo, a saber, $\sigma_{p}\left(-\Delta_{Z}\right)=\left\{-\frac{Z^{2}}{4}\right\}$, com $\alpha_{Z}(x)=\sqrt{-\frac{Z}{2}} e^{\frac{1}{2} Z|x|}$ sendo esta autofunção normalizada e estritamente positiva. Se $Z \geq 0$ ou $Z=\infty,-\Delta_{Z}$ não tem autovalores, $\sigma_{p}\left(-\Delta_{Z}\right)=\emptyset$.

Em [2], podemos encontrar as demonstrações dos dois últimos teoremas.

\subsection{Propagador}

Começamos esta seção lembrando um importante teorema na teoria de grupos fortemente contínuos de operadores unitários $\left(C_{0}\right.$ grupo) em um espaço de Hilbert, pois mostra a correspondência entre operadores autoadjuntos e os $C_{0}$ grupos, a saber:

Teorema 1.8 (Stone) Um operador $A$ é o gerador infinitesimal de um $C_{0}$ grupo de operadores unitários sobre um espaço de Hilbert $\mathcal{H}$ se, e somente se, iA é autoadjunto.

Para um estudo a fundo deste resultado pode, por exemplo, consultar [16].

Na seção 1.1 se mostrou que o operador $-\Delta_{Z}$, com $Z \in \mathbb{R}$, é um operador autoadjunto sobre $L^{2}(\mathbb{R})$, assim o Teorema de Stone (Teorema 1.8) implica que $i \Delta_{Z}$, é o gerador infinitesimal de um grupo unitário, que se denotará por $e^{i t \Delta_{Z}}, t \in \mathbb{R}$, e que então satisfaz o seguintes propriedades:

1. Para todo $t \in \mathbb{R}, e^{i t \Delta_{Z}}: L^{2}(\mathbb{R}) \longrightarrow L^{2}(\mathbb{R})$ é uma isometria; o que implica que

$$
\left\|e^{i t \Delta_{Z}} f\right\|_{L^{2}}=\|f\|_{L^{2}}, \quad \forall f \in L^{2}(\mathbb{R})
$$

2. $e^{i t \Delta_{Z}} e^{i t^{\prime} \Delta_{Z}}=e^{i\left(t+t^{\prime}\right) \Delta_{Z}} \operatorname{com}\left(e^{i t \Delta_{Z}}\right)^{-1}=e^{-i t \Delta_{Z}}=\left(e^{i t \Delta_{Z}}\right)^{*}$

3. $e^{i 0 \Delta_{Z}}=1$

4. Fixando $f \in L^{2}(\mathbb{R})$, a função $\Phi_{f}: \mathbb{R} \longrightarrow L^{2}(\mathbb{R})$ definida por $\Phi_{f}(t)=e^{i t \Delta_{z}} f$ é contínua.

No caso particular $Z=0$, se conhece uma fórmula explícita para este grupo unitário em forma de uma convolução,

$$
e^{i t \Delta} u_{0}(x)=\int \frac{e^{\frac{i|x-y|^{2}}{4 t}}}{(4 \pi i t)^{1 / 2}} u_{0}(y) d y, \quad t \neq 0 .
$$

Para o caso $Z \neq 0$, uma representação do grupo unitário $e^{i t \Delta_{Z}}$ foi dado em [14] para $Z>0$ e em [7] para $Z<0$, em termo do grupo unitário $e^{i t \Delta}$. Em ambos os artigos usaram as ideias desenvolvidas em [20]. Aqui se faz uma breve apresentação delas, nós apoiando no feito em [3].

Defina as soluções, $e_{ \pm}(x, \lambda, Z)$, para a equação $\left(-\Delta_{Z}-\lambda^{2} / 2\right) e_{ \pm}=0$ como

$$
e_{ \pm}(x, \lambda, Z)=t_{Z}(\lambda) e^{ \pm i \lambda x} \chi_{ \pm}^{0}+\left(e^{ \pm i \lambda x}+r_{Z}(\lambda) e^{\mp i \lambda x}\right) \chi_{\mp}^{0}, Z, \lambda, x \in \mathbb{R}
$$

onde $\chi_{+}^{0}$ é a função característica de $[0,+\infty), \chi_{-}^{0}$ é a função característica de $(-\infty, 0]$ e

$$
r_{Z}(\lambda)=\frac{Z}{i \lambda-Z}, \quad t_{Z}(\lambda)=1+r_{Z}(\lambda)=\frac{i \lambda}{i \lambda-Z} .
$$


As funções $r_{\lambda}$ e $t_{\lambda}$ são chamados coeficientes de transmissão e reflexão respectivamente e verificam as seguintes relações:

$$
\left|t_{Z}(\lambda)\right|^{2}+\left|r_{Z}(\lambda)\right|^{2}=1
$$

$\mathrm{e}$

$$
t_{Z}(\lambda)=1+r(\lambda)
$$

Agora, para cada $Z \neq 0$ fixado, a família $\left\{\psi_{Z, \lambda}\right\}_{\lambda \in \mathbb{R}}$, onde cada $\psi_{Z, \lambda}$ é definido como

$$
\psi_{Z, \lambda}=\frac{1}{\sqrt{2 \pi}} \begin{cases}e_{+}(x, \lambda, Z), & \text { para } \quad \lambda \geq 0 \\ e_{-}(x, \lambda, Z), & \text { para } \quad \lambda \leq 0\end{cases}
$$

é uma autofunção generalizada do operador $-\Delta_{Z}$ pertencentes ao autovalor $\lambda^{2} / 2$. O Teorema 1.7 implica as seguintes relações de ortogonalidade:

(i) $\int_{\mathbb{R}} \psi_{Z, 0}(x) \overline{\psi_{Z, \lambda}(x)} d x=0$, para todo $\lambda \in \mathbb{R}$;

(ii) $\int_{\mathbb{R}} \psi_{Z, \mu}(x) \overline{\psi_{Z, \lambda}(x)} d x=\delta_{0}(\lambda-\mu)$, para todo $\mu, \lambda \in \mathbb{R}$;

e mais uma, chamada de relação de completamento que depende do sinal do $Z$, isto é,

(iii) para todo $\lambda \in \mathbb{R}$, tem-se

$$
\left\{\begin{aligned}
\psi_{Z, 0}(x) \psi_{Z, 0}(y)+\int_{\mathbb{R}} \psi_{Z, \lambda}(x) \overline{\psi_{Z, \lambda}(y)} d x=\delta_{0}(x-y), & \text { se } \quad Z<0 \\
\int_{\mathbb{R}} \psi_{Z, \lambda}(x) \overline{\psi_{Z, \lambda}(y)} d x=\delta_{0}(x-y), & \text { se } \quad Z>0 .
\end{aligned}\right.
$$

Isto garante a definição de uma transformada de Fourier generalizada

$$
\mathcal{F}(f)(\lambda)=\int_{\mathbb{R}} f(x) \overline{\psi_{Z, \lambda}(x)} d x
$$

e seu adjunto formal

$$
\mathcal{G}(g)(x)=\int_{\mathbb{R}} g(\lambda) \psi_{Z, \lambda}(x) d \lambda .
$$

Assim, de (ii) se obtém imediatamente que $\mathcal{G}$ é a transformada de Fourier inversa, pois

$$
f(\lambda)=f * \delta(\lambda)=\int_{\mathbb{R}} \overline{\psi_{Z, \lambda}(x)} \int_{\mathbb{R}} f(\mu) \psi_{Z, \mu}(x) d \mu d x=\mathcal{F}(\mathcal{G}(g))(\lambda) .
$$

Mais ainda, da relação de completamento (iii) se consegue para toda $f \in L^{2}(\mathbb{R})$ a seguinte expansão (ortogonal) em autofunções de $-\Delta_{Z}$,

$$
f=\left\{\begin{array}{rll}
\left\langle f, \psi_{Z, 0}\right\rangle \psi_{Z, 0}+\int_{\mathbb{R}} \mathcal{F}(f)(\lambda) \psi_{Z, \lambda}(x) d \lambda, & \text { se } & Z<0 \\
\int_{\mathbb{R}} \mathcal{F}(f)(\lambda) \psi_{Z, \lambda}(x) d \lambda, & \text { se } & Z \geq 0
\end{array}\right.
$$

Então, temos do anterior que para $u_{0} \in L^{2}$, a solução do probLema de Cauchy $u \in$ $C\left(\mathbb{R} ; L^{2}(\mathbb{R})\right)$, de

$$
\left\{\begin{aligned}
\partial_{t} u(x, t) & =i \Delta_{Z} u(x, t), \\
u(x, 0) & =u_{0},
\end{aligned}\right.
$$

é dada via o método de separação de variáveis como

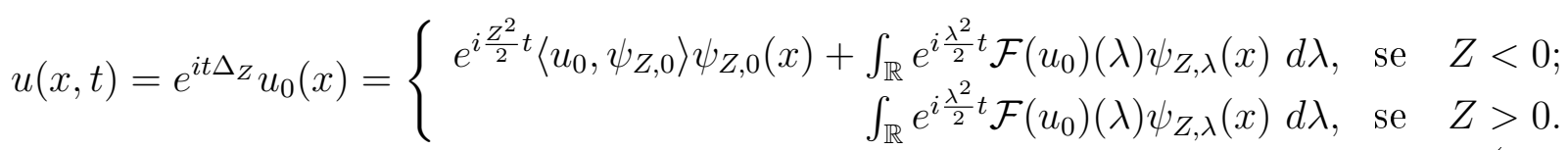


Portanto, obtemos que a projeção espectral contínua $\mathbf{V}_{Z}$, quando $Z \neq 0$, é dada como

$$
\mathbf{V}_{Z}(t) f(x)=\frac{1}{2 \pi} \int_{\mathbb{R}} \int_{0}^{\infty} e^{-i t \frac{\lambda^{2}}{2}}\left[e_{+}(x, \lambda) \overline{e_{+}(y, \lambda)}+e_{-}(x, \lambda) \overline{e_{-}(y, \lambda)}\right] f(y) d \lambda d y
$$

Isto é,

$$
\mathbf{V}_{Z}(t)=\left\{\begin{array}{l}
e^{i t \Delta_{Z}}, \quad \text { se } Z \geq 0 \\
e^{i t \Delta_{Z}}-e^{\frac{1}{2} i t Z^{2}} P_{p} \quad \text { se } \quad Z<0,
\end{array}\right.
$$

onde $P_{p} f=\left\langle f, \psi_{Z, 0}\right\rangle \psi_{Z, 0}$, para toda função $f \in L^{2}(\mathbb{R})$. Seja $P_{p}$ a projeção ortogonal de $L^{2}(\mathbb{R})$ sobre o subespaço espectral discreto de $-\Delta_{Z}, Z<0$. Dessa forma, temos

$$
P_{p}(f)=\frac{\left\langle f, \psi_{Z, 0}\right\rangle}{\left\langle\psi_{Z, 0}, \psi_{Z, 0}\right\rangle} \psi_{Z, 0},
$$

onde $\psi_{Z, 0}=\sqrt{-Z} e^{Z|x|}$, já que $\left\|\psi_{Z, 0}\right\|_{L^{2}(\mathbb{R})}=1$, conseguimos

$$
P_{p} f(x)=\sqrt{-Z} e^{Z|x|} \int_{-\infty}^{+\infty} \sqrt{-Z} e^{Z|y|} f(y) d y
$$

Notemos que o operador $P_{p}$ é um operador limitado de $L^{m^{\prime}}(\mathbb{R})$ em $L^{m}(\mathbb{R})$ e

$$
\left\|P_{p} f\right\|_{L^{m}(\mathbb{R})} \leq\left(\frac{2}{m}\right)^{2 / m}|Z|^{1-2 / m}\|f\|_{L^{m^{\prime}}(\mathbb{R})}
$$

$\operatorname{com} \frac{1}{m}+\frac{1}{m^{\prime}}=1$.

O próximo Lema resume o objetivo desta seção, mas antes de enuncia-lo adotamos a seguinte convenção: para qualquer função $\varphi: \mathbb{R} \rightarrow \mathbb{C}$,

$$
\varphi_{ \pm}^{0}(x)=\varphi(x) \chi_{ \pm}^{0}(x)
$$

e

$$
R \varphi(x)=\varphi(-x)
$$

onde $\chi_{ \pm}^{0}$ são como em (1.13).

Lema 1.9 Seja $v \in L^{1}(\mathbb{R})$. Então

$$
\mathbf{V}_{Z}(t) v(x)=e^{i t \Delta} v(x)+e^{i t \Delta}\left(\rho_{Z} *\left(R v_{+}^{0}+v_{-}^{0}\right)\right)(|x|),
$$

com $\mathbf{V}_{Z}$ como em (1.23) e

$$
\rho_{Z}(x)=\left\{\begin{aligned}
Z e^{Z x} \chi_{+}^{0}(x), & \text { se } \quad Z<0 \\
-Z e^{Z x} \chi_{-}^{0}(x), & \text { se } \quad Z \geq 0
\end{aligned}\right.
$$

onde sua transformada de Fourier é o coeficiente de reflexão $r_{Z}$ definido em (1.14).

Demonstração. Primeiro suponha que supp $v \subset[0,+\infty)$. Da combinação desta hipótese e a fórmula (1.13) temos,

$$
\begin{aligned}
\int_{-\infty}^{+\infty} \overline{e_{+}(y, \lambda)} v(y) d y & =\int_{0}^{+\infty} \overline{t_{Z}(\lambda)} e^{-i \lambda y} v(y) d y \\
& =t_{Z}(-\lambda) \widehat{v}(\lambda)
\end{aligned}
$$


e também

$$
\begin{aligned}
\int_{-\infty}^{+\infty} \overline{e_{-}(y, \lambda)} v(y) d y & =\int_{0}^{+\infty}\left(e^{i \lambda y}+\overline{r_{Z}(\lambda)} e^{-i \lambda y}\right) v(y) d y \\
& =\widehat{v}(-\lambda)+r_{Z}(-\lambda) \widehat{v}(\lambda)
\end{aligned}
$$

Portanto, para $x>0$, usando (1.22), o Teorema de Fubini e as identidades (1.30) e (1.31) obtemos que

$$
\begin{aligned}
\mathbf{V}_{Z}(t) v(x)= & \frac{1}{2 \pi} \int_{0}^{+\infty} e^{-i t \lambda^{2}}\left[e_{+}(x, \lambda) \int_{-\infty}^{+\infty} \overline{e_{+}(y, \lambda)} v(y) d y\right. \\
& \left.+e_{-}(x, \lambda) \int_{-\infty}^{+\infty} \overline{e_{-}(y, \lambda)} v(y) d y\right] d \lambda \\
= & \frac{1}{2 \pi} \int_{0}^{+\infty} e^{-i t \lambda^{2}}\left\{t_{Z}(\lambda) e^{i \lambda x} t_{Z}(-\lambda) \widehat{v}(\lambda)\right. \\
& \left.+\left[e^{-i \lambda x}+r_{Z}(\lambda) e^{i \lambda x}\right]\left[\widehat{v}(-\lambda)+r_{Z}(-\lambda) \widehat{v}(\lambda)\right]\right\} d \lambda \\
= & \frac{1}{2 \pi} \int_{0}^{+\infty} e^{-i t \lambda^{2}}\left[\left|t_{Z}(\lambda)\right|^{2} e^{i \lambda x} \widehat{v}(\lambda)+e^{-i \lambda x} \widehat{v}(-\lambda)+r_{Z}(-\lambda) e^{-i \lambda x} \widehat{v}(\lambda)\right. \\
& \left.+r_{Z}(\lambda) e^{i \lambda x} \widehat{v}(-\lambda)+\left|r_{Z}(\lambda)\right|^{2} e^{i \lambda x} \widehat{v}(\lambda)\right] d \lambda \\
= & \frac{1}{2 \pi} \int_{0}^{+\infty} e^{-i t \lambda^{2}}\left[e^{i \lambda x} \widehat{v}(\lambda)+e^{-i \lambda x} \widehat{v}(-\lambda)+r_{Z}(-\lambda) e^{-i \lambda x} \widehat{v}(\lambda)\right. \\
& \left.+r_{Z}(\lambda) e^{i \lambda x} \widehat{v}(-\lambda)\right] d \lambda \\
= & e^{-i t \Delta} v(x)+e^{-i t \Delta}\left(\rho_{Z} * R v\right)(x) .
\end{aligned}
$$

Analogamente, para $x<0$, conseguimos que

$$
\begin{aligned}
\mathbf{V}_{Z}(t) v(x)= & \frac{1}{2 \pi} \int_{0}^{+\infty} e^{-i t \lambda^{2}}\left\{\left[e^{i \lambda x}+r_{Z}(\lambda) e^{-i \lambda x}\right] t_{Z}(-\lambda) \widehat{v}(\lambda)\right. \\
& \left.\quad+t_{Z}(\lambda) e^{-i \lambda x}\left[\widehat{v}(-\lambda)+r_{Z}(-\lambda) \widehat{v}(\lambda)\right]\right\} d \lambda \\
= & \frac{1}{2 \pi} \int_{0}^{+\infty} e^{-i t \lambda^{2}}\left[e^{i \lambda x} t_{Z}(-\lambda) \widehat{v}(\lambda)+r_{Z}(\lambda) e^{-i \lambda x} t_{Z}(-\lambda) \widehat{v}(\lambda)\right. \\
& \left.\quad+t_{Z}(\lambda) e^{-i \lambda x} \widehat{v}(-\lambda)+t_{Z}(\lambda) e^{-i \lambda x} r_{Z}(-\lambda) \widehat{v}(\lambda)\right] d \lambda \\
= & \frac{1}{2 \pi} \int_{0}^{+\infty} e^{-i t \lambda^{2}}\left[t_{Z}(-\lambda) e^{i \lambda x} \widehat{v}(\lambda)+t_{Z}(\lambda) e^{-i \lambda x} \widehat{v}(-\lambda)\right] d \lambda \\
= & \frac{1}{2 \pi} \int_{-\infty}^{+\infty} e^{-i t \lambda^{2}}\left[1+r_{Z}(-\lambda)\right] e^{i \lambda x} \widehat{v}(\lambda) d \lambda \\
= & e^{i t \Delta} v(x)+e^{i t \Delta}\left(\rho_{Z} * R v\right)(-x)
\end{aligned}
$$

En resumo,

$$
\begin{aligned}
\mathbf{V}_{Z}(t) v(x)= & {\left[e^{i t \Delta} v(x)+e^{i t \Delta}\left(\rho_{Z} * R v\right)(x)\right] \chi_{+}^{0}(x) } \\
& +\left[e^{i t \Delta} v(x)+e^{i t \Delta}\left(\rho_{Z} * R v\right)(-x)\right] \chi_{-}^{0}(x) \\
= & e^{i t \Delta} v(x)+e^{i t \Delta}\left(\rho_{Z} * R v\right)(|x|)
\end{aligned}
$$


Agora, se supp $v \subset(-\infty, 0]$, procedemos de forma análoga ao caso anterior para obter

$$
\mathbf{V}_{Z}(t) v(x)=e^{i t \Delta} v(x)+e^{i t \Delta}\left(\rho_{Z} * v\right)(|x|) .
$$

Então, se $\varphi \in L^{1}(\mathbb{R})$ é qualquer, podemos escrever como suma de duas funções, uma com suporte em $[0, \infty)$ e outra com suporte em $(-\infty, 0]$, a saber,

$$
v=v_{+}^{0}+v_{-}^{0},
$$

com $v_{+}^{0}$ e $v_{-}^{0}$ como em (1.26). Logo, da linearidade do grupo unitário e das equações (1.34) e $(1.35)$, tem-se

$$
\begin{aligned}
\mathbf{V}_{Z}(t) v(x)= & U_{Z}(t)\left(v_{+}^{0}(x)+v_{-}^{0}(x)\right) \\
= & U_{Z}(t) v_{+}^{0}(x)+U_{Z}(t) v_{-}^{0}(x) \\
= & e^{i t \Delta} v_{-}^{0}(x)+e^{i t \Delta}\left(\rho_{Z} * v_{-}^{0}\right)(|x|) \\
& \quad+e^{i t \Delta} v_{+}^{0}(x)+e^{i t \Delta}\left(\rho_{Z} * R v_{+}^{0}\right)(|x|) \\
= & e^{i t \Delta} v(x)+e^{i t \Delta}\left(\rho_{Z} *\left(R v_{+}^{0}+v_{-}^{0}\right)\right)(|x|),
\end{aligned}
$$

como era desejado.

Façamos algumas considerações adicionais respeito ao grupo unitário $U_{Z}(t)=e^{i t \Delta_{Z}}$ sobre uma função do espaço $L^{2}(\mathbb{R})$. Pensemos primeiro em $v \in L^{2}(\mathbb{R})$ como uma função ímpar, então $R v_{+}^{0}(x)=-v_{-}^{0}(x)$, o qual implica imediatamente de (1.28), que $\mathbf{V}_{Z}(t) v(x)=e^{i t \Delta_{z}} v(x)$. Isto nos diz que a forma de agir o grupo $U_{Z}(t)$ sobre as funções ímpares é a mesma que a do grupo $U_{0}(t)$. Por outro lado, se $v$ fosse uma função par, ocorre que $R v_{+}^{0}(x)=v_{-}^{0}(x)$, tornando a expressão (1.28) na forma

$$
\mathbf{V}_{Z}(t) v(x)=e^{i t \Delta} v(x)+2 e^{i t \Delta}\left(\rho_{Z} * v_{-}^{0}\right)(|x|) .
$$

Portanto, devido a que qualquer função $v \in L^{2}(\mathbb{R})$ (geral), pode ser escrita como a soma de uma função par, $\zeta$, e uma ímpar, $\eta,(1.28)$ fica

$$
\mathbf{V}_{Z}(t) v(x)=e^{i t \Delta} v(x)+2 e^{i t \Delta}\left(\rho_{Z} * \zeta_{-}^{0}\right)(|x|) .
$$

A função $\zeta$ sempre a chamaremos a parte par da função $v$.

Agora, já que

$$
\begin{aligned}
e^{i t \Delta}\left(\rho_{Z} * \zeta_{-}^{0}\right)(x) & =\left(e^{-2 \pi^{2} i t \xi^{2}} \widehat{\rho_{Z}}(\xi) \widehat{\zeta_{-}^{0}}(\xi)\right)^{\vee}(x) \\
& =\rho_{Z} *\left(e^{i t \Delta} \zeta_{-}^{0}\right)(x),
\end{aligned}
$$

então a função $e^{i t \Delta}\left(\rho_{Z} * \zeta_{-}^{0}\right)(x)$ é uniformemente contínua e se anula no infinito e sua derivada respeito a $x$ existe e é igual a $\partial_{x} e^{i t \Delta}\left(\rho_{Z} * \zeta_{-}^{0}\right)(x)=Z e^{i t \Delta}\left(\rho_{Z} * \zeta_{-}^{0}\right)(x)$.

Disso, é fácil ver que para $Z \in \mathbb{R}$,

$$
\left\|\partial_{x} e^{i t \Delta}\left(\rho_{Z} * \zeta_{-}^{0}\right)(x)\right\|_{L^{2}} \leq C(Z)\|\zeta\|_{L^{2}} .
$$

\subsection{Estimativas}

Nesta seção vamos apresentar algumas estimativas que serão usadas no capítulo 4 .

Seja $U_{Z}(t)=e^{i t \Delta_{Z}}$ o grupo unitário gerado pela equação de Schrödinger com interação pontual. O caso particular em que $Z=0$, é o grupo de Schrödinger livre, $U_{0}(t)=e^{i t \Delta}$.

Usando o Teorema de interpolação de Riesz- Thorim, se demonstra o seguinte lema (ver [21]): 
Lema 1.10 Seja $2 \leq m \leq \infty$ e $m^{\prime}=\frac{m}{m-1}$ seu expoente conjugado. Então para $t \neq 0$

$$
\left\|U_{0}(t) f\right\|_{L^{m}} \leq(2 \pi|t|)^{-\frac{2}{\theta(m)}}\|f\|_{L^{m^{\prime}}}, \quad \frac{2}{\theta(m)}=\frac{1}{2}-\frac{1}{m}
$$

e $U_{0}(t) u \in C\left(\mathbb{R} \backslash\{0\}: L^{m}(\mathbb{R})\right)$ para cada $u \in L^{m^{\prime}}(\mathbb{R})$.

Uma versão deste lema no caso $Z \neq 0$, é enunciado como segue:

Lema 1.11 Sejam $Z \in \mathbb{R}, 2 \leq m \leq \infty$ e $m^{\prime}=\frac{m}{m-1}$ seu expoente conjugado. Então, para $\mathbf{V}_{Z}$ definida em (1.23), temos

$$
\left\|\mathbf{V}_{Z}(t) f\right\|_{L^{m}} \leq c(2 \pi|t|)^{-\frac{2}{\theta(m)}}\|f\|_{L^{m^{\prime}}}, \quad \frac{2}{\theta(m)}=\frac{1}{2}-\frac{1}{m}
$$

e $\mathbf{V}_{Z}(t) u \in C\left(\mathbb{R} \backslash\{0\}: L^{m}(\mathbb{R})\right)$ para cada $u \in L^{m^{\prime}}(\mathbb{R})$ e c uma constante positiva.

Demonstração. Seja $f \in L^{m^{\prime}}(\mathbb{R})$ e seja $\zeta$ sua parte par, então pela desigualdade de Young $\rho * \zeta_{-} \in L^{m^{\prime}}(\mathbb{R})$ e

$$
\left\|\rho_{Z} * \zeta_{-}\right\|_{L^{m^{\prime}}} \leq\|f\|_{L^{m^{\prime}}}
$$

pois $\left\|\rho_{Z}\right\|_{L^{1}}=1$. Assim, por (1.39) e o Lema 1.10, se obtém que

$$
\begin{aligned}
\left\|\mathbf{V}_{Z}(t) f\right\|_{L^{m}} & \leq\left\|U_{0}(t) f\right\|_{L^{m}}+2\left\|U_{0}(t)\left(\rho_{Z} * \zeta_{-}\right)(|\cdot|)\right\|_{L^{m}} \\
& \leq c(2 \pi|t|)^{-\frac{2}{\theta(m)}}\left(\|f\|_{L^{m^{\prime}}}+2\left\|\rho_{Z} * \zeta_{-}\right\|_{L^{m^{\prime}}}\right) \\
& \leq 3 c(2 \pi|t|)^{-\frac{2}{\theta(m)}}\|f\|_{L^{m^{\prime}}},
\end{aligned}
$$

onde $\frac{2}{\theta(m)}=\frac{1}{2}-\frac{1}{m}$ e $\mathbf{V}_{Z}(t)$ é como em (1.23).

Definamos agora o operador integral

$$
\left(S_{0} u\right)(t)=\int_{0}^{t} U_{0}(t-s) u(s) d s
$$

e vamos introduzirmos os seguintes espaços de Banach sobre $I \times \mathbb{R}, I=[-a, a]$, para o parâmetro $l$, com $0 \leq \frac{1}{2}-\frac{1}{l}<1$ :

$$
\begin{gathered}
\mathfrak{X}(a, l)=C\left(I ; L^{2}(\mathbb{R})\right) \cap L^{l, \theta}(I), \quad \theta=\frac{4 l}{l-2}, \\
\mathfrak{X}^{*}(a, l)=L^{2,1}(I)+L^{l^{\prime}, \theta^{\prime}}(I), \quad \frac{1}{l}+\frac{1}{l^{\prime}}=1, \quad \frac{1}{\theta}+\frac{1}{\theta^{\prime}}=1 .
\end{gathered}
$$

As respectivas normas dos espaços são:

$$
\begin{aligned}
\|u\|_{\mathfrak{X}(a, l)} & =\|u\|_{L^{2, \infty}}+\|u\|_{L^{l, \theta}}, \\
\|u\|_{\mathfrak{X}^{*}(a, l)} & =\inf \left\{\left\|u_{1}\right\|_{L^{2,1}}+\left\|u_{2}\right\|_{L^{l^{\prime}, \theta^{\prime}}}: u=u_{1}+u_{2}\right\} .
\end{aligned}
$$

Com a anterior notação, obtemos as seguintes estimativas (ver [21]):

Lema 1.12 Seja $0 \leq \frac{1}{2}-\frac{1}{l}<1$. Então, para $t \in[-a, a]$

$$
\begin{aligned}
\left\|U_{0}(\cdot) f\right\|_{\mathfrak{X}(a, l)} & \leq C_{1}\|f\|_{L^{2}}, \quad f \in L^{2}(\mathbb{R}) . \\
\left\|S_{0} u\right\|_{\mathfrak{X}(a, l)} & \leq C_{2}\|u\|_{\mathfrak{X}^{*}(a, l)}, \quad u \in \mathfrak{X}^{*}(a, l) .
\end{aligned}
$$

Aqui as constante $C_{1}>0$ é independente de a e f; e $C_{2}>0$ é independente de a e u. 
Agora, vamos mostrar umas estimativas análogas as dadas no Lema anterior, onde o operador envolvido é o operador projeção definido em (1.24), mais exatamente:

Lema 1.13 Sejam $0 \leq \frac{1}{2}-\frac{1}{l}<1$ e $\theta=\frac{4 l}{l-2}$. Então, para $t \in[-a, a]$ e $Z<0$,

$$
\begin{gathered}
\left\|e^{i t \frac{Z^{2}}{2}} P_{p} f\right\|_{\mathfrak{X}(a, l)} \leq c(l, Z)\left((2 a)^{1 / \theta}+1\right)\|f\|_{L^{2}}, \quad f \in L^{2}(\mathbb{R}), \\
\left\|\int_{0}^{t} e^{i(t-s) \frac{Z^{2}}{2}} P_{p} u(s) d s\right\|_{\mathfrak{X}(a, l)} \leq c(l, Z)\left(a^{1 / \theta}+1\right)\|u\|_{\mathfrak{X}^{*}(a, l)}, \quad u \in \mathfrak{X}^{*}(a, l),
\end{gathered}
$$

onde $c(l)$ é uma constante que depende de $l$ e $Z$.

Demonstração. Para $f \in L^{2}(\mathbb{R})$, a desigualdade de Cauchy- Schwart, temos

$$
\begin{aligned}
\left\|e^{i t \frac{Z^{2}}{2}} P_{p} f\right\|_{L^{2, \infty}} & =\left\|\sqrt{|Z|} e^{Z|\cdot|}\right\|_{L^{2}}\left|\int_{-\infty}^{+\infty} \sqrt{|Z|} e^{Z|y|} f(y) d y\right| \\
& \leq\|f\|_{L^{2}}
\end{aligned}
$$

e também,

$$
\begin{aligned}
\left\|e^{i t \frac{Z^{2}}{2}} P_{p} f\right\|_{L^{l, \theta}} & =(2 a)^{\frac{1}{\theta}} \| \sqrt{|Z|} e^{Z|\cdot|}||_{L^{l}}\left|\int_{-\infty}^{+\infty} \sqrt{|Z|} e^{Z|y|} f(y) d y\right| \\
& \leq(2 a)^{\frac{1}{\theta}}\left(\frac{2}{l}\right)^{\frac{1}{l}}|Z|^{\frac{1}{2}-\frac{1}{l}}\|f\|_{L^{2}} .
\end{aligned}
$$

Combinando estas duas últimas estimativas, obtemos (1.47).

Para $u \in \mathfrak{X}^{*}(a, l)$, consideremos $u=u_{1}+u_{2}$, onde $u_{1} \in L^{2,1}(I)$ e $u_{1} \in L^{l, \theta}(I)$, logo

$$
\begin{aligned}
\left\|P_{p} u(s)\right\|_{L^{2}} & \leq\left\|P_{p} u_{1}(s)\right\|_{L^{2}}+\left\|P_{p} u_{2}(s)\right\|_{L^{2}} \\
& \leq\left\|u_{1}(s)\right\|_{L^{2}}+\left(\frac{2}{l}\right)^{\frac{1}{l}}|Z|^{\frac{1}{2}-\frac{1}{l}}\left\|u_{2}(s)\right\|_{L^{l^{\prime}}} .
\end{aligned}
$$

Portanto, para $t \in[-a, a]$

$$
\begin{aligned}
\left\|\int_{0}^{t} e^{i(t-s) \frac{Z^{2}}{2}} P_{p} u(s) d s\right\|_{L^{2, \infty}} & \leq \int_{-a}^{a}\left\|P_{p} u(s)\right\|_{L^{2}} d s \\
& \leq \int_{-a}^{a}\left\|u_{1}(s)\right\|_{L^{2}} d s+\left(\frac{2}{l}\right)^{\frac{1}{l}}|Z|^{\frac{1}{2}-\frac{1}{l}} \int_{-a}^{a}\left\|u_{2}(s)\right\|_{L^{l^{\prime}}} d s \\
& \leq\left\|u_{1}\right\|_{L^{2,1}}+(2 a)^{\frac{1}{\theta}}\left(\frac{2}{l}\right)^{\frac{1}{l}}|Z|^{\frac{1}{2}-\frac{1}{l}}\left\|u_{2}(s)\right\|_{L^{l^{\prime}, \theta^{\prime}}} .
\end{aligned}
$$

Por outro lado,

$$
\left\|P_{p} u(s)\right\|_{L^{l}} \leq\left(\frac{2}{l}\right)^{\frac{1}{l}}|Z|^{\frac{1}{2}-\frac{1}{l}}\left\|u_{1}(s)\right\|_{L^{2}}+\left[\left(\frac{2}{l}\right)^{\frac{1}{l}}|Z|^{\frac{1}{2}-\frac{1}{l}}\right]^{2}\left\|u_{2}(s)\right\|_{L^{l^{\prime}}} .
$$

Então

$$
\begin{aligned}
\left\|\int_{0}^{t} e^{i(t-s) \frac{Z^{2}}{2}} P_{p} u(s) d s\right\|_{L^{l, \theta}} & \leq\left(\int_{-a}^{a}\left(\int_{-a}^{a}\left\|P_{p} u(s)\right\|_{L^{l}} d s\right)^{\theta} d t\right)^{\frac{1}{\theta}} \\
\leq & (2 a)^{\frac{1}{\theta}}\left(\frac{2}{l}\right)^{\frac{1}{l}}|Z|^{\frac{1}{2}-\frac{1}{l}}\left\|u_{1}\right\|_{L^{2,1}} \\
& +\left[(2 a)^{\frac{1}{\theta}}\left(\frac{2}{l}\right)^{\frac{1}{l}}|Z|^{\frac{1}{2}-\frac{1}{l}}\right]^{2}\left\|u_{2}\right\|_{L^{l^{\prime}, \theta^{\prime}}}
\end{aligned}
$$


Combinando as desigualdades (1.49) e (1.50), obtemos a desigualdade (1.48). 


\section{Capítulo 2}

\section{Boa colocação no espaço $D\left(-\Delta_{Z}\right)$}

Neste capítulo abordaremos o estudo da boa colocação local e global, no domínio do operador $-\Delta_{Z}$, do problema de valor inicial,

$$
\left\{\begin{array}{l}
\partial_{t} u=i\left(\Delta_{Z} u+V(x, t) u\right), \quad x \in \mathbb{R}, \quad t \in \mathbb{R} \\
u(x, 0)=u_{0},
\end{array}\right.
$$

onde $V(x, t)$ é uma função de valor real. Primeiro, mostraremos a existência e unicidade da solução do problema (2.1) no espaço $L^{2}(\mathbb{R})$, usando o fato que para certos parâmetros $a, l$ e $\theta \in \mathbb{R}$ o operador

$$
Q_{Z} u(t)=\int_{0}^{t} U_{Z}(t-s) V(x, s) u(s) d s
$$

é um a contração sobre o espaço de Banach $\mathfrak{X}(a, l)=C\left([-a, a] ; L^{2}(\mathbb{R})\right) \cap L^{l, \theta}([-a, a])$. A condição sobre o potencial $V$ será da forma $V \in \mathfrak{M}=L^{p, \alpha}([-a, a])+L^{\infty, \beta}([-a, a])$ (ver Teorema 2.10). Se o potencial $V$ e o dado inicial $u_{0}$ são mais regulares, então mostramos que a solução $u$ correspondente em $L^{2}(\mathbb{R})$, tem a mesma regularidade de seu dado inicial $u_{0}$ (Teorema 2.10). Uma vez mostrada esta última afirmação, é fácil obter que a norma $L^{2}$ da solução $u$ é uma quantidade conservada.

Na seção 2.1, mostramos algumas estimativas que envolvem tanto o grupo unitário $e^{i t \Delta_{Z}}$ quanto o operador dado em (2.5) abaixo que serão úteis para a existência da solução de (2.1). Na seção 2.3 damos sentido à expressão $V(x, t) u(x, t)$, dependendo se vamos mostrar existência de solução no espaço $L^{2}(\mathbb{R})$ ou no espaço $D\left(-\Delta_{Z}\right)$. Finalmente, na seção 2.3, definimos os espaços adequados para aplicar e mostramos a existência de uma solução local em $L^{2}(\mathbb{R})$, logo damos condições sobre $V$ para que dita solução $u$ persista em $D\left(-\Delta_{Z}\right)$, sempre que o dado inicial $u_{0} \in D\left(-\Delta_{Z}\right)$.

\subsection{Estimativas preliminares}

Vamos neste ponto fazer algumas observações análogas à desigualdade (1.45) e que decorrem da forma como é expressado o grupo unitário $e^{i t \Delta_{Z}}$ em termos do grupo unitário $e^{i t \Delta}$.

Lema 2.1 Seja $0 \leq \frac{1}{2}-\frac{1}{l}<1$. Então, para $Z>0$ e $t \in[-a, a]$

$$
\left\|U_{Z}(\cdot) f\right\|_{\mathfrak{X}(a, l)} \leq C(1+\sqrt{2 Z})\|f\|_{L^{2}}, \quad f \in L^{2}(\mathbb{R}),
$$

onde a constante $C>0$ é independente de a e f. Para o caso, $Z<0$ temos

$$
\left\|U_{Z}(\cdot) f\right\|_{\mathfrak{X}(a, l)} \leq C\left(1+\sqrt{2|Z|}+(2 a)^{\frac{1}{\theta}}\left(\frac{2}{l}\right)^{\frac{1}{l}}|Z|^{\frac{1}{2}-\frac{1}{l}}\right)\|f\|_{L^{2}}, f \in L^{2}(\mathbb{R}), t \in[-a, a],
$$


onde $\theta=\frac{4 l}{l-2}$, a constante $C>0$ é independente de a e $f$.

Demonstração. De (1.23), para o caso $Z>0$, temos $\mathbf{V}_{Z}(t)=U_{Z}(t)$. Para mostrar a estimativa (2.3), usamos (1.39), a desigualdade de Hölder, e o Lema (1.12), isto é,

$$
\begin{aligned}
\left\|U_{Z}(\cdot) f\right\|_{\mathfrak{X}(a, l)} & \leq\left\|U_{0}(\cdot) f\right\|_{\mathfrak{X}(a, l)}+\left\|2 U_{0}(\cdot)\left(\rho_{Z} * \zeta_{-}^{0}\right)(|\cdot|)\right\|_{\mathfrak{X}(a, l)} \\
& \leq C\|f\|_{L^{2}}+2 C\left\|\rho_{Z} * \zeta_{-}^{0}\right\|_{L^{2}} \\
& \leq C\|f\|_{L^{2}}+2 C\left\|\rho_{Z}\right\|_{L^{2}}\left\|\zeta_{-}^{0}\right\|_{L^{2}},
\end{aligned}
$$

onde $\zeta_{-}^{0}(x)=(f(x)+f(-x)) \chi_{-}^{0}(x) / 2$. Já que a norma $L^{2}$ da função $\rho_{Z}$ definida em (1.29) é $\sqrt{Z / 2}$, obtemos o resultado desejado. Para $Z<0$, de $(1.23)$ temos $\mathbf{V}_{Z}(t)=-e^{\frac{1}{2} i t Z^{2}} P_{p} f+$ $U_{Z}(t)$, logo de (1.39), a desigualdade de Hölder e o Lema (1.13), obtemos

$$
\begin{aligned}
\left\|U_{Z}(\cdot) f\right\|_{\mathfrak{X}(a, l)} & \leq\left\|e^{\frac{1}{2} i t Z^{2}} P_{p} f\right\|_{\mathfrak{X}(a, l)}+C(1+\sqrt{2|Z|})\|f\|_{L^{2}}, \\
& \leq|Z|\left|\int_{-\infty}^{+\infty} e^{Z|y|} f(y) d y\right|\left\|e^{Z|\cdot|}\right\|_{\mathfrak{X}(a, l)}+C(1+\sqrt{2|Z|})\|f\|_{L^{2}}, \\
& \leq \sqrt{|Z|}\|f\|_{L^{2}}\left(\frac{1}{\sqrt{|Z|}}+(2 a)^{\frac{1}{\theta}}\left(\frac{2}{l|Z|}\right)^{\frac{1}{l}}\right)+C(1+\sqrt{2|Z|})\|f\|_{L^{2}},
\end{aligned}
$$

donde é claro obter (2.4).

A seguir, consideramos o operador

$$
\left(S_{Z} u\right)(t)=\int_{0}^{t} U_{Z}(t-s) u(s) d s,
$$

onde $Z \in \mathbb{R}$. Devido à fórmula dada em (1.39), temos

$$
\left(S_{Z} u\right)(t)= \begin{cases}\left(S_{0} u\right)(t)+\left(S_{0}\left(\rho_{Z} * \zeta_{-}^{0}\right)\right)(|x|, t), & \text { se } Z>0 \\ \int_{0}^{t} e^{\frac{1}{2} i(t-s) Z^{2}}\left(P_{p} u\right)(s) d s+\left(S_{0} u\right)(t)+2\left(S_{0}\left(\rho_{Z} * \zeta_{-}^{0}\right)\right)(|x|, t), & \text { se } Z<0,\end{cases}
$$

onde

$$
\zeta_{-}^{0}(x, t)=\frac{u(x, t)+u(-x, t)}{2} .
$$

O seguinte Lema mostra que $S_{Z}$, fixando o intervalo $[-a, a]$, é um operador limitado de $L^{\infty}\left([-a, a]: L^{2}(\mathbb{R})\right)$.

Lema 2.2 Se $u \in L^{\infty}\left([-a, a]: L^{2}(\mathbb{R})\right)$, então

$$
\left\|S_{Z} u\right\|_{L^{2, \infty}} \leq 2 a\|u\|_{L^{2, \infty}} .
$$

Demonstração. Tomando $K(s, t)=1$ para $0 \leq \pm s \leq \pm t \leq \pm a$ e $K(s, t)=0$ em outro caso, reescrevemos o operador $(2.5)$, como

$$
\left.\left(S_{Z}\right) u(t)=\int_{-\infty}^{+\infty} K_{(} s, t\right) U_{Z}(t-s) u(s) d s .
$$

Logo, a desigualdades de Minkowski implica que

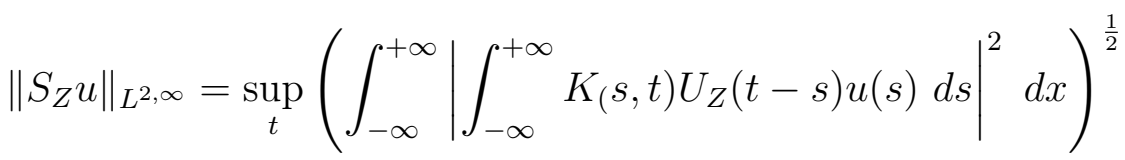

$$
\begin{aligned}
& \left.\leq\left.\sup _{t} \int_{-\infty}^{+\infty}\left(\int_{-\infty}^{+\infty} \mid K_{(} s, t\right) U_{Z}(t-s) u(s)\right|^{2} d x\right)^{\frac{1}{2}} d s \\
& \leq 2 a\|u\|_{L^{2, \infty}}
\end{aligned}
$$


como se queria mostrar.

A continuação vamos mostrar que $S_{Z}$ definido em (2.5) também define, fixado o intervalo $[-a, a]$, um operador limitado do espaço $\mathfrak{X}^{*}(a, l)$ no espaço $\mathfrak{X}(a, l)$. Estes espaços são definidos em (1.44) e (1.43) respectivamente.

Lema 2.3 Para cada $u \in \mathfrak{X}^{*}(a, l)$ e $0 \leq \frac{1}{2}-\frac{1}{l}<1$, temos para $Z>0$

$$
\left\|S_{Z} u\right\|_{\mathfrak{X}(a, l)} \leq c\|u\|_{\mathfrak{X}^{*}(a, l)},
$$

onde c independe de a; mas para $Z<0$, temos

$$
\left\|S_{Z} u\right\|_{\mathfrak{X}(a, l)} \leq c\left((2 a)^{\frac{1}{\theta}}+1\right)\|u\|_{\mathfrak{X}^{*}(a, l)},
$$

onde c depende de $Z$ e independe de a.

Demonstração. Seja $u \in \mathfrak{X}^{*}(a, l)$. Então, $u=u_{1}+u_{2}$, onde $u_{1} \in L^{2,1}(I)$ e $u_{2} \in L^{l^{\prime}, \theta^{\prime}}(I)$ (ver (1.44)), e a parte par de $u$, denotada por $\zeta$, satisfaz a igualdade $\zeta=\zeta_{1}+\zeta_{2}$, com $\zeta_{1}$ e $\zeta_{2}$ as partes pares de $u_{1}$ e $u_{2}$ respectivamente. Pela desigualdade de Young, obtemos que $\rho_{Z} * \zeta_{1} \in L^{2,1}(I), \rho_{Z} * \zeta_{2} \in L^{l^{\prime}, \theta^{\prime}}(I) \mathrm{e}$

$$
\begin{aligned}
\left\|\rho_{Z} * \zeta_{1-}^{0}\right\|_{L^{2,1}} & \leq\left\|u_{1}\right\|_{L^{2,1}}, \\
\left\|\rho_{Z} * \zeta_{2-}^{0}\right\|_{L^{l^{\prime}, \theta^{\prime}}} & \leq\left\|u_{2}\right\|_{L^{l^{\prime} \theta^{\prime}}}
\end{aligned}
$$

Por conseguinte,

$$
\begin{aligned}
\left\|\rho_{Z} * \zeta_{-}^{0}\right\|_{\mathfrak{X}^{*}(a, l)} & \leq\left\|\rho_{Z} * \zeta_{1-}^{0}\right\|_{L^{2,1}}+\left\|\rho_{Z} * \zeta_{2-}^{0}\right\|_{L^{l^{\prime}, \theta^{\prime}}} \\
& \leq\left\|u_{1}\right\|_{L^{2,1}}+\left\|u_{2}\right\|_{L^{l^{\prime} \theta^{\prime}}}
\end{aligned}
$$

Logo, para $Z>0$, a desigualdade (1.46) e (2.6) implicam que

$$
\begin{aligned}
\left\|S_{Z} u\right\|_{\mathfrak{X}(a, l)} & \leq\left\|S_{0} u\right\|_{\mathfrak{X}(a, l)}+2\left\|S_{0}\left(\rho_{Z} * \zeta_{-}^{0}\right)(|\cdot|)\right\|_{\mathfrak{X}(a, l)} \\
& \leq c\|u\|_{\mathfrak{X}^{*}(a, l)}+c\left\|\left(\rho_{Z} * \zeta_{-}^{0}\right)(|\cdot|)\right\|_{\mathfrak{X}^{*}(a, l)} \\
& \leq c\left(\|u\|_{\mathfrak{X}^{*}(a, l)}+\left\|u_{1}\right\|_{L^{2,1}}+\left\|u_{2}\right\|_{L^{l^{\prime} \theta^{\prime}}}\right)
\end{aligned}
$$

donde decorre a desigualdade desejada. Agora, para $Z<0$, por (2.6) e as desigualdades (1.46) e (1.48), obtemos

$$
\begin{gathered}
\left\|S_{Z} u\right\|_{\mathfrak{X}(a, l)} \leq\left\|\int_{0} e^{\frac{1}{2} i(\cdot-s) Z^{2}}\left(P_{p} u\right)(s) d s\right\|_{\mathfrak{X}(a, l)}+\left\|S_{0} u\right\|_{\mathfrak{X}(a, l)}+2\left\|\left(S_{0}\left(\rho_{Z} * \zeta_{-}^{0}\right)\right)(|\cdot|)\right\|_{\mathfrak{X}(a, l)} \\
c(l)\left((2 a)^{\frac{1}{\theta}}+1\right)\|u\|_{\mathfrak{X}^{*}(a, l)}+c\left(\|u\|_{\mathfrak{X}^{*}(a, l)}+\left\|u_{1}\right\|_{L^{2,1}}+\left\|u_{2}\right\|_{L^{l^{\prime} \theta^{\prime}}}\right),
\end{gathered}
$$

procedendo de forma análoga ao caso $Z>0$, obtemos o resultado neste caso.

Agora, definamos a norma $\|f\|_{Z}=\left\|\left(-\Delta_{Z}+m\right) f\right\|_{L^{2}}$, para cada $f \in D\left(-\Delta_{Z}\right)$, onde $m=1-\inf \sigma\left(-\Delta_{Z}\right)$. Esta norma torna ao espaço $D\left(-\Delta_{Z}\right)$ em um espaço de Banach. Do Teorema 1.7, observamos que

$$
m=\left\{\begin{aligned}
1, & \text { se } \quad Z \geq 0 \\
1+\frac{Z^{2}}{4}, & \text { se } \quad Z<0
\end{aligned}\right.
$$

Logo, em qualquer caso $m \geq 1$. Assim, do fato que o operador $-\Delta_{Z}$ é autoadjunto e limitado inferiormente, temos a seguintes relações:

$$
\begin{aligned}
\left\langle\left(-\Delta_{Z}+m\right) f, f\right\rangle & \geq \inf \left\{\sigma\left(-\Delta_{Z}\right)\right\}\|f\|_{L^{2}}^{2}+m\|f\|_{L^{2}}^{2}=\|f\|_{L^{2}}^{2}, \\
\left\|-\Delta_{Z} f\right\|_{L^{2}} & \leq(1+m)\|f\|_{Z} .
\end{aligned}
$$


Assim, vamos considerar os seguintes espaços de Banach:

$$
\mathfrak{J}(a)=\mathfrak{J}(a, l)=\left\{u \in C\left(I ; D\left(-\Delta_{Z}\right)\right): u_{t} \in \mathfrak{X}(a, l)\right\},
$$

e

$$
\mathfrak{J}^{*}(a)=\mathfrak{J}^{*}(a, l)=\left\{u \in C\left(I ; L^{2}(\mathbb{R})\right): u_{t} \in \mathfrak{X}^{*}(a, l)\right\},
$$

cujas normas serão respectivamente:

$$
\|u\|_{\mathfrak{J}(a, l)}=\sup _{t}\|u(t)\|_{Z}+\left\|u_{t}\right\|_{\mathfrak{X}(a, I)}
$$

e

$$
\|u\|_{\mathfrak{J}^{*}(a, l)}=\|u\|_{L^{2, \infty}}+\left\|u_{t}\right\|_{\mathfrak{X}^{*}(a, I)},
$$

com $\mathfrak{X}(a, l)$ e $\mathfrak{X}^{*}(a, l)$ definidos em $(1.43)$ e (1.44).

Agora, mostraremos que para $l$ satisfazendo $0 \leq \frac{1}{2}-\frac{1}{l}<1, S_{Z}$ definido em (2.5), é também um operador limitado do espaço $J^{*}(a, l)$ em $J(a, l)$.

Lema 2.4 Se $u \in \mathfrak{J}^{*}(a, l)$ e $0 \leq \frac{1}{2}-\frac{1}{l}<1$. Então, para $Z>0$

$$
\left\|S_{Z} u\right\|_{\mathfrak{J}(a, l)} \leq C(Z)(1+a)\|u\|_{\mathfrak{J}^{*}(a, l)},
$$

e para $Z<0$

$$
\left\|S_{Z} u\right\|_{\mathfrak{J}(a, l)} \leq C(Z)\left(a+(2 a)^{\frac{1}{\theta}}+1\right)\|u\|_{\mathfrak{J}^{*}(a, l)},
$$

onde $C(Z)$ independem de a e u

Demonstração. Notemos que para $t \in I_{1}=(-a, a)$,

$$
\begin{aligned}
\frac{d}{d t} S_{Z} u(t) & =\frac{d}{d t}\left[U(t) \int_{0}^{t} U_{Z}(-s) u(s) d s\right] \\
& =i \Delta_{Z} U(t) \int_{0}^{t} U_{Z}(-s) u(s) d s+U(t) U(-t) u(t) \\
& =i \Delta_{Z} S_{Z} u(t)+u(t),
\end{aligned}
$$

e

$$
\begin{aligned}
u(t)-U_{Z}(t) u(0) & =\int_{0}^{t} \frac{d}{d s} U_{Z}(t-s) u(s) d s \\
& =\int_{0}^{t}\left[U_{Z}(t-s) u_{t}(s)-i \Delta_{Z} U_{Z}(t-s) u(s)\right] d s \\
& =S_{Z} u_{t}(t)-i \Delta_{Z} S_{Z} u(t)=S_{Z} u_{t}(t)+\left[u(t)-\frac{d}{d t} S_{Z} u(t)\right] .
\end{aligned}
$$

Logo, simplificando, obtemos

$$
\frac{d}{d t} S_{Z} u(t)=S_{Z} u_{t}(t)+U_{Z}(t) u(0) .
$$

Das identidades (2.20) e (2.22), e das estimativas (2.7) e (2.10), temos para $Z>0$,

$$
\begin{aligned}
\sup _{t}\left\|S_{Z} u(t)\right\|_{Z} & \leq \sup _{t}\left\|\Delta_{Z} S_{Z} u(t)\right\|_{L^{2}}+m\left\|S_{Z} u\right\|_{L^{2, \infty}} \\
& \leq \sup _{t}\left\|\frac{d}{d t} S_{Z} u(t)\right\|_{L^{2}}+(1+2 a m)\|u\|_{L^{2, \infty}} \\
& \leq\left\|S_{Z} u_{t}\right\|_{L^{2, \infty}}+\left\|U_{Z}(\cdot) u(0)\right\|_{L^{2, \infty}}+(1+a m)\|u\|_{L^{2, \infty}} \\
& \leq\left\|S_{Z} u_{t}\right\|_{\mathfrak{X}(a, l)}+2(1+a m)\|u\|_{L^{2, \infty}} \\
& \leq c\left\|u_{t}\right\|_{\mathfrak{X}^{*}(a, l)}+2(1+a m)\|u\|_{L^{2, \infty}} .
\end{aligned}
$$


Assim, usando esta última desigualdade e (2.3), conseguimos que

$$
\begin{aligned}
\left\|S_{Z} u\right\|_{\mathfrak{J}(a, l)} & =\sup _{t}\left\|S_{Z} u(t)\right\|_{Z}+\left\|\frac{d}{d t} S_{Z} u\right\|_{\mathfrak{X}(a, I)} \\
& \leq c(1+a m)\left\|u_{t}\right\|_{\mathfrak{J}^{*}(a, l)}+\left\|S_{Z} u_{t}\right\|_{\mathfrak{X}(a, I)}+\left\|U_{Z}(\cdot) u(0)\right\|_{\mathfrak{X}(a, I)} \\
& \leq c(1+a m)\left\|u_{t}\right\|_{\mathfrak{J}^{*}(a, l)}+c\left\|u_{t}\right\|_{\mathfrak{X}^{*}(a, I)}+C(Z)\|u(0)\|_{L^{2}} .
\end{aligned}
$$

Logo, manipulando as constantes em (2.23) é fácil obter a estimativa (2.18). Para o caso $Z<0$, um procedimento análogo ao caso $Z>0$, e usando as desigualdades (2.4) e (2.11), obtemos

$$
\begin{aligned}
\left\|S_{Z} u\right\|_{\mathfrak{J}(a, l)} & =\sup _{t}\left\|S_{Z} u(t)\right\|_{Z}+\left\|\frac{d}{d t} S_{Z} u\right\|_{\mathfrak{X}(a, I)} \\
& \leq c\left((2 a)^{\frac{1}{\theta}}+1\right)\left\|u_{t}\right\|_{\mathfrak{X}^{*}(a, l)}+(1+a m)\|u\|_{L^{2, \infty}}+c\left((2 a)^{\frac{1}{\theta}}+1\right)\|u\|_{L^{2, \infty}}
\end{aligned}
$$

donde obtemos a estimativa (2.19).

\subsection{O operador $V$}

Nesta seção vamos dar sentido á expressão $V(x, t) u(x, t)$. No Lema 2.5 mostramos que $V(x, t) u(x, t)$ é um operador linear limitado do espaço $\mathfrak{X}(a, l)$ no espaço $\mathfrak{X}^{*}(a, l)$, definidos em (1.43) e (1.44). No Lema 2.7 mostramos que $V(x, t) u(x, t)$ é um operador contínuo do espaço $\mathfrak{J}(a, l)$ no espaço $\mathfrak{J}^{*}(a, l)$, definidos em $(2.14)$ e (2.15). Estes resultados são usados para mostrar a existência de soluções para $(2.1)$ no espaço $L^{2}(\mathbb{R})$ e no domínio do operador $-\Delta_{Z}, D\left(-\Delta_{Z}\right)$, respectivamente.

Lema 2.5 Seja $V \in L^{p, \alpha}(I)+L^{\infty, \beta}(I)$ para alguns $p \geq 1, \alpha \geq 1, \beta>1$, satisfazendo a relaçãa $0 \leq \frac{1}{\alpha}<1-\frac{1}{2 p}$. Então

$$
\|V u\|_{\mathfrak{X}^{*}(a, q)} \leq(2 a)^{\gamma}\|V\|_{\mathfrak{M}}\|u\|_{\mathfrak{X}(a, q)}
$$

onde $q=\frac{2 p}{p-1}, 2 a<1, \gamma=\min \left\{1-\frac{1}{\beta}, 1-\frac{1}{2 p}-\frac{1}{\alpha}\right\} e$

$$
\|V\|_{\mathfrak{M}}=\inf \left\{\left\|V_{1}\right\|_{L^{p, \alpha}}+\left\|V_{2}\right\|_{L^{\infty, \beta}, \beta}: V=V_{1}+V_{2}\right\} .
$$

Demonstração. Tome $\theta=\theta(q)=\frac{4 q}{q-2}$. Então $0 \leq \frac{1}{2}-\frac{1}{q}<1, \frac{1}{q^{\prime}}-\frac{1}{q}=\frac{1}{p}$, e $1-\frac{1}{2 p}=\frac{1}{\theta^{\prime}}-\frac{1}{\theta}$. Logo, se $u \in \mathfrak{X}(a, q)$, a desigualdade de Hölder na variável espacial $x$ mostra,

$$
\begin{aligned}
\left\|V_{1} u\right\|_{L^{q^{\prime}, \theta^{\prime}}} & \leq\left(\int_{I}\left[\int_{-\infty}^{+\infty}\left|V_{1}(x, t) u(x, t)\right|^{q^{\prime}} d x\right]^{\frac{\theta^{\prime}}{q^{\prime}}} d t\right)^{\frac{1}{\theta^{\prime}}} \\
& \leq\left(\int_{I}\left[\left(\int_{-\infty}^{+\infty}\left|V_{1}(x, t)\right|^{p} d x\right)^{\frac{q^{\prime}}{p}}\left(\int_{-\infty}^{+\infty}|u(x, t)|^{q^{\prime}(q-1)} d x\right)^{\frac{1}{q-1}}\right]^{\frac{\theta^{\prime}}{q^{\prime}}} d t\right)^{\frac{1}{\theta^{\prime}}} \\
& =\left(\int_{I}\left\|V_{1}(t)\right\|_{L^{p}}^{\theta^{\prime}}\|u(t)\|_{L^{q}}^{\theta^{\prime}} d t\right)^{\frac{1}{\theta^{\prime}}} .
\end{aligned}
$$

O expoente $\theta / \theta^{\prime}$, tem expoente conjugado $2 p \theta^{\prime} /(2 p-1)$, assim, novamente a desigualdade de Hölder, mas esta vez com respeito à variável temporal $t$, implica que

$$
\left\|V_{1} u\right\|_{L^{q^{\prime}, \theta^{\prime}}} \leq\left(\int_{I}\left\|V_{1}(t)\right\|_{L^{p}}^{\frac{2 p}{2 p-1}} d t\right)^{\frac{2 p-1}{2 p}}\left(\int_{I}\|u(t)\|_{L^{q}}^{\theta} d t\right)^{\frac{1}{\theta}} .
$$


Da hipótese $\frac{1}{\alpha}<\frac{2 p-1}{2 p}$ e outra aplicação da desigualdade de Hölder, tem-se

$$
\left\|V_{1} u\right\|_{L^{q^{\prime}, \theta^{\prime}}} \leq(2 a)^{1-\frac{1}{2 p}-\frac{1}{\alpha}}\left\|V_{1}\right\|_{L^{p, \alpha}}\|u\|_{L^{q, \theta}} .
$$

Por outro lado,

$$
\begin{aligned}
\left\|V_{2} u\right\|_{L^{2,1}} & =\int_{I}\left(\int_{-\infty}^{+\infty}\left|V_{2}(x, t) u(x, t)\right|^{2} d x\right)^{1 / 2} d t \\
& \leq \int_{I}\left\|V_{2}(t)\right\|_{L^{\infty}}\left(\int_{-\infty}^{+\infty}|u(x, t)|^{2} d x\right)^{1 / 2} d t \\
& \leq\|u\|_{L^{2, \infty}}\left(\int_{I}\left\|V_{2}(t)\right\|_{L^{\infty}}^{\beta} d t\right)^{1 / \beta}(2 a)^{1-\frac{1}{\beta}} .
\end{aligned}
$$

Portanto, $V u \in \mathfrak{X}^{*}(a, q) \mathrm{e}$

$$
\begin{aligned}
\|V u\|_{\mathfrak{X}^{*}(a, q)} & \leq\left\|V_{2} u\right\|_{L^{2,1}}+\left\|V_{1} u\right\|_{L^{q^{\prime}, \theta^{\prime}}} \\
& \leq\|u\|_{L^{2, \infty}}\left\|V_{2}\right\|_{L^{\infty}, \beta}(2 a)^{1-\frac{1}{\beta}}+(2 a)^{1-\frac{1}{2 p}-\frac{1}{\alpha}}\left\|V_{1}\right\|_{L^{p, \alpha}}\|u\|_{L^{q, \theta}} \\
& \leq(2 a)^{\gamma}\left(\|u\|_{L^{2, \infty}}\left\|V_{2}\right\|_{L^{\infty, \beta}}+\left\|V_{1}\right\|_{L^{p, \alpha}}\|u\|_{L^{q, \theta}}\right)
\end{aligned}
$$

onde $\gamma=\min \left\{(2 a)^{1-\frac{1}{\beta}}, 1-\frac{1}{2 p}-\frac{1}{\alpha}\right\}$, pois $2 a<1$. Já que esta desigualdade vale para toda representação de $V$, obtemos o resultado desejado.

Lema 2.6 Seja $V \in L^{p}(\mathbb{R})$. Se $p \geq 2$ então para cada $\epsilon>0$, existe uma constante $C_{1}(\epsilon)$ positiva, tal que para cada $u \in D\left(-\Delta_{Z}\right)$,

$$
\|V u\|_{L^{2}} \leq\|V\|_{L^{p}}\left(\epsilon\left\|\Delta_{Z} u\right\|_{L^{2}}+C_{1}(\epsilon)\|u\|_{L^{2}}\right)
$$

Demonstração. Como $u \in H^{1}(\mathbb{R})$, então $\left(1+\xi^{2}\right)^{1 / 2} \widehat{u}(\xi) \in L^{2}(\mathbb{R})$, e como para $p \geq 2$,

$$
\int\left[\frac{1}{\left(1+\xi^{2}\right)^{1 / 2}}\right]^{p} d \xi \leq \int \frac{1}{1+\xi^{2}} d \xi=\pi,
$$

a desigualdade de Hölder estendida implica que

$$
\begin{aligned}
\|\widehat{u}\|_{L^{q}}=\left(\int|\widehat{u}(\xi)|^{q}\right)^{1 / q} d \xi & =\left(\int\left[\left(1+\xi^{2}\right)^{1 / 2}|\widehat{u}(\xi)|\left(1+\xi^{2}\right)^{-1 / 2}\right]^{q}\right)^{1 / q} d \xi \\
& \leq\left(\int\left(1+\xi^{2}\right)|\widehat{u}(\xi)|^{2}\right)^{1 / 2}\left(\int \frac{1}{\left(1+\xi^{2}\right)^{p / 2}} d \xi\right)^{1 / p} \\
& \leq \pi^{1 / p}\left(\|u\|_{L^{2}}+\left\|u^{\prime}\right\|_{L^{2}}\right)
\end{aligned}
$$

onde $\frac{1}{q}=\frac{1}{2}+\frac{1}{p} \leq 1$. É claro que $1 \leq q<2$. Logo, uma aplicação do Teorema de HausdorffYoung, implica que

$$
\|u\|_{L^{r}} \leq\|\widehat{u}\|_{L^{q}}
$$

$\operatorname{com} \frac{1}{r}+\frac{1}{q}=1$. A dependência da $r$ em relação da $p$, é expressada por

$$
r=\left\{\begin{array}{lll}
\frac{2 p}{p-2}, & \text { se } & p>2 \\
\infty, & \text { se } & p=2
\end{array}\right.
$$


Agora, da hipótese que $u \in D\left(\Delta_{Z}\right)$ decorre que

$$
\left\|u^{\prime}\right\|_{L^{2}} \leq\|u\|_{L^{2}}+\sqrt{|Z|}|u(0)|+\left\|\Delta_{Z} u\right\|_{L^{2}} .
$$

Pelo Lema de imersão de Sobolev, temos

$$
|u(0)| \leq \sqrt{\pi}\|u\|_{H^{1}} .
$$

Se substituímos $u(x)$ por $u(x / 4 Z \pi)$, obtemos

$$
|u(0)| \leq 2 \pi \sqrt{|Z|}\|u\|_{L^{2}}+\frac{1}{2 \sqrt{|Z|}}\left\|u^{\prime}\right\|_{L^{2}} .
$$

Logo, combinando as desigualdades (2.27) e (2.28), obtemos

$$
\frac{1}{2}\left\|u^{\prime}\right\|_{L^{2}} \leq(2 \pi|Z|+1)\|u\|_{L^{2}}+\left\|\Delta_{Z} u\right\|_{L^{2}}
$$

Assim, juntando toda a informação anterior, conseguimos

$$
\|u\|_{L^{r}} \leq C_{p}(Z)\left(\|u\|_{L^{2}}+\left\|\Delta_{Z} u\right\|_{L^{2}}\right) .
$$

No sucessivo, substituindo $u(x)$ por $u(\rho x), \rho>0$, na anterior desigualdade, conseguimos

$$
\|u\|_{L^{r}} \leq C_{p}(Z)\left(\rho^{\frac{1}{r}-\frac{3}{2}}\|u\|_{L^{2}}+\rho^{\frac{1}{2}+\frac{1}{r}}\left\|\Delta_{Z} u\right\|_{L^{2}}\right) .
$$

Logo, para $\epsilon>0$, temos do Teorema do Valor Intermediário que existe $\rho_{0}>0$ tal que $\rho_{0}^{\frac{1}{2}+\frac{1}{r}}=\epsilon$ e portanto, para $C_{1}(\epsilon)=\rho_{0}^{\frac{1}{r}-\frac{3}{2}}$, encontramos que

$$
\|u\|_{L^{r}} \leq C_{p}(Z)\left(C_{1}(\epsilon)\|u\|_{L^{2}}+\epsilon\left\|\Delta_{Z} u\right\|_{L^{2}}\right) .
$$

Finalmente, uma nova aplicação da desigualdade de Hölder estendida, implica que

$$
\|V u\|_{L^{2}} \leq\|V\|_{L^{p}}\|u\|_{L^{r}}
$$

Para obter o resultado desejado é só substituir (2.29) em esta última desigualdade.

Lema 2.7 Suponha que a função $V \in C\left(I ; L^{\tilde{p}}(\mathbb{R})\right)+C\left(I ; L^{\infty}(\mathbb{R})\right)$ e sua derivada respeito ao tempo $\frac{\partial V}{\partial t} \in L^{p_{1}, \alpha_{1}}(I)+L^{\infty, \beta}(I)$, onde $\tilde{p}=\max \{p, 2\}, p_{1}=\frac{2 p}{3}$ e $\alpha_{1}>\frac{4 p}{4 p-1}$, e defina as seguintes normas:

$$
\begin{gathered}
\|V\|_{\overline{\mathfrak{M}}}=\inf \left\{\left\|V_{1}\right\|_{L^{\tilde{p}, \infty}}+\left\|V_{2}\right\|_{L^{\infty, \infty}}: V=V_{1}+V_{2}\right\} \\
\|V\|_{\mathfrak{N}}=\inf \left\{\left\|W_{1}\right\|_{L^{p_{1}, \alpha_{1}}}+\left\|W_{2}\right\|_{L^{\infty, \beta},}: \frac{\partial V}{\partial t}=W_{1}+W_{2}\right\} .
\end{gathered}
$$

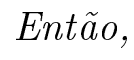

1. $V$ aplica $\mathfrak{J}(a)$ em $\mathfrak{J}^{*}(a)$ continuamente.

2. Para qualquer $\epsilon>0$, existe uma constante $C_{1}(\epsilon)$ positiva, tal que para $2 a<1$,

$$
\|V u\|_{\mathfrak{J}^{*}(a)} \leq\left(\epsilon\|V\|_{\overline{\mathfrak{M}}}+(2 a)^{\kappa}\left[\left\|V_{t}\right\|_{\mathfrak{N}}+\|V\|_{\overline{\mathfrak{M}}}\right]\right)\|u\|_{\mathfrak{J}(a, q)}+C_{1}(\epsilon)\|u\|_{L^{2, \infty}}\|V\|_{\overline{\mathfrak{M}}}
$$

para todo $u \in \mathfrak{J}(a)$, onde $\kappa=\min \left\{\frac{1}{\theta^{\prime}}-\frac{1}{\alpha_{1}}, \gamma\right\}$. 
Demonstração. Sejam $V=V_{1}+V_{2}$, onde $V_{1} \in C\left(I ; L^{\tilde{p}}(\mathbb{R})\right)$ e $V_{2} \in C\left(I ; L^{\infty}(\mathbb{R})\right)$, e $V_{t}=W_{1}+W_{2}$, onde $W_{1} \in L^{p_{1}, \alpha_{1}}(I)$ e $W_{2} \in L^{\infty, \beta}(I)$. Então, para $\tilde{p} \geq 2$, o Lema 2.6 mostra que

$$
\begin{aligned}
\|V u\|_{L^{2, \infty}} & \leq\left\|V_{1} u\right\|_{L^{2, \infty}}+\left\|V_{2} u\right\|_{L^{2, \infty}} \\
& \leq\left\|V_{1}\right\|_{L^{\tilde{p}, \infty}}\left(\epsilon\left\|\Delta_{Z} u\right\|_{L^{2, \infty}}+C_{1}(\epsilon)\|u\|_{L^{2, \infty}}\right)+\left\|V_{2}\right\|_{L^{\infty, \infty}}\|u\|_{L^{2, \infty}} \\
& \leq\left(\epsilon\left\|\Delta_{Z} u\right\|_{L^{2, \infty}}+C_{1}(\epsilon)\|u\|_{L^{2, \infty}}\right)\|V\|_{\overline{\mathfrak{M}}} .
\end{aligned}
$$

Por outro lado, a desigualdade de Hölder respeito à variável temporal, implica que

$$
\begin{aligned}
\left\|W_{2} u\right\|_{L^{2,1}} & \leq\left\|W_{2}\right\|_{L^{\infty, 1}}\|u\|_{L^{2, \infty}} \\
& \leq(2 a)^{1-\frac{1}{\beta}}\left\|W_{2}\right\|_{L^{\infty, \beta}}\|u\|_{\mathfrak{J}(a)} .
\end{aligned}
$$

Lembrando que $q=\frac{2 p}{2 p-1}$, então o seu expoente conjugado $q^{\prime}=\frac{2 p}{2 p+1}$ e $\theta=\frac{4 q}{q-2}$. Agora, sabemos que o espaço $H^{1}(\mathbb{R})$ está imerso continuamente em $L^{q_{1}}(\mathbb{R})$, com $q_{1}=\frac{2 p}{p-2}$. Logo, usando esta imeção com $u(t)$, temos

$$
\|u(t)\|_{L^{q_{1}}} \leq C_{p}\|u(t)\|_{H^{1}} .
$$

Portanto, usando a desigualdade de Hölder estendia na variável espacial, tomando $q_{1}, q^{\prime}$ como acima e $p_{1}=\frac{2 p}{3}$, e posteriormente aplicando a desigualdade de Hölder na variável temporal, com expoentes $\frac{\alpha_{1}}{\theta^{\prime}}$ e $\frac{\alpha_{1}}{\alpha_{1}-\theta^{\prime}}$, conseguimos

$$
\begin{aligned}
\left\|W_{1} u\right\|_{L^{q^{\prime}, \theta^{\prime}}} & \leq\left\|W_{1}\right\|_{L^{p_{1}, \theta^{\prime}}}\|u\|_{L^{q_{1}, \infty}} \\
& \leq(2 a)^{\frac{1}{\theta^{\prime}}-\frac{1}{\alpha_{1}}}\left\|W_{1}\right\|_{L^{p_{1}, \alpha_{1}}}\|u\|_{L^{q_{1}, \infty}},
\end{aligned}
$$

onde $\theta^{\prime}=\frac{4 p}{4 p-1}$. Assim, da desigualdade (2.34), das relações (2.12) e (2.13), e da definição da norma sobre espaço $\mathfrak{J}(a, q)$, temos

$$
\begin{aligned}
\left\|W_{1} u\right\|_{L^{q^{\prime}, \theta^{\prime}}} & \leq C_{p}(Z)(2 a)^{\frac{1}{\theta^{\prime}}-\frac{1}{\alpha_{1}}}\left\|W_{1}\right\|_{L^{p_{1}, \alpha_{1}}}\left(\|u\|_{L^{2, \infty}}+\left\|\Delta_{Z} u\right\|_{L^{2, \infty}}\right) \\
& \leq C_{p}(Z)(2 a)^{\frac{1}{\theta^{\prime}}-\frac{1}{\alpha_{1}}}\left\|W_{1}\right\|_{L^{p_{1}, \alpha_{1}}} \sup _{t}\|u\|_{Z} \\
& \leq C_{p}(Z)(2 a)^{\frac{1}{\theta^{\prime}}-\frac{1}{\alpha_{1}}}\left\|W_{1}\right\|_{L^{p_{1}, \alpha_{1}}}\|u\|_{\mathfrak{J}(a)} .
\end{aligned}
$$

Por outro lado, a desigualdade (2.24) mostra que

$$
\left\|u_{t} V\right\|_{\mathfrak{X}^{*}(a, q)} \leq(2 a)^{\gamma}\|V\|_{\mathfrak{M}}\left\|u_{t}\right\|_{\mathfrak{X}(a, q)} .
$$

Por conseguinte, usando toda a informação anterior obtemos que

$$
\begin{aligned}
\|V u\|_{\mathfrak{J}^{*}(a, q)} & =\|V u\|_{L^{2, \infty}}+\left\|\frac{d}{d t}(V u)\right\|_{\mathfrak{X}^{*}(a, q)} \\
\leq & \left(\epsilon\|u\|_{\mathfrak{J}(a, q)}+C_{1}(\epsilon)\|u\|_{L^{2, \infty}}\right)\|V\|_{\overline{\mathfrak{M}}}+\left\|V_{t} u\right\|_{\mathfrak{X}^{*}(a, q)}+\left\|V u_{t}\right\|_{\mathfrak{X}^{*}(a, q)} \\
\leq & \left(\epsilon\|u\|_{\mathfrak{J}(a, q)}+C_{1}(\epsilon)\|u\|_{L^{2, \infty}}\right)\|V\|_{\overline{\mathfrak{M}}}+\left\|W_{2} u\right\|_{L^{2,1}}+\left\|W_{1} u\right\|_{L^{q^{\prime}, \theta^{\prime}}} \\
& \quad+(2 a)^{\gamma}\|V\|_{\mathfrak{M}}\left\|u_{t}\right\|_{\mathfrak{X}(a, q)} \\
\leq & \left(\epsilon\|u\|_{\mathfrak{J}(a, q)}+C_{1}(\epsilon)\|u\|_{L^{2, \infty}}\right)\|V\|_{\overline{\mathfrak{M}}}+(2 a)^{\gamma}\|V\|_{\overline{\mathfrak{M}}}\left\|u_{t}\right\|_{\mathfrak{X}(a, q)} \\
& \quad+(2 a)^{1-\frac{1}{\beta}}\left\|W_{2}\right\|_{L^{\infty, \beta}}\|u\|_{\mathfrak{J}(a)}+(2 a)^{\frac{1}{\theta^{\prime}}-\frac{1}{\alpha_{1}}}\left\|W_{1}\right\|_{L^{p_{1}, \alpha_{1}}}\|u\|_{\mathfrak{\mathfrak { J }}(a)} \\
\leq & \left(\epsilon\|V\|_{\overline{\mathfrak{M}}}+(2 a)^{\kappa}\left\|V_{t}\right\|_{\mathfrak{N}}\right)\|u\|_{\mathfrak{J}(a, q)}+\left(C_{1}(\epsilon)\|u\|_{L^{2, \infty}}+(2 a)^{\kappa}\|u\|_{\mathfrak{J}(a, q)}\right)\|V\|_{\overline{\mathfrak{M}}},
\end{aligned}
$$

onde também levamos em consideração que $\|V\|_{\mathfrak{M}} \leq\|V\|_{\overline{\mathfrak{M}}}$ e $\kappa=\min \left\{\gamma, \frac{1}{\theta^{\prime}}-\frac{1}{\alpha_{1}}, 1-\frac{1}{\beta}\right\}$. Essa desigualdade mostra que $V$ aplica o espaço $\mathfrak{J}(a)$ continuamente no espaço $\mathfrak{J}^{*}(a)$. 


\subsection{Existência local e global em $L^{2}(\mathbb{R})$ e $D\left(-\Delta_{Z}\right)$}

Nesta seção mostraremos a existência e unicidade da soluções do problema (2.1) com dado inicial em $L^{2}(\mathbb{R})$ e em $D\left(-\Delta_{Z}\right)$. Iniciamos com o resultado básico de que uma solução da equação integral (2.2) é solução do problema (2.1).

Lema 2.8 Suponha que $u \in \mathfrak{X}(T, q)$ satisfaz

$$
u(t)=U_{Z}(t) u_{0}+i S_{Z} V u(t), \quad u_{0} \in D\left(-\Delta_{Z}\right),
$$

e $V u \in \mathfrak{J}^{*}(T, q)$. Então $u \in \mathfrak{J}(T, q)$, satisfaz a equação diferencial e

$$
\frac{d u}{d t}=i\left(\Delta_{Z}+V(x, t)\right) u
$$

$e$

$$
\frac{d}{d t} S_{Z} g(t)=U_{Z}(t) g(0)+S_{Z} \dot{g}(t), \quad \text { com } \quad g=V u .
$$

Demonstração. Já que para cada $u_{0} \in D\left(-\Delta_{Z}\right)$, temos $\Delta_{Z}\left[U_{Z}(t) u_{0}\right]=U_{Z}(t) \Delta_{Z} u_{0}$, então $U_{Z}(t)$ representa uma isometria sobre $\left(D\left(-\Delta_{Z}\right),\|\cdot\|_{Z}\right)$,

$$
\begin{aligned}
\left\|U_{Z}(t) u_{0}\right\|_{Z} & =\left\|\left(-\Delta_{Z}+m\right) U_{Z}(t) u_{0}\right\|_{L^{2}} \\
& =\left\|\left(-\Delta_{Z}+m\right) u_{0}\right\|_{L^{2}} \\
& =\left\|u_{0}\right\|_{Z} .
\end{aligned}
$$

Por outro lado, do Lema 2.1 e a desigualdade (2.13), obtemos para $Z>0$ que

$$
\begin{aligned}
\left\|\frac{d}{d t} U_{Z}(t) u_{0}\right\|_{\mathfrak{X}(T, q)} & =\left\|U_{Z}(t) \Delta_{Z} u_{0}\right\|_{\mathfrak{X}(T, q)} \\
& \leq C(Z)\left\|\Delta_{Z} u_{0}\right\|_{L^{2}} \\
& \leq C(Z)\left\|u_{0}\right\|_{Z}
\end{aligned}
$$

e para $Z<0$, conseguimos

$$
\begin{aligned}
\left\|\frac{d}{d t} U_{Z}(t) u_{0}\right\|_{\mathfrak{X}(T, q)} & =\left\|U_{Z}(t) \Delta_{Z} u_{0}\right\|_{\mathfrak{X}(T, q)} \\
& \leq C(Z)\left((2 T)^{1 / \theta}+1\right)\left\|\Delta_{Z} u_{0}\right\|_{L^{2}} \\
& \leq C(Z)\left((2 T)^{1 / \theta}+1\right)\left\|u_{0}\right\|_{Z} .
\end{aligned}
$$

Portanto, para $Z>0$, de (2.41) e (2.43)

$$
\begin{aligned}
\left\|U_{Z}(t) u_{0}\right\|_{\mathfrak{J}(T, q)} & =\sup _{t}\left\|U_{Z}(t) u_{0}\right\|_{Z}+\left\|\frac{d}{d t} U_{Z}(t) u_{0}\right\|_{\mathfrak{X}(T, q)} \\
& \leq c(Z)\left\|u_{0}\right\|_{Z},
\end{aligned}
$$

e para $Z<0$, de $(2.41)$ e $(2.44)$

$$
\left\|U_{Z}(t) u_{0}\right\|_{\mathfrak{J}(T, q)} \leq C(Z)\left((2 T)^{1 / \theta}+1\right)\left\|u_{0}\right\|_{Z}
$$

Logo, como $V u \in \mathfrak{J}^{*}(T, q)$, os Lemas 2.4 e 2.5, e a desigualdade (2.46) resulta para $Z>0$, que

$$
\begin{aligned}
\|u\|_{\mathfrak{J}(T, q)} & \leq\left\|U_{Z}(t) u_{0}\right\|_{\mathfrak{J}(T, q)}+\left\|S_{Z}(t) V u\right\|_{\mathfrak{J}(T, q)} \\
& \leq C(Z)\left(\left\|u_{0}\right\|_{Z}+(T+1)(2 T)^{\gamma}\|V\|_{\mathfrak{M}}\|u\|_{\mathfrak{J}^{*}(T, q)}\right) .
\end{aligned}
$$


Para $Z<0$, do Lema 2.4 e (2.46)

$$
\|u\|_{\mathfrak{J}(T, q)} \leq C(Z)\left(T+(2 T)^{1 / \theta}+1\right)\left(\left\|u_{0}\right\|_{Z}+(2 T)^{\gamma}\|V\|_{\mathfrak{M}}\|u\|_{\mathfrak{J}^{*}(T, q)}\right) .
$$

Portanto, $S_{Z}(V u) \in \mathfrak{X}(T, q) \cap \mathfrak{J}(T, q)$, assim

$$
Q_{Z} u(t) \equiv S_{Z} V u(t)=\int_{0}^{t} U_{Z}(t-s) V(s) u(s) d s,
$$

é contínua em $D\left(-\Delta_{Z}\right)$ e a sua derivada $\frac{d Q_{Z} u}{d t}$ é uma função contínua de $[0, T]$ em $L^{2}(\mathbb{R})$. Também, como $V u \in C\left([0, T]: L^{2}(\mathbb{R})\right)$, então

$$
\int_{0}^{T}\|(V u)(t)\|_{L^{2}} d t \leq T\|V u\|_{L^{2, \infty}} .
$$

Por conseguinte,

$$
\frac{d Q_{Z} u(t)}{d t}=\frac{d}{d t} S_{Z}(V u)(t)=i \Delta_{Z} Q_{Z} u(t)+V(t) u(t)
$$

Derivando com respeito a $t$ a equação integral (2.38), obtemos

$$
\begin{aligned}
u_{t} & =i \Delta_{Z} U_{Z}(t) u_{0}+i \frac{d}{d s} Q_{Z} u(t) \\
& =i \Delta_{Z} U_{Z}(t) u_{0}+i \Delta_{Z} Q_{Z} u(t)+i V(t) u(t) \\
& =i \Delta_{Z}\left(U_{Z}(t) u_{0}+Q_{Z} u(t)\right)+i V(t) u(t) \\
& =i\left[\Delta_{Z}+V(t)\right] u(t) .
\end{aligned}
$$

Finalmente, das propriedades de grupo unitário encontramos que

$$
\begin{aligned}
g(t)-U_{Z}(t) g(0) & =\int_{0}^{t} \frac{d}{d s} U_{Z}(t-s) g(s) d s \\
& =\int_{0}^{t}\left(-i \Delta_{Z}\right) U_{Z}(t-s) g(s) d s+\int_{0}^{t} U_{Z}(t-s) g_{s}(s) d s \\
& =-i \Delta_{Z} S_{Z} g(t)+S_{Z} g_{s}(t),
\end{aligned}
$$

logo, usando a derivada $\frac{d Q_{Z} u}{d t}$ calculada conseguimos imediatamente (2.40).

O seguinte lema nos diz que uma solução $u(t)$ de equação integral (2.2) está proxima da solução $u_{\epsilon}(t)$ da equação integral

$$
u_{\epsilon}(t)=U_{Z}(t) u_{0 \epsilon}+i S_{Z} V_{\epsilon} u_{\epsilon}(t),
$$

na norma $\mathfrak{X}(T, q)$, sempre que os potenciais $V$ e $V_{\epsilon}$ e os dados iniciais $u_{0}$ e $u_{0 \epsilon}$ estejam próximos. Esse resultado será muito útil para mostrar a conservação da norma $L^{2}(\mathbb{R})$ da solução $u(t)$.

Lema 2.9 Suponha que para $2 T<1, p \geq 1, \alpha \geq 1, \beta>1$, com $0 \leq \frac{1}{\alpha}<1-\frac{1}{2 p}$, $V_{\epsilon}$, $V \in L^{p, \alpha}(I)+L^{\infty, \beta}(I), \operatorname{com} q=\frac{2 p}{2 p-1}(\epsilon>0)$,

$$
\lim _{\epsilon \rightarrow 0}\left\|V_{\epsilon}-V\right\|_{\mathfrak{M}}=0 .
$$


Suponha também que

$$
\lim _{\epsilon \rightarrow 0}\left\|u_{0 \epsilon}-u_{0}\right\|_{L^{2}}=0 .
$$

Sejam $u \in \mathfrak{X}(T, q)$ e $u_{\epsilon} \in \mathfrak{X}(T, q)$ as soluções de (2.38) e (2.49), respectivamente. Então

$$
\lim _{\epsilon \rightarrow 0}\left\|u_{\epsilon}-u\right\|_{\mathfrak{X}(T, q)}=0 .
$$

Demonstração. Denotamos $Q_{Z_{\epsilon}}=S_{Z} V_{\epsilon}$. Agora, consideremos para $0<a \leq T$, o espaço

$$
\mathfrak{X}(a)=\mathfrak{X}(a, q), \quad q=\frac{2 p}{p-1} .
$$

Segue-se dos Lemas 2.3 e 2.5, que o operador integral

$$
\left(Q_{Z} u\right)(t)=\int_{0}^{t} U_{Z}(t-s) V(s) u(s) d s
$$

é limitada sobre $\mathfrak{X}(a)$, pois para $Z>0$

$$
\begin{aligned}
\left\|Q_{Z} u\right\|_{\mathfrak{X}(a)} & =\left\|S_{Z} V u\right\|_{\mathfrak{X}(a)} \leq c\|V u\|_{\mathfrak{X}^{*}(a)} \\
& \leq(2 a)^{\gamma} c\|V\|_{\mathfrak{M}}\|u\|_{\mathfrak{X}(a)}, \quad 2 a<1, \quad \gamma>0,
\end{aligned}
$$

e para $Z<0$,

$$
\begin{aligned}
\left\|Q_{Z} u\right\|_{\mathfrak{X}(a)} & \leq c(2 a)^{\gamma}\left((2 a)^{\frac{1}{\theta}}+1\right)\|V\|_{\mathfrak{M}}\|u\|_{\mathfrak{X}^{*}(a, l)} \\
& \leq(2 a)^{\gamma} c\|V\|_{\mathfrak{M}}\|u\|_{\mathfrak{X}(a)}, \quad 2 a<1, \quad \gamma>0 .
\end{aligned}
$$

Portanto, se $a$ é suficientemente pequeno tal que

$$
c(2 a)^{\gamma}\|V\|_{\mathfrak{M}} \leq \frac{1}{2}
$$

temos que o operador $Q_{Z}$ é uma contração sobre $\mathfrak{X}(a)$ :

$$
\left\|Q_{Z} u\right\|_{\mathfrak{X}(a)} \leq \frac{1}{2}\|u\|_{\mathfrak{X}(a)} .
$$

Observemos que para esse mesmo $a$ o operador $Q_{Z_{\epsilon}}$ também é uma contração sobre $\mathfrak{X}(a)$.

Por outro lado, para $Z>0$, a desigualdade (2.3), implica que

$$
\left\|U_{Z}(\cdot)\left(u_{0 \epsilon}-u_{0}\right)\right\|_{\mathfrak{X}(T)} \leq c\left\|u_{0 \epsilon}-u_{0}\right\|_{L^{2}},
$$

e para $Z<0$, a desigualdade (2.4) mostra que

$$
\left\|U_{Z}(\cdot)\left(u_{0 \epsilon}-u_{0}\right)\right\|_{\mathfrak{X}(T)} \leq C\left((2 T)^{\frac{1}{\theta}}+1\right)\left\|u_{0 \epsilon}-u_{0}\right\|_{L^{2}}
$$

Logo, para $Z \in \mathbb{R}$, as anteriores duas desigualdade e a hipótese (2.51), temos

$$
\lim _{\epsilon \rightarrow 0}\left\|U_{Z}(\cdot)\left(u_{0 \epsilon}-u_{0}\right)\right\|_{\mathfrak{X}(T)}=0 .
$$

Também, da hipótese (2.50) e (2.53), conseguimos

$$
\lim _{\epsilon \rightarrow 0}\left\|Q_{Z}-Q_{Z_{\epsilon}}\right\|_{\mathfrak{B}(\mathfrak{X}(T))}=0,
$$

onde $\|\cdot\|_{\mathfrak{B}(\mathfrak{X}(T))}$ é a norma definida sobre o espaço, $\mathfrak{B}(\mathfrak{X}(T))$, dos operadores limitados sobre $\mathfrak{X}(T)$. Assim, combinando estes dois últimos fatos, obtemos (2.52).

O seguinte resultado estabelece a boa colocação em $L^{2}(\mathbb{R})$ da equação integral (2.2). Além disso, usamos o Lema 2.9 para mostrar que a norma $L^{2}$ da solução é conservada. 
Teorema 2.10 (i) Suponha que para $p \geq 1$, $\alpha \geq 1, \beta>1$, com $0 \leq \frac{1}{\alpha}<1-\frac{1}{2 p}$, $V \in L^{p, \alpha}+L^{\infty, \beta}$, com $q=\frac{2 p}{p-1}$. Então, para cada $u_{0} \in L^{2}(\mathbb{R})$ a equação (2.38) tem uma única solução $u \in C\left([0, T]: L^{2}(\mathbb{R})\right) \cap L^{q, \theta}(I), \theta=\theta(q)=4 p$.

(ii) Sejam $V \in C^{1}\left([0, T]: L^{\infty}(\mathbb{R})\right)$ e $u_{0} \in D\left(-\Delta_{Z}\right)$. Então a solução $u \in \mathfrak{X}(T)$ da equação integral (2.38) pertence a $\mathfrak{J}(T)$ e satisfaz (2.1).

(iii) $\|u(t)\|_{L^{2}}=\left\|u_{0}\right\|_{L^{2}}$, para todo $t \in[0, T]$.

Demonstração. (i) No Lema 2.9 mostramos que para $0 \leq a<1 / 2$ tal que

$$
c(2 a)^{\gamma}\|V\|_{\mathfrak{M}} \leq \frac{1}{2},
$$

o operador $Q_{Z}: \mathfrak{X}(a) \rightarrow \mathfrak{X}(a)$, satisfaz $\left\|Q_{Z}\right\|_{\mathfrak{B}(\mathfrak{X}(a))} \leq 1 / 2$. Então, o operador $I-i Q_{Z}$ é invertível e como pela desigualdade $(2.3), f(t)=U_{Z}(t) u_{0} \in \mathfrak{X}(a)$, para qualquer $u_{0} \in L^{2}(\mathbb{R})$, segue-se que a equação integral

$$
u(t)=u_{0}(t)+i\left(Q_{Z} u\right)(t)
$$

tem única solução $u(t)=\left(I-i Q_{Z}\right)^{-1} f(t) \in \mathfrak{X}(a)$.

(ii) Defina para $h \neq 0$ pequeno,

$$
v_{h}(t)=\frac{u(t+h)-u(t)}{h}
$$

Então $v_{h}(t)$ satisfaz a equação,

$$
v_{h}(t)=f_{h}(t)+i\left(Q_{Z} v_{h}\right)(t)
$$

onde

$$
\begin{aligned}
f_{h}(t) & =U_{Z}(t) \frac{\left(U_{Z}(h)-I\right)}{h} u_{0}+i \frac{U_{Z}(t+h)}{h} \int_{0}^{h} U_{Z}(-s) V(s) u(s) d s \\
& +i \int_{0}^{h} U_{Z}(t-s) \frac{(V(s+h)-V(s))}{h} u(s+h) d s .
\end{aligned}
$$

Como $V \in C^{1}\left([0, T]: L^{\infty}(\mathbb{R})\right)$, então $V \in L^{\infty, \beta}(I)$ e assim satisfaz as hipóteses dadas no item (i). Logo, existe $a>0$ suficientemente pequeno para o qual existe uma solução $u \in \mathfrak{X}(a)$ de (2.38).

A continuação vamos mostrar que $V u \in \mathfrak{J}^{*}(a)=\mathfrak{J}^{*}(a, q)$. Primeiro, observemos que $V_{t} \in$ $L^{\infty, \beta}(I), \operatorname{assim} V u, V_{t} u \in \mathfrak{X}^{*}(a)$ e portanto do Lema 2.3, segue-se que $S_{Z} V u, S_{Z} V_{t} u \in \mathfrak{X}(a)$. Só resta mostrar que $u_{t} \in \mathfrak{X}(a)$. Já que $u_{0} \in D\left(-\Delta_{Z}\right)$ e $u \in \mathfrak{X}(a)$ o Lema 2.1 implica que

$$
f_{h}(t) \rightarrow f(t)=i\left[U_{Z}(t)\left(\Delta_{Z}+V(0)\right) u_{0}+S_{Z} V_{t} u(t)\right], \quad h \rightarrow 0
$$

em $\mathfrak{X}_{l o c}(a)$. Portanto, pela propriedade de contração do operador $Q_{Z}=S_{Z} V$ em $\mathfrak{X}(a)$, temos

$$
v_{h}=\left(I-i Q_{Z}\right)^{-1} f_{h} \rightarrow\left(I-i Q_{Z}\right)^{-1} f, \quad h \rightarrow 0
$$

em $\mathfrak{X}_{l o c}(a)$. Isto mostra que

$$
\frac{d u}{d t}=\left(I-i Q_{Z}\right)^{-1} f
$$


em $\mathfrak{X}(a)$ e

$$
\frac{d u}{d t}=i\left[U_{Z}(t)\left(\Delta_{Z}+V(0)\right) u_{0}+S_{Z} V_{t} u(t)+S_{Z} V u_{t}(t)\right]
$$

Consequentemente, $V u_{t} \in \mathfrak{X}^{*}(a)$, donde $V u \in \mathfrak{J}^{*}(a)$. Logo o Lema 2.8 implica o resultado desejado.

(iii) Inicialmente aproximamos $V=V_{1}+V_{2} \in L^{p, \alpha}(I)+L^{\infty, \beta}(I)$ por $V_{\epsilon} \in C^{1}\left([0, T]: L^{\infty}(\mathbb{R})\right)$. Para isso, tomemos $\tau \in C_{0}^{\infty}(\mathbb{R})$ tal que $\tau \geq 0$ e $\int \tau(x) d x=1$, e definamos

$$
V_{\epsilon}(x, t)=\iint V_{1}(x+\epsilon y, t+\epsilon s) \tau(x) \tau(t) d x d s+\int V_{2}(x, t+\epsilon s) d s,
$$

onde, estendemos $V_{j}(x, t)$ fora da região $I \times \mathbb{R} \operatorname{como} V_{i}(x, t) \equiv 0, \mathrm{j}=1,2$. Então,

$$
V_{\epsilon} \in C^{1}\left([0, T]: L^{\infty}(\mathbb{R})\right), \quad\left\|V_{\epsilon}-V\right\|_{\mathfrak{M}} \longrightarrow 0, \text { quando } \epsilon \rightarrow 0 .
$$

Como o espaço $D\left(-\Delta_{Z}\right)$ é denso em $L^{2}(\mathbb{R})$, para $u_{0} \in L^{2}(\mathbb{R})$ e cada $\epsilon>0$, existe uma função $u_{0 \epsilon} \in D\left(-\Delta_{Z}\right)$, tal que

$$
\left\|u_{0}-u_{0 \epsilon}\right\|_{L^{2}}<\epsilon
$$

então, pela parte (i), existe uma única solução $u_{\epsilon} \in \mathfrak{X}(T)$ que satisfaz

$$
\left\{\begin{array}{l}
\partial_{t} u_{\epsilon}=i\left(\Delta_{Z} u_{\epsilon}+V_{\epsilon}(x, t) u_{\epsilon}\right), \quad x \in \mathbb{R}, \quad t \in \mathbb{R} \\
u(x, 0)=u_{0 \epsilon},
\end{array}\right.
$$

Logo da parte (ii) obtemos que $u_{\epsilon} \in \mathfrak{J}(T)$. Portanto o Lema 2.9 implica que,

$$
\lim _{\epsilon \rightarrow 0}\left\|u_{\epsilon}-u\right\|_{\mathfrak{X}(T)}=0 .
$$

Agora como- $\Delta_{Z}+V_{\epsilon}(t)$ é autoadjunto com domínio $D\left(-\Delta_{Z}\right)$, é imediato ver de $(2.55)$ que $\frac{d}{d t}\left\|u_{\epsilon}(t)\right\|_{L^{2}}=0$ e por conseguinte, $\left\|u_{\epsilon}(t)\right\|_{L^{2}}=\left\|u_{0 \epsilon}\right\|_{L^{2}}$. Então, fazendo $\epsilon \rightarrow 0$, obtemos a propriedade $\|u(t)\|_{L^{2}}=\left\|u_{0}\right\|_{L^{2}}$.

Finalizamos este capítulo com mais um resultado sobre o comportamento das soluções de (2.1).

Teorema 2.11 Sejam $V \in C^{1}\left([0, \infty]: L^{\tilde{p}}(\mathbb{R})\right)+C^{1}\left([0, \infty]: L^{\infty}(\mathbb{R})\right)$ e $u_{0} \in D\left(-\Delta_{Z}\right)$. Então, para algum $T^{\prime}>0$, a solução $u \in \mathfrak{X}\left(T^{\prime}\right)$ de (2.1) correspondente a $u_{0}$, satisfaz que $u \in \mathfrak{J}\left(T^{\prime}\right) e$

$$
\partial_{t} u(x, t)=i\left(\Delta_{Z}+V(x, t)\right) u(x, t) .
$$

A solução pode ser estendida globalmente, isto é, para cada $T>0$, a solução $u \in \mathfrak{J}(T)$.

Demonstração. Da hipótese sobre a função $V$, podemos escrever esta como $V=V_{1}+V_{2}$, com $V_{1} \in C^{1}\left([0, T]: L^{\tilde{p}}(\mathbb{R})\right)$ e $V_{2} \in C^{1}\left([0, T]: L^{\infty}(\mathbb{R})\right)$. Tomemos uma função não negativa $\rho \in C_{0}^{\infty}(\mathbb{R}) \operatorname{com} \int_{\mathbb{R}} \rho(x) d x=1$, e para $\epsilon>0$ defina,

$$
\begin{gathered}
V_{1, \epsilon}(x, t)=\int V_{1}(x+\epsilon y, t) \rho(y) d y, \quad t \in[0, T], \\
V_{\epsilon}(x, t)=V_{1, \epsilon}(x, t)+V_{2}(x, t) .
\end{gathered}
$$

É fácil ver então que $V_{\epsilon} \in C^{1}\left([0, T]: L^{\infty}(\mathbb{R})\right)$ e

$$
\lim _{\epsilon \rightarrow 0}\left(\left\|V_{\epsilon}-V\right\|_{\overline{\mathfrak{M}}}+\left\|\partial_{t} V_{\epsilon}-\partial_{t} V\right\|_{\mathfrak{N}}\right)=0
$$


Portanto, pelo Lema 2.9 e o item (ii) do Teorema 2.10, a solução $u_{\epsilon}$ da equação integral,

$$
u_{\epsilon}(t)=U_{Z}(t) u_{0}+i S_{Z} V_{\epsilon} u_{\epsilon}(t), \quad u_{0} \in D\left(-\Delta_{Z}\right)
$$

pertence a $\mathfrak{J}(T)$ e satisfaz

$$
\begin{aligned}
\left\|u_{\epsilon}(t)\right\|_{L^{2}} & =\left\|u_{0}\right\|_{L^{2}} \\
\left\|u_{\epsilon}-u\right\|_{\mathfrak{X}(T)} & \rightarrow 0, \quad \text { quando } \quad \epsilon \rightarrow 0 .
\end{aligned}
$$

Então, aplicando os Lemas 2.4 e 2.7 a (2.59), e usando a lei de conservação (2.60), temos que para qualquer $\delta>0$ e $Z>0$,

$$
\begin{aligned}
\left\|u_{\epsilon}\right\|_{\mathfrak{J}(T)} \leq & \left\|U_{Z}(t) u_{0}\right\|_{\mathfrak{J}(T)}+\left\|S_{Z} V_{\epsilon} u_{\epsilon}(t)\right\|_{\mathfrak{J}(T)} \\
\leq & C(Z)\left(\left\|u_{0}\right\|_{Z}+(1+T)\left\|V_{\epsilon} u_{\epsilon}\right\|_{\mathfrak{J}^{*}(a, l)}\right) \\
\leq & C(Z)(1+T)\left[\left\|u_{0}\right\|_{Z}+\left(\delta\left\|V_{\epsilon}\right\|_{\overline{\mathfrak{M}}}+(T)^{\kappa} \mid\left(\left\|\partial_{t} V_{\epsilon}\right\|_{\mathfrak{N}}+\left\|V_{\epsilon}\right\|_{\overline{\mathfrak{M}}}\right)\right)\left\|u_{\epsilon}\right\|_{\mathfrak{J}(T)}\right. \\
& \left.\quad+C_{1}(\delta)\left\|u_{\epsilon}\right\|_{L^{2, \infty}}\left\|V_{\epsilon}\right\|_{\overline{\mathfrak{M}}}\right] \\
& \quad+C(Z)(1+T)\left[\left\|u_{0}\right\|_{Z}+\left(\delta\left\|V_{\epsilon}\right\|_{\overline{\mathfrak{M}}}+(T)^{\kappa}\left(\left\|\partial_{t} V_{\epsilon}\right\|_{\mathfrak{N}}+\left\|V_{\epsilon}\right\|_{\overline{\mathfrak{M}}}\right)\right)\left\|u_{\epsilon}\right\|_{\mathfrak{J}(T)}\right. \\
& \left.\quad C_{1}(\delta)\left\|u_{0}\right\|_{L^{2}}\left\|V_{\epsilon}\right\|_{\overline{\mathfrak{M}}}\right],
\end{aligned}
$$

onde $C(Z)$ e $C_{1}(\delta)$ são constantes independentes de $T<1$. Portanto, escolhendo $0<\delta<1$ e $0<T<1$ pequenos tal que

$$
\begin{aligned}
\sup _{\epsilon>0} C(Z)(1+T) & \left(\delta\left\|V_{\epsilon}\right\|_{\overline{\mathfrak{M}}}+(T)^{\kappa}\left[\left\|\partial_{t} V_{\epsilon}\right\|_{\mathfrak{N}}+\left\|V_{\epsilon}\right\|_{\overline{\mathfrak{M}}}\right]\right) \\
& \leq C(Z)(1+T)\left(\delta\|V\|_{\overline{\mathfrak{M}}}+(T)^{\kappa}\left[\left\|\partial_{t} V\right\|_{\mathfrak{N}}+\|V\|_{\overline{\mathfrak{M}}}\right]\right) \\
& <\frac{1}{2}
\end{aligned}
$$

vemos que para $0<\epsilon<1$,

$$
\left\|u_{\epsilon}\right\|_{\mathfrak{J}(T)} \leq C(Z)\left(\left\|u_{0}\right\|_{Z}+C_{1}(\delta)\|V\|_{\overline{\mathfrak{M}}}\left\|u_{0}\right\|_{L^{2}}\right)
$$

Estimando similarmente como em (2.62), temos

$$
\begin{aligned}
\left\|u_{\epsilon_{1}}-u_{\epsilon_{2}}\right\|_{\mathfrak{J}(T)} \leq C(Z) & (1+T)\left[\left(\delta\left\|V_{\epsilon_{2}}-V_{\epsilon_{1}}\right\|_{\overline{\mathfrak{M}}}+T^{\kappa}\left(\left\|\partial_{t} V_{\epsilon_{2}}-\partial_{t} V_{\epsilon_{1}}\right\|_{\mathfrak{N}}\right.\right.\right. \\
& \left.\left.\left.+\left\|V_{\epsilon_{2}}-V_{\epsilon_{1}}\right\|_{\overline{\mathfrak{M}}}\right)\right)\left\|u_{\epsilon_{2}}\right\|_{\mathfrak{J}(T)}+C_{1}(\delta)\left\|u_{0}\right\|_{L^{2}}\left\|V_{\epsilon_{2}}-V_{\epsilon_{1}}\right\|_{\overline{\mathfrak{M}}}\right] \\
+C(Z) & (1+T)\left[\left(\delta\left\|V_{\epsilon_{1}}\right\|_{\overline{\mathfrak{M}}}+T^{\kappa}\left(\left\|\partial_{t} V_{\epsilon_{1}}\right\|_{\mathfrak{N}}+\left\|V_{\epsilon_{1}}\right\|_{\overline{\mathfrak{M}}}\right)\right)\left\|u_{\epsilon_{2}}-u_{\epsilon_{1}}\right\|_{\mathfrak{J}(T)}\right. \\
& \left.+C_{1}(\delta)\left\|u_{\epsilon_{2}}-u_{\epsilon_{1}}\right\|_{L^{2, \infty}}\left\|V_{\epsilon_{1}}\right\|_{\overline{\mathfrak{M}}}\right] .
\end{aligned}
$$

Assim, escolhendo $\delta$ e $T>0$ como em (2.63) e usando as relações (2.58), (2.61) e (2.64), vemos que $u_{\epsilon} \rightarrow u$ em $\mathfrak{J}(T)$ quando $\epsilon \rightarrow 0$. Isto mostra que $u \in \mathfrak{J}(T)$. Já que $V u \in \mathfrak{J}^{*}(T)$, então o Lema 2.8 implica (2.57). Por técnicas padrões, como é feito em [5], sabemos que existe um tempo maximal $T^{*}$ de existência para a solução, 
Agora usaremos a lei de conservação para mostrar que as soluções são globais no tempo. Suponha que $T^{*}<\infty$. Devido a que

$$
\begin{aligned}
i\left(-\Delta_{Z}+m\right) \int_{0}^{t} e^{i \Delta_{Z}(t-s)} V(s) u(s) d s & =i m \int_{0}^{t} e^{i \Delta_{Z}(t-s)} V(s) u(s) d s+e^{i t \Delta_{Z}} V(0) u(0) \\
& -V(t) u(t)+\int_{0}^{t} e^{i \Delta_{Z}(t-s)} V(s) u_{s}(s) d s \\
& +\int_{0}^{t} e^{i \Delta_{Z}(t-s)} V_{s}(s) u(s) d s
\end{aligned}
$$

temos

$$
\begin{aligned}
\|u(t)\|_{Z} & \leq\left\|U_{Z}(t) u_{0}\right\|_{Z}+\left\|\int_{0}^{t} e^{i \Delta_{Z}(t-s)} V(s) u(s) d s\right\|_{Z} d s \\
& \leq\left\|u_{0}\right\|_{L^{2}}+m \int_{0}^{t}\|V(s) u(s)\|_{L^{2}} d s+\left\|V(0) u_{0}\right\|_{L^{2}}+\|V(s) u(s)\|_{L^{2}} \\
& +\int_{0}^{t}\left\|V(s) u_{s}(s)\right\|_{L^{2}} d s+\int_{0}^{t}\left\|V_{s}(s) u(s)\right\|_{L^{2}} d s .
\end{aligned}
$$

Usando a preservação da norma $L^{2}(\mathbb{R})$ para a solução $u$ e usando a equação diferencial em (2.1), conseguimos para qualquer $t<T^{*}$

$$
\begin{aligned}
\|u(t)\|_{Z} & \leq\left\|u_{0}\right\|_{L^{2}}+\left(2\|V\|_{L^{\infty, \infty}}+t\left(m\|V\|_{L^{\infty, \infty}}+\left\|V_{s}\right\|_{L^{\infty}, \infty}\right)\right)\left\|u_{0}\right\|_{L^{2}} \\
& +\|V\|_{L^{\infty}, \infty} \int_{0}^{t}\left\|\Delta_{Z} u(s)\right\|_{L^{2}} d s+\|V\|_{L^{\infty, \infty}} \int_{0}^{t}\|V(s) u(s)\|_{L^{2}} d s \\
& \leq\left\|u_{0}\right\|_{L^{2}}+\left(2\|V\|_{L^{\infty, \infty}}+t\left(m\|V\|_{L^{\infty, \infty}}+\left\|V_{s}\right\|_{L^{\infty, \infty}}+\|V\|_{L^{\infty}, \infty}^{2}\right)\right)\left\|u_{0}\right\|_{L^{2}} \\
& +\|V\|_{L^{\infty, \infty}} \int_{0}^{t}\left\|\Delta_{Z} u(s)\right\|_{L^{2}} d s \\
& \leq K\left\|u_{0}\right\|_{L^{2}}+\|V\|_{L^{\infty, \infty}} \int_{0}^{t}\|u(s)\|_{Z} d s,
\end{aligned}
$$

onde $K=K\left(T^{*}\left(u_{0}\right),\|V\|_{L^{\infty, \infty}},\left\|V_{s}\right\|_{L^{\infty, \infty}}, m\right)$. Logo, pela desigualdade de Gronwall

$$
\|u(t)\|_{Z} \leq K\left\|u_{0}\right\|_{L^{2}} e^{\|V\|_{L} \infty, \infty T^{*}\left(u_{0}\right)}<\infty .
$$

Portanto, pela alternativa blow-up, $T^{*}=\infty$. Isto completa a demonstração. 


\section{Capítulo 3}

\section{Propriedades de continuação única}

Neste capítulo seguiremos a técnica usada por Escauriaza, Kenig, Ponce e Vega em [8] para demonstrar algumas propriedades de continuação única para as soluções da equação (2.1). Eles construem duas funções, denotadas aqui por $\varphi$ e $\psi$, as quais são básicas para a obtenção de seus resultado. Nós decidimos colocar estas construções neste capítulo, seção 3.1, e não nas preliminares, porque são importante algumas observações determinantes nas demonstrações dos Teoremas de continuação única 3.10 e 3.14.

Como uma ferramenta fundamental em nosso análise, na seção 3.2, vamos mostrar a existência de um subespaço de funções de $D\left(-\Delta_{Z}\right)$, que tem suporte compacto e infinitamente diferenciáveis para $x \neq 0$ possivelmente. Denotaremos este subespaço por Y. Como uma consequência deste resultado, poderemos aproximar as funções em $C\left([A, B]: D\left(-\Delta_{Z}\right)\right)$ por funções do espaço $C^{1}([A, B]: \mathbf{Y})$. Ainda mais, com a ajuda das funções em $\mathbf{Y}$ e as funções $\varphi$ e $\psi$, na seção 3.3 , conseguimos determinar uma expressão alternativa para $\langle\mathcal{S} f, \mathcal{A} f\rangle+\langle\mathcal{A} f, \mathcal{S} f\rangle$, onde $\mathcal{S}$ é um operador simétrico e $\mathcal{A}$ um operador antisimétrico, a saber,

$$
\begin{aligned}
\langle\mathcal{S} f, \mathcal{A} f\rangle+\langle\mathcal{A} f, \mathcal{S} f\rangle & =2 Z \lambda \varphi^{\prime \prime}(0)|f(0)|^{2} \\
& +\int\left(4 \lambda \varphi^{\prime \prime}\left|\partial_{x} f(x)\right|^{2}-\lambda \varphi^{(i v)}|f(x)|^{2}+4 \lambda^{3}\left(\varphi^{\prime}\right)^{2} \varphi^{\prime \prime}|f(x)|^{2}\right) d x .
\end{aligned}
$$

A identidade dada em (3.1) é fundamental para obter nossos resultados de continuação única, o uso dela é o que nos força a trabalhar por separado em casos, $Z \geq 0$ e $Z<0$.

Notamos que uma estimativa similar a (3.1) foi obtida em [8] através de estimativas associadas ao comutador de $\mathcal{S}$ e $\mathcal{A},[\mathcal{S}, \mathcal{A}]$. A técnica usada em [8] não pode ser de fato aplicada em nosso caso para obter $(3.1)$, pois o domínio específico $D\left(-\Delta_{Z}\right)$ induce restrições a $f \in D\left(-\Delta_{Z}\right)$., como por exemplo, que $f(0, t)=0$ para todo $t \in[0, \infty)$.

\subsection{Existência e propriedades das funções básicas $\varphi$ e $\psi$}

Nesta seção estabelecemos a construção das duas funções $\varphi$ e $\psi$, que irão ser essenciais na deduções da expressão (3.1) e nas demonstrações dos Teoremas 3.10 e 3.14, respectivamente.

Proposição 3.1 Fixemos $\alpha \in[0,1 / 2)$. Então para $p=(4-2 \alpha) / 3 \in(1,4 / 3]$ existe uma função $\varphi=\varphi_{p}$ de classe $C^{4}(\mathbb{R})$, par, estritamente convexa em conjuntos compactos de $\mathbb{R}$, tal que

1. $\varphi(r)=r^{p}+\beta$, para $r=|x| \geq 1$, e $\beta$ uma constante dependendo de $p$

2. $\varphi(0)=0, \varphi(r)>0$, para $r>0$, 
3. existe $M>0$ tal que $\varphi(r) \leq M r^{p}$, para todo $r \in[0, \infty)$.

Demonstração. Para mostrar a existência desta função, suponha que para $r=|x| \leq 1$ a função é o polinômio $\varphi(r)=a_{1} r^{2}+a_{2} r^{4}+a_{3} r^{6}+a_{4} r^{8}$, e para $r=|x| \geq 1$ é a função $\varphi(r)=r^{p}+\beta$ e calculemos coeficientes do polinômio e a constante $\beta$ para que em $x=1$, onde estamos colando, a função seja ao menos de classe $C^{4}(\mathbb{R})$. De fato, devemos então ter

$$
\left\{\begin{array}{rlrl}
a_{1}+a_{2}+a_{3}+a_{4} & =\varphi(1) & =1+\beta, \\
2 a_{1}+4 a_{2}+6 a_{3}+8 a_{4} & =\varphi^{\prime}(1) & =p \\
2 a_{1}+12 a_{2}+30 a_{3}+56 a_{4} & =\varphi^{\prime \prime}(1) & =p(p-1), \\
24 a_{2}+120 a_{3}+336 a_{4} & =\varphi^{\prime \prime \prime}(1) & & =p(p-1)(p-2), \\
24 a_{2}+360 a_{3}+1680 a_{4} & =\varphi^{(i v)}(1) & =p(p-1)(p-2)(p-3) .
\end{array}\right.
$$

Resolvendo este sistemas de equações,

$$
\begin{aligned}
a_{1}=a_{1}(p) & =-\frac{1}{96}(p-8)(p-6)(p-4) p=\frac{1}{96} p\left(-96+76 p-16 p^{2}+p^{3}\right) \\
a_{2}=a_{2}(p) & =\frac{1}{64}(p-8)(p-6)(p-2) p \\
a_{3}=a_{3}(p) & =-\frac{1}{96}(p-8)(p-4)(p-2) p \\
a_{4}=a_{4}(p) & =\frac{1}{384}(p-6)(p-4)(p-2) p \\
\beta=\beta(p) & =-\frac{1}{384}(p-8)(p-6)(p-4)(p-2) \\
& =\frac{1}{384}\left(-384+400 p-140 p^{2}+20 p^{3}-p^{4}\right) .
\end{aligned}
$$

Agora, vamos mostrar que $\varphi$ é uma função convexa no intervalo $[0,1]$, isto é, mostremos que a segunda derivada $\varphi^{\prime \prime}$ é positiva neste intervalo. Em efeito, já que $p \in(1,4 / 3]$, a terceira derivada de $\varphi$,

$$
\begin{aligned}
\varphi^{\prime \prime \prime}(r) & =24 r\left(a_{2}+5 a_{3} r^{2}+14 a_{4} r^{4}\right) \\
& =24 r \frac{p(p-1)}{12 \cdot 16}\left[3(p-6)(p-8)-10(p-4)(p-8) r^{2}+7(p-4)(p-6) r^{4}\right],
\end{aligned}
$$

não muda de sinal no intervalo $(0,1)$, pois o discriminante $\mathcal{D}$ do polinômio quadrático na variável $r^{2}$ em (3.3),

$$
\begin{aligned}
\mathcal{D} & =10^{2}(p-4)^{2}(p-8)^{2}-84(p-6)^{2}(p-8)(p-4), \\
& =4(p-8)(p-4)\left[25(p-4)(p-8)-21(p-6)^{2}\right], \\
& =4(p-8)(p-4)(p-11)(p-1),
\end{aligned}
$$

é negativo. Portanto, no intervalo $(0,1)$ a segunda derivada $\varphi^{\prime \prime}$ ou é estritamente crescente ou é estritamente decrescente.

De outro lado, observe

$$
\varphi^{\prime \prime}(1)=p(p-1)<p<2 a_{1}=\varphi^{\prime \prime}(0) .
$$

Contudo, $\varphi^{\prime \prime} \geq p(p-1)>0$ em $[0, r]$, sendo assim positiva neste intervalo.

Para mostrar o item 3. da proposição, observemos que se $\zeta(r)=M_{0} r^{p}$, com $M_{0}>0$ tal que

$$
M_{0} p(p-1) \geq \sup _{0 \leq r \leq 1}\left|\varphi^{\prime \prime}(r)\right|
$$


então

$$
\zeta^{\prime \prime}(r)=M_{0} p(p-1) r^{p-2} \geq M_{0} p(p-1) \geq \sup _{0 \leq r \leq 1}\left|\varphi^{\prime \prime}(r)\right| \geq \varphi^{\prime \prime}(r),
$$

para todo $r \in[0,1]$. Como

$$
\varphi(0)=\varphi^{\prime}(0)=\zeta(0)=\zeta^{\prime}(0)=0,
$$

obtemos que $\zeta^{\prime}(r) \geq \varphi^{\prime}(r)$ o que imediatamente mostra que $\zeta(r) \geq \varphi(r)$ para todo $r \in[0,1]$. Em razão disto, e do fato que $\beta$ é um número negativo no intervalo aberto $(-1,0)$, escolhemos $M=\max \left\{M_{0}, 1\right\}$ para obter

$$
\varphi(r) \leq M r^{p}, \quad \forall r \geq 0
$$

como se desejava mostrar.

Proposição 3.2 Existe uma função $\psi$ de classe $C^{4}(\mathbb{R})$, par, estritamente convexa em conjuntos compactos de $\mathbb{R}$, tal que

1. $\psi(r)=3 r-\int_{1}^{r} \frac{d s}{1+\log s}+\beta$, para $r=|x| \geq 1$, e $\beta$ uma constante,

2. $\psi(0)=0, \psi(r)>0$, para $r>0$,

3. existe $M>0$ tal que $\psi(r) \leq M r^{p}$, para todo $r \in[0, \infty)$.

Demonstração. A demonstração desta proposição é similar à anterior, propondo para os $r=|x| \leq 1$ o polinômio $\psi(r)=a_{1} r^{2}+a_{2} r^{4}+a_{3} r^{6}+a_{4} r^{8}$, e calculando as constantes $\beta$, $a_{1}, \ldots, a_{4}$, para que o colagem com a função $\psi(r)=3 r-\int_{1}^{r} \frac{d s}{1+\log s}+\beta$, para $r=|x| \geq 1$, em $r=|x|=1$ seja de classe $C^{4}$. Para isto, se resolve um sistema de equações lineares similar ao dado em (3.2), mas tendo em conta que $\psi(1)=3+\beta, \psi^{\prime}(1)=2, \psi^{\prime \prime}(1)=1, \psi^{\prime \prime \prime}(1)=-3$ e $\psi^{(i v)}(1)=14$. Logo, para $r \in[0,1]$, obtemos o polinômio

$$
\psi(r)=\frac{103}{96} r^{2}+\frac{9}{64} r^{4}-\frac{17}{96} r^{6}+\frac{17}{384} r^{8} .
$$

Um calculo direto mostra que a segunda derivada $\psi^{\prime \prime}$ é positiva para $r \geq 1$. Por outro lado, devido a que $\psi^{\prime \prime \prime}(r)=\frac{r}{8}\left(27-170 r^{2}+119 r^{4}\right)$ no intervalo $(0,1)$ tem uma única raíz, a saber, $r_{0}^{2}=\frac{170-\sqrt{16048}}{238}<1 / 4$ e observando que $\psi^{\prime \prime}(0)=\frac{103}{48}, \psi^{\prime \prime}(1)=1$, e $\psi^{\prime \prime}\left(r_{0}\right) \geq 1393 / 768$ podemos concluir que $\psi^{\prime \prime}$ além de ser positiva em $[0,1]$, atinge seu valor mínimo $r=1$.

Finalmente, um argumento similar usado na parte final da proposição 3.1 mostra que

$$
\psi(r) \leq M r, \quad \forall r \geq 0
$$

\subsection{Densidade}

O objetivo desta seção é mostrar que no espaço $D\left(-\Delta_{Z}\right)$, existe um subconjunto denso de funções suaves exceto, possivelmente, em $x=0$ e que tem suporte compacto, considerando a norma $\|\cdot\|_{H^{2}(\mathbb{R} \backslash\{0\})}$. A estratégia é usar a representação das funções $d$ em $D\left(-\Delta_{Z}\right)$, na forma $d=d_{1}+d_{2}$, onde a função $d_{1} \in H^{2}(\mathbb{R})$ e anula-se em zero, e onde $d_{2}$ da o salto característico das funções em $D\left(-\Delta_{Z}\right)$. Logo depois, aproximamos a função $d_{1}$ por funções do conjunto $C_{0}^{\infty}(\mathbb{R} \backslash\{0\})$ e a função $d_{2}$ por uma função de suporte compacto e infinitamente diferenciável exceto, possivelmente, em $x=0$. 
Proposição 3.3 Dada uma função $\vartheta \in W^{m, p}(\mathbb{R})$, com $\vartheta(0)=0$, existe uma sequência de funções $\left(\psi_{n}\right)_{n \geq 1}$ no espaço das funções suaves com suporte compacto, $C_{0}^{\infty}(\mathbb{R})$, satisfazendo $\psi_{n}(0)=0$ para todo $n \in \mathbb{N}$ e convergindo a $\vartheta$ na norma $W^{m, p}(\mathbb{R})$.

Demonstração. Para a demonstração deste fato ver o Apêndice B.1.

Proposição 3.4 Dada uma função $\vartheta \in D\left(-\Delta_{Z}\right)$, existe uma sequência $\left(\vartheta_{n}\right)_{n \geq 1}$ em $D\left(-\Delta_{Z}\right)$ de funções infinitamente diferenciáveis para todo $x \in \mathbb{R}$, exceto possivelmente em $x=0$, $e$ de suporte compacto tal que

$$
\left\|\vartheta-\vartheta_{n}\right\|_{H^{2}(\mathbb{R} \backslash\{0\})} \rightarrow 0
$$

quando $n \rightarrow \infty$.

Demonstração. Seja $\vartheta \in D\left(-\Delta_{Z}\right)$. Na seção 1.1 observamos que para cada uma destas funções, existem uma função $\kappa \in H^{2}(\mathbb{R})$, com $\kappa(0)=0$, uma constante $c \in \mathbb{C}$ e um número real $\theta \in[0,2 \pi)$, tal que

$$
\vartheta=\kappa+c \psi_{i}+c e^{i \theta} \psi_{-i},
$$

onde $\psi_{ \pm i}(x)=\frac{i}{2 \sqrt{ \pm i}} e^{i \sqrt{ \pm i}|x|}$, com $\operatorname{Im} \sqrt{ \pm i}>0$, e a relação entre $Z$ e $\theta$ vem dada pela relação (1.8). Pela Proposição 3.3 , existe uma sequência $\left(\kappa_{n}\right)_{n \geq 1}$ no espaço $C_{0}^{\infty}(\mathbb{R})$ satisfazendo $\kappa_{n}(0)=0 \mathrm{e}$

$$
\left\|\kappa-\kappa_{n}\right\|_{H^{2}} \longrightarrow \infty, \text { quando } n \longrightarrow \infty .
$$

Por outro lado, para cada $n \in \mathbb{Z}^{+}$, consideremos a função $\tau_{n}(x)=\varrho\left(\frac{x}{n}\right)$ onde $\varrho \in C_{0}^{\infty}(\mathbb{R})$ tem imagem contida no intervalo $[0,1]$, é igual a 1 sobre o conjunto $\{x \in \mathbb{R}:|x| \leq 1\}$ e zero sobre $\{x \in \mathbb{R}:|x| \geq 2\}$. Então, a sequência de funções $\left(w_{n}\right)_{n \geq 1}$, onde $w_{n}=\left(c \psi_{i}+c e^{i \theta} \psi_{-i}\right) \tau_{n}$ é contínua, tem suporte compacto em $\mathbb{R}$, é de classe $C^{\infty}$ para todo $x \neq 0$ e a sua derivada satisfaz a mesma condição de salto em $x=0$ como a função $c \psi_{i}+c e^{i \theta} \psi_{-i}$. Já que as sequências $\left(w_{n}\right)_{n \geq 1},\left(\partial_{x} w_{n}\right)_{n \geq 1}$ e $\left(-\Delta_{Z} w_{n}\right)_{n \geq 1}$ convergem as funções $c \psi_{i}+c e^{i \theta} \psi_{-i}, \partial_{x}\left(c \psi_{i}+c e^{i \theta} \psi_{-i}\right)$ e $-\Delta_{Z}\left(c \psi_{i}+c e^{i \theta} \psi_{-i}\right)$ q. t. p., respectivamente, o Teorema da Convergência dominada de Lebesgue mostra que

$$
\left\|c \psi_{i}+c e^{i \theta} \psi_{-i}-w_{n}\right\|_{H^{2}(\mathbb{R} \backslash\{0\})} \longrightarrow 0, \quad \text { quando } n \longrightarrow \infty .
$$

Agora, para cada $n \in \mathbb{Z}^{+}$, definimos $\vartheta_{n} \equiv \kappa_{n}+w_{n}=\kappa_{n}+\left(c \psi_{i}+c e^{i \theta} \psi_{-i}\right) \tau_{n}$. Observemos que cada função $\vartheta_{n}$ tem suporte compacto, é infinitamente diferenciável para todo $x \in \mathbb{R}$ exceto para $x \neq 0$ e $\left(\vartheta_{n}\right)_{n \geq 1} \subset D\left(-\Delta_{Z}\right)$. Além disso,

$$
\left\|\vartheta-\vartheta_{n}\right\|_{H^{2}(\mathbb{R} \backslash\{0\})} \leq\left\|\kappa-\kappa_{n}\right\|_{H^{2}}+\left\|c \psi_{i}+c e^{i \theta} \psi_{-i}-w_{n}\right\|_{H^{2}(\mathbb{R} \backslash\{0\})} \rightarrow 0,
$$

quando $n \rightarrow \infty$, o que mostra o Corolário.

Definição 3.5 Notemos por $\mathbf{Y}$ o subespaço de $D\left(-\Delta_{Z}\right)$, de funções infinitamente diferenciáveis exceto possivelmente para $x \neq 0$ e de suporte compacto.

O seguinte corolário será usado na próxima seção.

Corolário 3.6 Sejam $a, b \in \mathbb{R}$ e $u \in C\left([a, b]: D\left(-\Delta_{Z}\right)\right) \cap C^{1}\left([a, b]: L^{2}(\mathbb{R})\right)$. Então para $n>0$, existe uma sequência de funções $u_{n} \in C_{0}^{\infty}([a, b]: \mathbf{Y})$, tal que

$$
\begin{aligned}
\left\|u(t)-u_{n}(t)\right\|_{H^{2}(\mathbb{R} \backslash\{0\})} & \rightarrow 0, \\
\int_{a}^{b}\left\|u(t)-u_{n}(t)\right\|_{H^{2}(\mathbb{R} \backslash\{0\})} d t & \rightarrow 0, \\
\int_{a}^{b}\left\|\partial_{t} u(t)-\partial_{t} u_{n}(t)\right\|_{L^{2}} d t & \rightarrow 0,
\end{aligned}
$$

quando $n \rightarrow \infty$.

Para a demonstração deste Corolário ver o Apêndice B.2. 


\subsection{Operadores simétricos e antisimétricos auxiliares}

Vamos neste momento usar as funções $\varphi$ e $\psi$ construídas na seção 3.1, com o fim de obter pares de operadores $(\mathcal{S}, \mathcal{A})$, com $\mathcal{S}$ simétrico e $\mathcal{A}$ antisimétrico.

Consideremos a função $f \in C_{0}^{\infty}(\mathbb{R}: \mathbf{Y})$. Então, para cada $t \in[a, b]$ fixo, a função $f(x, t)$ tem suporte compacto e infinitamente diferenciável exceto possivelmente para $x \neq 0$. Também, notemos que para $\lambda>0$ e a função $\varphi$ construída na Proposição 3.1, a função $e^{-\lambda \varphi(x)} f(x, t)$ pertence ao espaço $D\left(-\Delta_{Z}\right)$, pois $\varphi$ anulam-se em $x=0$. Assim $-\Delta_{Z}\left(e^{-\lambda \varphi(x)} f(x, t)\right)=-\partial_{x}^{2}\left(e^{-\lambda \varphi(x)} f(x, t)\right)$ para $x \neq 0$, e temos

$$
\begin{aligned}
e^{\lambda \varphi}\left(\partial_{t}-i \Delta_{Z}\right)\left(e^{-\lambda \varphi} f\right) & =e^{\lambda \varphi}\left(\partial_{t}-i \partial_{x}^{2}\right)\left(e^{-\lambda \varphi} f\right) \\
& =\partial_{t} f-i e^{\lambda \varphi} \partial_{x}\left(-\lambda \varphi^{\prime} f e^{-\lambda \varphi}+e^{-\lambda \varphi} \partial_{x} f\right) \\
& =\partial_{t} f-i\left(-\lambda \varphi^{\prime \prime} f-2 \lambda \varphi^{\prime} \partial_{x} f+\lambda^{2}\left(\varphi^{\prime}\right)^{2} f+\partial_{x}^{2} f\right) \\
& =\partial_{t} f-\mathcal{S}_{1} f-\mathcal{A}_{11} f
\end{aligned}
$$

onde $\mathcal{S}$ é um operador simétrico e $\mathcal{A}_{11}$ é um operador anti-simétrico, sendo os ambos operadores independentes da variável $t$, e definidos como

$$
\left\{\begin{aligned}
\mathcal{S}_{1} & =-i \lambda\left(2 \varphi^{\prime} \frac{d}{d x}+\varphi^{\prime \prime}\right) \\
D\left(\mathcal{S}_{1}\right) & =\left\{g \in H^{1}(\mathbb{R}): \varphi^{\prime} \frac{d g}{d x} \in L^{2}(\mathbb{R})\right\}
\end{aligned}\right.
$$

$\mathrm{e}$

$$
\left\{\begin{aligned}
\mathcal{A}_{11} & =i\left(\Delta_{Z}+\lambda^{2}\left(\varphi^{\prime}\right)^{2}\right) ; \\
D\left(\mathcal{A}_{11}\right) & =\left\{g \in D\left(-\Delta_{Z}\right):\left(\varphi^{\prime}\right)^{2} g \in L^{2}(\mathbb{R})\right\} .
\end{aligned}\right.
$$

Por outro lado, para $V_{2} \in L^{\infty}(\mathbb{R} \times[0,+\infty))$, uma função valor real, procedemos da mesma forma como em (3.4) para obter,

$$
e^{\lambda \varphi}\left(\partial_{t}-i \Delta_{Z}-i V_{2}\right)\left(e^{-\lambda \varphi} f\right)=\partial_{t} f-\mathcal{S}_{1} f-\mathcal{A}_{11} f-i V_{2} f ; \quad x \neq 0
$$

onde $\mathcal{S}_{1}$ e $\mathcal{A}_{11}$ são os operadores definidos em (3.5) e (3.6). Defina $\mathcal{A}_{12}=\mathcal{A}_{11}+i V_{2}$, que pelas condições de limitação de $V_{2}$ tem o mesmo domínio que o operador $\mathcal{A}_{11}$, isto é, $D\left(\mathcal{A}_{12}\right)=D\left(\mathcal{A}_{11}\right)$, e além disso, é um operador antisimétrico.

Se trocamos a função $\varphi$ pela função $\psi$ construída na Proposição 3.2, e como esta função tem derivadas $\psi^{\prime}$ e $\psi^{\prime \prime}$ limitadas, obtemos os seguintes operadores independentes do tempo

$$
\left\{\begin{aligned}
\mathcal{S}_{2} & =-i \lambda\left(2 \psi^{\prime} \frac{d}{d x}+\psi^{\prime \prime}\right) \\
D\left(\mathcal{S}_{2}\right) & =H^{1}(\mathbb{R})
\end{aligned}\right.
$$

o qual é simétrico, e

$$
\left\{\begin{aligned}
\mathcal{A}_{21} & =i\left(\Delta_{Z}+\lambda^{2}\left(\psi^{\prime}\right)^{2}\right) \\
D\left(\mathcal{A}_{21}\right) & =D\left(-\Delta_{Z}\right)
\end{aligned}\right.
$$

o qual é antisimétrico, ainda mais, e o operador

$$
\mathcal{A}_{22}=\mathcal{A}_{21}+i V_{2}
$$

também é antisimétrico e com domínio $D\left(\mathcal{A}_{22}\right)=D\left(\mathcal{A}_{21}\right)$.

Com respeito a estes operadores vamos enunciar as seguintes duas proposições que serão básicas para a demonstração dos Lemas 3.11, 3.15, 3.12 e 3.16 abaixo. 
Proposição 3.7 Seja $T_{0}, T_{1} \in \mathbb{R}, T_{0}<T_{1}$, e suponha que $f$ e uma função no espaço $C\left([0,+\infty): D\left(\mathcal{S}_{k}\right) \cap D\left(\mathcal{A}_{k j}\right)\right) \cap C^{1}\left((0,+\infty): L^{2}(\mathbb{R})\right), k, j=1,2$, então temos

$$
\begin{aligned}
& \int_{T_{0}}^{T_{1}}\left\langle\mathcal{S}_{k} f, \mathcal{A}_{k j} f\right\rangle+\left\langle\mathcal{A}_{k j} f, \mathcal{S}_{k} f\right\rangle d t+\int_{T_{0}}^{T_{1}} \int\left|\mathcal{S}_{k} f\right|^{2} d x d t \\
& \quad \leq \int_{T_{0}}^{T_{1}} \int\left|\partial_{t} f-\left(\mathcal{S}_{k}+\mathcal{A}_{k j}\right) f\right|^{2} d x d t+\left|\int \mathcal{S}_{k} f\left(T_{1}\right) \overline{f\left(T_{1}\right)} d x\right|+\left|\int \mathcal{S}_{k} f\left(T_{0}\right) \overline{f\left(T_{0}\right)} d x\right|
\end{aligned}
$$

Demonstração. Observe

$$
\begin{aligned}
\partial_{t}\left\langle\mathcal{S}_{k} f, f\right\rangle & =2 \operatorname{Re}\left\langle\partial_{t} f-\left(\mathcal{S}_{k}+\mathcal{A}_{k j}\right) f, \mathcal{S}_{k} f\right\rangle+2\left\langle\mathcal{S}_{k} f, \mathcal{S}_{k} f\right\rangle \\
& +\left\langle\mathcal{S}_{k} f, \mathcal{A}_{k j} f\right\rangle+\left\langle\mathcal{A}_{k j} f, \mathcal{S}_{k} f\right\rangle
\end{aligned}
$$

Logo integrando no intervalo de tempo $\left[T_{0}, T_{1}\right]$ segue-se

$$
\begin{aligned}
\int_{T_{0}}^{T_{1}}\left\langle\mathcal{S}_{k} f, \mathcal{A}_{k j} f\right\rangle & +\left\langle\mathcal{A}_{k j} f, \mathcal{S}_{k} f\right\rangle d t+2 \int_{T_{0}}^{T_{1}}\left\langle\mathcal{S}_{k} f, \mathcal{S}_{k} f\right\rangle d t \\
& =-2 \operatorname{Re} \int_{T_{0}}^{T_{1}}\left\langle\partial_{t} f-\left(\mathcal{S}_{k}+\mathcal{A}_{k j}\right) f, \mathcal{S}_{k} f\right\rangle d t+\left.\left\langle\mathcal{S}_{k} f, f\right\rangle\right|_{T_{0}} ^{T_{1}} \\
& \leq 2 \int_{T_{0}}^{T_{1}}\left|\left\langle\partial_{t} f-\left(\mathcal{S}_{k}+\mathcal{A}_{k j}\right) f, \mathcal{S}_{k} f\right\rangle\right| d t+\left|\left\langle\mathcal{S}_{k} f, f\right\rangle\right|_{T_{0}}^{T_{1}} \mid
\end{aligned}
$$

Assim,a conclusão da Proposição é obtida só observando que para $a, b$ números reais, $a b \leq$ $\left(a^{2}+b^{2}\right) / 2$.

No enunciado da próxima proposição vamos precisar da função $\eta:\left[T-\frac{1}{2}, T+\frac{1}{2}\right] \rightarrow \mathbb{R}$ definida como

$$
\eta(t)=\left[t-\left(T-\frac{1}{2}\right)\right]\left[\left(T+\frac{1}{2}\right)-t\right]
$$

onde $T \geq 1 / 2$ é fixo. É fácil ver que $\eta$ satisfaz:

1. $\eta\left(T-\frac{1}{2}\right)=\eta\left(T+\frac{1}{2}\right)=0, \eta \prime\left(T-\frac{1}{2}\right)=1$ e $\eta \prime\left(T+\frac{1}{2}\right)=-1$

2. para qualquer $t \in\left[T-\frac{1}{2}, T+\frac{1}{2}\right]$,

$$
0 \leq \eta(t) \leq \frac{1}{4}, \quad\left|\eta^{\prime}(t)\right| \leq 1, \quad \eta^{\prime \prime}=-2
$$

Proposição 3.8 Para qualquer $T>\frac{1}{2}$ e $f \in C\left([0,+\infty): D\left(\mathcal{A}_{k j}^{\prime}\right)\right) \cap C^{1}\left((0,+\infty): L^{2}(\mathbb{R})\right)$, $k, j=1,2$ então temos

$$
\begin{aligned}
& 2 \int_{T-\frac{1}{2}}^{T+\frac{1}{2}} \eta(t)\left(\left\|\mathcal{S}_{k} f\right\|_{L^{2}(\mathbb{R})}^{2}+\left\langle\mathcal{S}_{k} f, \mathcal{A}_{k j} f\right\rangle+\left\langle\mathcal{A}_{k j} f, \mathcal{S}_{k} f\right\rangle\right) d t+\int_{T-\frac{1}{2}}^{T+\frac{1}{2}} \int|f|^{2} d x d t \\
& \quad \leq 3 \int_{T-\frac{1}{2}}^{T+\frac{1}{2}} \int\left|\partial_{t} f-\left(\mathcal{S}_{k}+\mathcal{A}_{k j}\right) f\right|^{2} d x d t+\left\|f\left(T-\frac{1}{2}\right)\right\|_{L^{2}(\mathbb{R})}^{2}+\left\|f\left(T+\frac{1}{2}\right)\right\|_{L^{2}(\mathbb{R})}^{2}
\end{aligned}
$$

Demonstração. Como $\mathcal{A}_{k j}$ é um operador anti-simétrico, se tem $\left\langle\mathcal{A}_{k j} f, f\right\rangle+\left\langle f, \mathcal{A}_{k j} f\right\rangle=0$, portanto

$$
\partial_{t}\langle f, f\rangle=2 \operatorname{Re}\left\langle\partial_{t} f-\left(\mathcal{S}_{k}+\mathcal{A}_{k j}\right) f, f\right\rangle+2\left\langle\mathcal{S}_{k} f, f\right\rangle .
$$


Multiplicando por $\eta \prime(t)$ e integrando sobre o intervalo de tempo $\left[T-\frac{1}{2}, T+\frac{1}{2}\right]$ a anterior igualdade, obtemos

$$
\begin{aligned}
\int_{T^{-\frac{1}{2}}}^{T+\frac{1}{2}} \eta \prime(t) \partial_{t}\langle f, f\rangle d t & =2 \operatorname{Re} \int_{T^{-\frac{1}{2}}}^{T+\frac{1}{2}} \eta \prime(t)\left\langle\partial_{t} f-\left(\mathcal{S}_{k}+\mathcal{A}_{k j}\right) f, f\right\rangle d t \\
& +2 \int_{T^{-\frac{1}{2}}}^{T+\frac{1}{2}} \eta \prime(t)\left\langle\mathcal{S}_{k} f, f\right\rangle d t
\end{aligned}
$$

Agora, integrando por partes tanto o termo do lado esquerdo como o último do lado direito da igualdades (3.14) e usando as observações sobre $\eta$ e a sua derivada $\eta$ ' conseguimos que

$$
\begin{aligned}
& -\left\|f\left(T-\frac{1}{2}\right)\right\|_{L^{2}(\mathbb{R})}^{2}-\left\|f\left(T+\frac{1}{2}\right)\right\|_{L^{2}(\mathbb{R})}^{2}+2 \int_{T^{-\frac{1}{2}}}^{T+\frac{1}{2}}\langle f, f\rangle d t \\
& =2 \operatorname{Re} \int_{T^{-\frac{1}{2}}}^{T+\frac{1}{2}} \eta \prime(t)\left\langle\partial_{t} f-\left(\mathcal{S}_{k}+\mathcal{A}_{k j}\right) f, f\right\rangle d t-2 \int_{T^{-\frac{1}{2}}}^{T+\frac{1}{2}} \eta(t) \partial_{t}\left\langle\mathcal{S}_{k} f, f\right\rangle d t .
\end{aligned}
$$

Agora, substituído (3.12) na última integral da igualdade (3.15) e reorganizando, obtemos

$$
\begin{aligned}
& 2 \int_{T^{-\frac{1}{2}}}^{T+\frac{1}{2}} \eta(t)\left(\left\langle\mathcal{S}_{k} f, \mathcal{A}_{k j} f\right\rangle+\left\langle\mathcal{A}_{k j} f, \mathcal{S}_{k} f\right\rangle\right) d t+4 \int_{T^{-\frac{1}{2}}}^{T+\frac{1}{2}} \eta(t)\left\|\mathcal{S}_{k} f(t)\right\|_{L^{2}(\mathbb{R})}^{2} d t+2 \int_{T^{-\frac{1}{2}}}^{T+\frac{1}{2}}\|f\|_{L^{2}(\mathbb{R})}^{2} d t \\
& \quad=2 \operatorname{Re} \int_{T^{-\frac{1}{2}}}^{T+\frac{1}{2}} \eta \prime(t)\left\langle\partial_{t} f-\left(\mathcal{S}_{k}+\mathcal{A}_{k j}\right) f, f\right\rangle d t-4 R e \int_{T^{-\frac{1}{2}}}^{T+\frac{1}{2}} \eta(t)\left\langle\partial_{t} f-\left(\mathcal{S}_{k}+\mathcal{A}_{k j}\right) f, \mathcal{S}_{k} f\right\rangle d t \\
& \quad+\left\|f\left(T-\frac{1}{2}\right)\right\|_{L^{2}(\mathbb{R})}^{2}+\left\|f\left(T+\frac{1}{2}\right)\right\|_{L^{2}(\mathbb{R})}^{2},
\end{aligned}
$$

daí segue-se a desigualdade (3.13).

Observação 3.9 Claramente, para $\xi \in C_{0}^{4}(\mathbb{R}: \mathbf{Y})$ temos $\xi(t) \in D\left(\mathcal{A}_{k j}\right) \cap D\left(\mathcal{S}_{k}\right), k, j=1,2$. Logo, temos para os operadores $\mathcal{S}_{1}$ e $\mathcal{A}_{11}$, a seguinte identidade:

$$
\begin{aligned}
\left\langle\mathcal{S}_{1} \xi(t), \mathcal{A}_{11} \xi(t)\right\rangle & +\left\langle\mathcal{A}_{11} \xi(t), \mathcal{S}_{1} \xi(t)\right\rangle=2 Z \lambda \varphi^{\prime \prime}(0)|\xi(0, t)|^{2} \\
& +\int\left(4 \lambda \varphi^{\prime \prime}\left|\partial_{x} \xi(t)\right|^{2}-\lambda \varphi^{(i v)}|\xi(t)|^{2}+4 \lambda^{3}\left(\varphi^{\prime}\right)^{2} \varphi^{\prime \prime}|\xi(t)|^{2}\right) d x .
\end{aligned}
$$

(ver apêndice B.3 para a demonstração desta identidade). Também, para os operadores $\mathcal{S}_{1}$ e $\mathcal{A}_{12}$ temos

$$
\begin{aligned}
\left\langle\mathcal{S}_{1} \xi(t), \mathcal{A}_{12} \xi(t)\right\rangle & +\left\langle\mathcal{A}_{12} \xi(t), \mathcal{S}_{1} \xi(t)\right\rangle=\left\langle\mathcal{S}_{k} \xi(t), \mathcal{A}_{1} \xi(t)\right\rangle+\left\langle\mathcal{A}_{1} \xi(t), \mathcal{S}_{k} \xi(t)\right\rangle \\
& +\left\langle\mathcal{S}_{k} \xi(t), i V_{2} \xi(t)\right\rangle+\left\langle i V_{2} \xi(t), \mathcal{S}_{k} \xi(t)\right\rangle \\
& =2 Z \lambda \varphi^{\prime \prime}(0)|\xi(0, t)|^{2}+2 \lambda \int \varphi^{\prime} \partial_{x} V_{2}|\xi(t)|^{2} d x \\
& +\int\left(4 \lambda \varphi^{\prime \prime}\left|\partial_{x} \xi(t)\right|^{2}-\lambda \varphi^{(i v)}|\xi(t)|^{2}+4 \lambda^{3}\left(\varphi^{\prime}\right)^{2} \varphi^{\prime \prime}|\xi(t)|^{2}\right) d x
\end{aligned}
$$

Agora, como $\varphi^{\prime} \partial_{x} V_{2}=(d \varphi / d r) \partial_{r} V_{2}$, onde as derivadas à direita desta igualdade são derivadas radiais $(r=|x|)$,

$$
\begin{aligned}
\left\langle\mathcal{S}_{1} \xi(t), \mathcal{A}_{12} \xi(t)\right\rangle & +\left\langle\mathcal{A}_{12} \xi(t), \mathcal{S}_{1} \xi(t)\right\rangle=2 Z \lambda \varphi^{\prime \prime}(0)|\xi(0, t)|^{2}+2 \lambda \int \frac{d \varphi}{d r} \partial_{r} V_{2}|\xi(t)|^{2} d x \\
& +\int\left(4 \lambda \varphi^{\prime \prime}\left|\partial_{x} \xi(t)\right|^{2}-\lambda \varphi^{(i v)}|\xi(t)|^{2}+4 \lambda^{3}\left(\varphi^{\prime}\right)^{2} \varphi^{\prime \prime}|\xi(t)|^{2}\right) d x
\end{aligned}
$$


Analogamente, utilizando os operadores $\mathcal{S}_{2}, \mathcal{A}_{21}$ e $A_{22}$, obtemos as identidades

$$
\begin{aligned}
\left\langle\mathcal{S}_{2} \xi(t), \mathcal{A}_{21} \xi(t)\right\rangle & +\left\langle\mathcal{A}_{21} \xi(t), \mathcal{S}_{2} \xi(t)\right\rangle=2 Z \lambda \psi^{\prime \prime}(0)|\xi(0, t)|^{2} \\
& +\int\left(4 \lambda \psi^{\prime \prime}\left|\partial_{x} \xi(t)\right|^{2}-\lambda \psi^{(i v)}|\xi(t)|^{2}+4 \lambda^{3}\left(\psi^{\prime}\right)^{2} \psi^{\prime \prime}|\xi(t)|^{2}\right) d x
\end{aligned}
$$

$e$

$$
\begin{aligned}
\left\langle\mathcal{S}_{2} \xi(t), \mathcal{A}_{22} \xi(t)\right\rangle & +\left\langle\mathcal{A}_{22} \xi(t), \mathcal{S}_{2} \xi(t)\right\rangle=2 Z \lambda \psi^{\prime \prime}(0)|\xi(0, t)|^{2}+2 \lambda \int \frac{d \psi}{d r} \partial_{r} V_{2}|\xi(t)|^{2} d x \\
& +\int\left(4 \lambda \psi^{\prime \prime}\left|\partial_{x} \xi(t)\right|^{2}-\lambda \psi^{(i v)}|\xi(t)|^{2}+4 \lambda^{3}\left(\psi^{\prime}\right)^{2} \psi^{\prime \prime}|\xi(t)|^{2}\right) d x
\end{aligned}
$$

\subsection{Resultados principais}

Nesta seção estabelecemos o objetivo principal desta tese, a saber, as propriedades de continuação única para soluções da equação

$$
\partial_{t} u=i\left(\Delta_{Z}+V(x, t)\right) u
$$

A continuação nosso primeiro resultado.

Teorema 3.10 Seja $u \in C\left([0, \infty): D\left(-\Delta_{Z}\right)\right) \cap C^{1}\left((0, \infty): L^{2}(\mathbb{R})\right)$ uma solução da equação (3.21) com potencial $V \in L^{\infty}(\mathbb{R} \times[0, \infty))$ satisfazendo

$$
V(x, t)=V_{1}(x, t)+V_{2}(x, t)
$$

com $V_{j}, j=1,2$ de valor real,

$$
\left|V_{1}(x, t)\right| \leq \frac{c_{1}}{\langle x\rangle^{\alpha}}, \quad 0 \leq \alpha<1 / 2,
$$

e $V_{2}$ com suporte em $\{(x, t):|x| \geq 1\}$ tal que

$$
-\left(\partial_{r} V_{2}(x, t)\right)^{-} \leq \frac{c_{2}}{|x|^{\frac{1+4 \alpha}{3}}}, \quad a^{-}=\min \{a ; 0\} .
$$

Então, existe uma constante $\lambda_{s}=\lambda_{s}\left(\|V\|_{L^{\infty}(\mathbb{R} \times[0, \infty))} ; c_{1} ; c_{2} ; \alpha\right)>0$ tal que,

i. se para $Z \geq 0$, temos

$$
\sup _{t \geq 0} \int_{\mathbb{R}} e^{2 M \lambda_{s}|x|^{p}}|u(x, t)|^{2} d x \leq C_{\lambda_{s}}, \quad p=\frac{4-2 \alpha}{3}
$$

ou

ii. se para $Z<0$, temos

$$
\int_{0}^{\infty}|u(0, t)|^{2} d t+\sup _{t \geq 0} \int_{\mathbb{R}} e^{2 M \lambda_{s}|x|^{p}}|u(x, t)|^{2} d x \leq C_{\lambda_{s}}, \quad p=\frac{4-2 \alpha}{3},
$$

onde $M>0$ é a constante dada no item 3. da Proposição 3.1, então

$$
u \equiv 0
$$


Os seguintes dois lemas são peças fundamentais na demonstração deste teorema.

Lema 3.11 Sejam u e $V$ satisfazendo as condições do Teorema 3.10 e seja $\lambda>0$ tal que i. se para $Z \geq 0$, temos

$$
\sup _{t \geq 0} \int_{\mathbb{R}} e^{2 M \lambda|x|^{p}}|u(x, t)|^{2} d x \leq C_{\lambda}, \quad p=\frac{4-2 \alpha}{3},
$$

ou

ii. se para $Z<0$, temos

$$
\int_{0}^{\infty}|u(0, t)|^{2} d t+\sup _{t \geq 0} \int_{\mathbb{R}} e^{2 M \lambda|x|^{p}}|u(x, t)|^{2} d x \leq C_{\lambda}, \quad p=\frac{4-2 \alpha}{3},
$$

onde $0 \leq \alpha<1 / 2, M>0$ é a constante dada no item 3. da Proposição 3.1. Então, existe uma sequência de números reais $\left\{T_{j}: j \in \mathbb{Z}^{+}\right\}$com $T_{j} \uparrow \infty$ quando $j \uparrow \infty$ tal que

$$
\sup _{j \in \mathbb{Z}^{+}} \int_{\mathbb{R}}\left|\mathcal{S}_{1} f\left(x, T_{j}\right)\right|^{2} d x \leq \bar{C}_{\lambda}
$$

onde $f(x, t)=e^{\lambda \varphi(x)} u(x, t)$, e $\mathcal{S}_{1}$ é o operador dado em (3.5). Aqui, quando $Z \geq 0, \bar{C}_{\lambda}=$ $\bar{C}_{\lambda}\left(C_{\lambda}, \lambda,\|V\|_{L^{\infty}}, p\right)$ denota uma constante que depende desses quatro parâmetros. Além disso, se $Z<0$, esta constante, $\bar{C}_{\lambda}$, depende também de $|Z|$.

Demonstração. Antes de começar a demonstração observemos que combinando as hipótese $V \in L^{\infty}(\mathbb{R} \times[0, \infty))$ e a dada em $(3.27)$ obtemos

$$
\sup _{t \geq 0} \int_{\mathbb{R}} e^{2 M \lambda|x|^{p}}|V(x, t) u(x, t)|^{2} d x \leq C_{\lambda}\|V\|_{L^{\infty, \infty}} .
$$

Logo, como $u \in C\left([0, \infty): D\left(-\Delta_{Z}\right)\right) \cap C^{1}\left((0, \infty): L^{2}(\mathbb{R})\right)$ é solução de $(3.21)$, temos

$$
\sup _{t \geq 0} \int_{\mathbb{R}} e^{2 M \lambda|x|^{p}}\left|\partial_{t} u(x, t)-i \Delta_{Z} u(x, t)\right|^{2} d x \leq C_{\lambda}\|V\|_{L^{\infty, \infty}} .
$$

- Caso $Z \geq 0$ e $V_{2} \equiv 0$. Observemos, então que $V=V_{1}$. Já que $u \in C\left([0, \infty): D\left(-\Delta_{Z}\right)\right) \cap$ $C^{1}\left((0, \infty): L^{2}(\mathbb{R})\right)$, para cada $T>1 / 2$ fixo, o Corolário 3.6 implica que existe uma sequência $\left(u_{n}\right)_{n \geq 1}$ contida em $C_{0}^{\infty}\left(\left[T-\frac{1}{2}, T+\frac{1}{2}\right]: \mathbf{Y}\right)$ tal que

$$
\begin{gathered}
\int_{T-\frac{1}{2}}^{T+\frac{1}{2}}\left\{\left\|\partial_{t} u_{n}(t)-\partial_{t} u(t)\right\|_{L^{2}}+\left\|u_{n}(t)-u(t)\right\|_{H^{2}(\mathbb{R} \backslash\{0\})}\right\} d t \longrightarrow 0, \\
\left\|u_{n}(t)-u(t)\right\|_{H^{2}(\mathbb{R} \backslash\{0\})} \rightarrow \infty, \quad \forall t \in\left[T-\frac{1}{2}, T+\frac{1}{2}\right],
\end{gathered}
$$

quando $n \longrightarrow \infty$. Se fixamos na Proposição 3.8, os operadores $\mathcal{S}_{1} \mathcal{A}_{11}$ e considerando a função $f_{n}(x, t)=e^{\lambda \varphi(x)} u_{n}(x, t)$, obtemos, com ajuda da identidade (3.17) trocando $\xi$ por $f_{n}$, a seguinte desigualdade,

$$
\begin{aligned}
& 2 \int_{T-\frac{1}{2}}^{T+\frac{1}{2}} \int_{\mathbb{R}} \eta(t)\left[\left|\mathcal{S}_{1} f_{n}\right|^{2}+4 \lambda^{3}\left(\varphi^{\prime}\right)^{2} \varphi^{\prime \prime}\left|f_{n}\right|^{2}-\lambda \varphi^{(i v)}\left|f_{n}\right|^{2}+4 \lambda \varphi^{\prime \prime}\left|\partial_{x} f_{n}\right|^{2}\right] d x d t \\
& \quad+4 Z \lambda \varphi^{\prime \prime}(0) \int_{T-\frac{1}{2}}^{T+\frac{1}{2}} \eta(t)\left|f_{n}(0, t)\right|^{2} d t+\int_{T-\frac{1}{2}}^{T+\frac{1}{2}} \int_{\mathbb{R}}\left|f_{n}(x, t)\right|^{2} d x d t \\
& \leq 3 \int_{T-\frac{1}{2}}^{T+\frac{1}{2}} \int_{\mathbb{R}}\left|\partial_{t} f_{n}-\left(\mathcal{S}_{k}+\mathcal{A}_{11}\right) f_{n}\right|^{2} d x d t+\int_{\mathbb{R}}\left|f_{n}\left(x, T-\frac{1}{2}\right)\right|^{2} d x+\int_{\mathbb{R}}\left|f_{n}\left(x, T+\frac{1}{2}\right)\right|^{2} d x
\end{aligned}
$$


Da construção de $\varphi$ sabemos que $\varphi^{\prime \prime}$ é positiva e limitada, logo combinando este fato com a identidade (3.4) em relação a função $f_{n}$, e passando o termo que envolve $\varphi^{(i v)}$ à direita da desigualdade, obtemos a seguinte desigualdade,

$$
\begin{aligned}
& 2 \int_{T-\frac{1}{2}}^{T+\frac{1}{2}} \int_{\mathbb{R}} \eta(t)\left|\mathcal{S}_{1} f_{n}\right|^{2} d x d t \leq 3 \int_{T-\frac{1}{2}}^{T+\frac{1}{2}} \int_{\mathbb{R}}\left|\partial_{t} f_{n}-i e^{\lambda \varphi} \Delta_{Z} u_{n}\right|^{2} d x d t \\
&+2 \lambda\left\|\varphi^{(i v)}\right\|_{\infty} \int_{T-\frac{1}{2}}^{T+\frac{1}{2}} \int_{\mathbb{R}}\left|f_{n}(x, t)\right|^{2} d x d t+\int_{\mathbb{R}}\left|f_{n}\left(x, T-\frac{1}{2}\right)\right|^{2} d x \\
&+\int_{\mathbb{R}}\left|f_{n}\left(x, T+\frac{1}{2}\right)\right|^{2} d x-4 Z \lambda \varphi^{\prime \prime}(0) \int_{T-\frac{1}{2}}^{T+\frac{1}{2}} \eta(t)\left|f_{n}(0, t)\right|^{2} d t .
\end{aligned}
$$

Apagando o termo o qual contem a constante $Z$, e tomando o limite quando $n \longrightarrow \infty$, conseguimos

$$
\begin{aligned}
& 2 \int_{T-\frac{1}{2}}^{T+\frac{1}{2}} \int_{\mathbb{R}} \eta(t)\left|\mathcal{S}_{1} f\right|^{2} d x d t \leq 3 \int_{T-\frac{1}{2}}^{T+\frac{1}{2}} \int_{\mathbb{R}}\left|\partial_{t} f-i e^{\lambda \varphi} \Delta_{Z} u\right|^{2} d x d t \\
& \quad+2 \lambda\left\|\varphi^{(i v)}\right\|_{\infty} \int_{T-\frac{1}{2}}^{T+\frac{1}{2}} \int_{\mathbb{R}}|f|^{2} d x d t+\int_{\mathbb{R}}\left|f\left(x, T-\frac{1}{2}\right)\right|^{2} d x+\int_{\mathbb{R}}\left|f\left(x, T+\frac{1}{2}\right)\right|^{2} d x
\end{aligned}
$$

onde $f(x, t)=e^{\lambda \varphi} u(x, t)$. Já que $u(x, t)$ é solução da equação (3.21), segue-se

$$
\begin{aligned}
& 2 \int_{T-\frac{1}{2}}^{T+\frac{1}{2}} \int_{\mathbb{R}} \eta(t)\left|\mathcal{S}_{1} f\right|^{2} d x d t \leq 3 \int_{T-\frac{1}{2}}^{T+\frac{1}{2}} \int_{\mathbb{R}} e^{2 \lambda \varphi(x)}\left|V_{1}(x, t) u(x, t)\right|^{2} d x d t \\
& \quad+2 \lambda\left\|\varphi^{i v}\right\|_{\infty} \int_{T-\frac{1}{2}}^{T+\frac{1}{2}} \int_{\mathbb{R}}|f(x, t)|^{2} d x d t+\int_{\mathbb{R}}\left|f\left(x, T-\frac{1}{2}\right)\right|^{2} d x+\int_{\mathbb{R}}\left|f\left(x, T+\frac{1}{2}\right)\right|^{2} d x \\
& \leq 3\left\|V_{1}\right\|_{L_{x}^{\infty} L_{t}^{\infty}} \sup _{t>0} \int_{\mathbb{R}} e^{2 \lambda \varphi(x)}|u(x, t)|^{2} d x+2 \lambda\left\|\varphi^{(i v)}\right\|_{\infty} \sup _{t>0} \int_{\mathbb{R}}|f(x, t)|^{2} d x \\
& \quad+2 \sup _{t>0} \int_{\mathbb{R}}|f(x, t)|^{2} d x \\
& \leq\left(2+3 c_{1}^{2}+2 \lambda\left\|\varphi^{(i v)}\right\|_{\infty}\right) \sup _{t>0} \int_{\mathbb{R}} e^{2 \lambda \varphi(x)}|u(x, t)|^{2} d x .
\end{aligned}
$$

Usando a hipótese (3.27) conseguimos a seguinte desigualdade

$$
\int_{T-\frac{1}{2}}^{T+\frac{1}{2}} \int_{\mathbb{R}} \eta(t)\left|\mathcal{S}_{1} f\right|^{2} d x d t \leq \bar{C}_{\lambda}\left(C_{\lambda}, c_{1}, p, \lambda\right) .
$$

Agora, observemos que para todo $t \in\left[T-\frac{1}{4}, T+\frac{1}{4}\right]$, temos

$$
\frac{1}{4} \leq t-\left(T-\frac{1}{2}\right) \leq \frac{3}{4}, \quad \frac{1}{4} \leq\left(T+\frac{1}{2}\right)-t \leq \frac{3}{4}
$$

$\log 0$

$$
\frac{1}{16} \leq \eta(t) \leq \frac{9}{16},
$$


e este fato nós permite concluir que

$$
\begin{aligned}
\bar{C}_{\lambda} & \geq \int_{T-\frac{1}{2}}^{T+\frac{1}{2}} \int_{\mathbb{R}} \eta(t)\left|\mathcal{S}_{1} f\right|^{2} d x d t \geq \int_{T-\frac{1}{4}}^{T+\frac{1}{4}} \int_{\mathbb{R}} \eta(t)\left|\mathcal{S}_{1} f\right|^{2} d x d t \\
& \geq \frac{1}{16} \int_{T-\frac{1}{4}}^{T+\frac{1}{4}} \int_{\mathbb{R}}\left|\mathcal{S}_{1} f\right|^{2} d x d t
\end{aligned}
$$

Agora, usando o Teorema do valor intermédio para integrais, temos que para algum $T^{*}$ em $\left[T-\frac{1}{4}, T+\frac{1}{4}\right]$ que

$$
\begin{aligned}
\int_{T-\frac{1}{4}}^{T+\frac{1}{4}} \int_{\mathbb{R}}\left|\mathcal{S}_{1} f\right|^{2} d x d t & =\left(T+\frac{1}{4}-\left(T-\frac{1}{4}\right)\right) \int_{\mathbb{R}}\left|\mathcal{S}_{1} f\left(x, T^{*}\right)\right|^{2} d x \\
& =\frac{1}{2} \int_{\mathbb{R}}\left|\mathcal{S}_{1} f\left(x, T^{*}\right)\right|^{2} d x .
\end{aligned}
$$

Portanto, da combinação das desigualdades (3.35) e (3.36) com a igualdade (3.37) encontramos uma sequência $\left\{T_{j}: j \in \mathbb{Z}^{+}\right\}$com $T_{j} \uparrow \infty$, quando $j \uparrow \infty$ com a propriedade que

$$
\sup _{j \in \mathbb{Z}^{+}} \int_{\mathbb{R}}\left|\mathcal{S}_{1} f\left(x, T_{j}\right)\right|^{2} d x \leq \bar{C}_{\lambda}
$$

- Caso $Z \geq 0$ e $V_{2} \not \equiv 0$. Vamos seguir a mesma linha de argumentação usada no caso $V_{2} \equiv 0$. Isto é, aplicaremos à funções $f_{n}(x, t)=e^{\lambda \varphi} u_{n}(x, t)$ a Proposição 3.8; mas fixando os operadores $\mathcal{S}_{1}$ e $\mathcal{A}_{12}$, e logo usando da identidade dada em (3.18) depois de substituir $\xi$ por $f_{n}$. Assim,

$$
\begin{aligned}
& 2 \int_{T-\frac{1}{2}}^{T+\frac{1}{2}} \int_{\mathbb{R}} \eta(t)\left[\left|\mathcal{S}_{1} f_{n}\right|^{2}+4 \lambda^{3}\left(\varphi^{\prime}\right)^{2} \varphi^{\prime \prime}\left|f_{n}\right|^{2}-\lambda \varphi^{(i v)}\left|f_{n}\right|^{2}+4 \lambda \varphi^{\prime \prime}\left|\partial_{x} f_{n}\right|^{2}\right] d x d t \\
& +4 Z \lambda \varphi^{\prime \prime}(0) \int_{T-\frac{1}{2}}^{T+\frac{1}{2}} \eta(t)\left|f_{n}(0, t)\right|^{2} d t+\int_{T-\frac{1}{2}}^{T+\frac{1}{2}} \int_{\mathbb{R}}\left|f_{n}\right|^{2} d x+2 \lambda \int_{T-\frac{1}{2}}^{T+\frac{1}{2}} \int_{\mathbb{R}} \frac{d \varphi}{d r} \partial_{r} V_{2}\left|f_{n}\right|^{2} d x d t \\
& \leq 3 \int_{T-\frac{1}{2}}^{T+\frac{1}{2}} \int_{\mathbb{R}}\left|\partial_{t} f_{n}-\left(\mathcal{S}_{1}+\mathcal{A}_{12}\right) f_{n}\right|^{2} d x d t+\int_{\mathbb{R}}\left|f_{n}\left(x, T-\frac{1}{2}\right)\right|^{2} d x \\
& +\int_{\mathbb{R}}\left|f_{n}\left(x, T+\frac{1}{2}\right)\right|^{2} d x .
\end{aligned}
$$

Notemos que a diferença entre esta desigualdade e a dada em (3.31) é que aparece o termo

$$
2 \lambda \int_{\mathbb{R}} \frac{d \varphi}{d r} \partial_{r} V_{2}\left|f_{n}\right|^{2} d x
$$

onde $\frac{d \varphi}{d r}$ e $\partial_{r} V_{2}$ são as derivadas radiais de $\varphi$ e $V_{2}$ respectivamente. É fácil verificar que esta integral é limitada inferiormente por

$$
2 \lambda \int_{\mathbb{R}} \frac{d \varphi}{d r}\left(\partial_{r} V_{2}\right)^{-}\left|f_{n}\right|^{2} d x
$$

onde $\left(\partial_{r} V_{2}\right)^{-}=\min \left\{\partial_{r} V_{2}, 0\right\}$. Por outro lado, lembremos que o suporte de $V_{2}(x, t)$ é o conjunto $\{(x, t):|x| \geq 1\}$ e que para $|x| \geq 1$ a derivada $\frac{d \varphi}{d r}=p r^{p-1}$, então da hipótese (3.24) temos

$$
-\frac{d \varphi}{d r}\left(\partial_{r} V_{2}\right)^{-} \leq \frac{p c_{2}}{r^{2 \alpha}}, \quad r \geq 1
$$


Portanto,

$$
2 \lambda \int_{\mathbb{R}} \frac{d \varphi}{d r} \partial_{r} V_{2}\left|f_{n}\right|^{2} d x \geq-2 p \lambda c_{2} \int_{\mathbb{R}}\left|f_{n}\right|^{2} d x .
$$

Note que um cálculo análogo ao feito em (3.7), mas com $f_{n}$, mostra que $\partial_{t} f_{n}-\mathcal{S}_{k} f_{n}-\mathcal{A}_{2} f_{n}=$ $\partial_{t} f_{n}-i e^{\lambda \varphi} \Delta_{Z} u_{n}-i V_{2}(x, t) f_{n}$. Logo, substituindo em (3.39) e a ideia aplicada para obter (3.32), segue-se que

$$
\begin{gathered}
2 \int_{T-\frac{1}{2}}^{T+\frac{1}{2}} \int_{\mathbb{R}} \eta(t)\left|\mathcal{S}_{1} f_{n}\right|^{2} d x d t \leq 3 \int_{T-\frac{1}{2}}^{T+\frac{1}{2}} \int_{\mathbb{R}}\left|\partial_{t} f_{n}-i e^{\lambda \varphi} \Delta_{Z} u_{n}-i V_{2}(x, t) f_{n}\right|^{2} d x d t \\
+2 \lambda\left\|\varphi^{(i v)}\right\|_{\infty} \int_{T-\frac{1}{2}}^{T+\frac{1}{2}} \int_{\mathbb{R}}\left|f_{n}(x, t)\right|^{2} d x d t+\int_{\mathbb{R}}\left|f_{n}\left(x, T-\frac{1}{2}\right)\right|^{2} d x \\
+2 p \lambda c_{2} \int_{T-\frac{1}{2}}^{T+\frac{1}{2}} \int_{\mathbb{R}}\left|f_{n}(x, t)\right|^{2} d x+\int_{\mathbb{R}}\left|f_{n}\left(x, T+\frac{1}{2}\right)\right|^{2} d x-4 Z \lambda \varphi^{\prime \prime}(0) \int_{T-\frac{1}{2}}^{T+\frac{1}{2}} \eta(t)\left|f_{n}(0, t)\right|^{2} d t
\end{gathered}
$$

Agora, apaguemos o termo que envolve a constante $Z$ e seguidamente tomemos o limite quando $n \rightarrow \infty$, assim

$$
\begin{aligned}
& 2 \int_{T-\frac{1}{2}}^{T+\frac{1}{2}} \int_{\mathbb{R}} \eta(t)\left|\mathcal{S}_{1} f\right|^{2} d x d t \leq 3 \int_{T-\frac{1}{2}}^{T+\frac{1}{2}} \int_{\mathbb{R}}\left|\partial_{t} f-i e^{\lambda \varphi} \Delta_{Z} u-i V_{2}(x, t) f\right|^{2} d x d t \\
&+2 \lambda\left\|\varphi^{(i v)}\right\|_{\infty} \int_{T-\frac{1}{2}}^{T+\frac{1}{2}} \int_{\mathbb{R}}|f(x, t)|^{2} d x d t+\int_{\mathbb{R}}\left|f\left(x, T-\frac{1}{2}\right)\right|^{2} d x \\
&+2 p \lambda c_{2} \int_{T-\frac{1}{2}}^{T+\frac{1}{2}} \int_{\mathbb{R}}|f(x, t)|^{2} d x+\int_{\mathbb{R}}\left|f\left(x, T+\frac{1}{2}\right)\right|^{2} d x
\end{aligned}
$$

onde $f(x, t)=e^{\lambda \varphi} u(x, t)$. Sabemos que $u$ satisfaz a equação $\partial_{t} u-i \Delta_{Z} u-i V_{2}(x, t) u=$ $i V_{1}(x, t) u$, então usando repetidamente a hipótese (3.27), obteremos a desigualdade

$$
\int_{T-\frac{1}{2}}^{T+\frac{1}{2}} \int_{\mathbb{R}} \eta(t)\left|\mathcal{S}_{1} f\right|^{2} d x d t \leq \bar{C}_{\lambda}\left(C_{\lambda}, c_{1}, c_{2}, p, \lambda\right)
$$

Como vemos a única diferença entre (3.43) e (3.35), é que $\bar{C}_{\lambda}$ também vai depender de $c_{2}$. Portanto, também neste caso chegamos à mesma desigualdade em (3.38).

- Caso $Z<0$. A diferença deste caso em relação ao caso $Z \geq 0$, está em que o termo

$$
-4 Z \lambda \varphi^{\prime \prime}(0) \int_{T-\frac{1}{2}}^{T+\frac{1}{2}} \eta(t)|f(0, t)|^{2} d t
$$

não pode ser apagado. Assim, um procedimento análogo ao usado no caso $Z \geq 0$, mostra que

$$
\int_{T-\frac{1}{2}}^{T+\frac{1}{2}} \int_{\mathbb{R}} \eta(t)\left|\mathcal{S}_{1} f\right|^{2} d x d t \leq \bar{C}_{\lambda}-4 Z \lambda \varphi^{\prime \prime}(0) \int_{T-\frac{1}{2}}^{T+\frac{1}{2}} \eta(t)|f(0, t)|^{2} d t .
$$

Já que $\eta$ é limitada sobre o intervalo $\left[T-\frac{1}{2}, T+\frac{1}{2}\right]$ e $f(0, t)=u(0, t)$, obtemos da hipótese (3.28) uma desigualdade como a obtida em (3.35) ou (3.43), só que em ambos casos, $\bar{C}_{\lambda}$ também depende $|Z|$. Esta desigualdade, são o ponto de partida, para obter a conclusão desejada, a desigualdade (3.38). 
Lema 3.12 Se cosideramos as hipótese do Lema 3.11,

(i) para $\lambda \geq \lambda_{0}$ satisfazendo (A.14), no caso $V_{2} \equiv 0$,

ou

(ii) para $\lambda \geq \max \left\{\lambda_{3}, \lambda_{4}, \lambda_{6}\right\}$ satisfazendo (A.12), (A.13) e (A.18) no apêndice A, no caso $V_{2} \not \equiv 0$,

então, para qualquer $j \in \mathbb{Z}^{+}$

$$
\int_{T_{1}}^{T_{j}} \int_{\mathbb{R}} \frac{e^{2 \lambda \varphi(x)}|u(x, t)|^{2}}{\langle x\rangle^{4-3 p}} d x d t \leq C_{\lambda}^{\prime},
$$

uniformemente em $j \in \mathbb{Z}^{+}$.

Demonstração. Da mesma maneira que fizemos na demonstração do Lema anterior, vamos dividir a demonstração desta em vários casos.

- Caso $Z \geq 0$ e $V_{2} \equiv 0$. Seja $\left(u_{n}\right)_{n \geq 1}$ a sequência como a usada na demonstração do Lema 3.11. Logo, se aplicamos a Proposição 3.7 às funções $f_{n}(x, t)=e^{\lambda \varphi} u_{n}(x, t)$, com os operadores $\mathcal{S}_{1}$ e $\mathcal{A}_{11}$ fixados, temos

$$
\begin{gathered}
\int_{T_{1}}^{T_{j}}\left\langle\mathcal{S}_{1} f_{n}, \mathcal{A}_{11} f_{n}\right\rangle+\left\langle\mathcal{A}_{11} f_{n}, \mathcal{S}_{1} f_{n}\right\rangle d t \leq \int_{T_{1}}^{T_{j}}\left\langle\mathcal{S}_{1} f_{n}, \mathcal{A}_{11} f_{n}\right\rangle+\left\langle\mathcal{A}_{11} f_{n}, \mathcal{S}_{1} f_{n}\right\rangle d t \\
\quad+\int_{T_{1}}^{T_{j}} \int_{\mathbb{R}}\left|\mathcal{S}_{1} f_{n}\right|^{2} d x d t \\
\leq \int_{T_{1}}^{T_{j}} \int_{\mathbb{R}}\left|\partial_{t} f_{n}-\left(\mathcal{S}_{1}+\mathcal{A}_{11}\right) f_{n}\right| 2 d x d t+\left|\int_{\mathbb{R}} \mathcal{S}_{1} f_{n}\left(T_{j}\right) \overline{f_{n}\left(T_{j}\right)} d x\right|+\left|\int_{\mathbb{R}} \mathcal{S}_{1} f_{n}\left(T_{1}\right) \overline{f_{n}\left(T_{1}\right)} d x\right| \\
\leq \int_{T_{1}}^{T_{j}} \int_{\mathbb{R}}\left|\partial_{t} f_{n}-i e^{\lambda \varphi} \Delta_{Z} u_{n}\right|^{2} d x d t+\frac{1}{2} \int_{\mathbb{R}}\left(\left|\mathcal{S}_{1} f_{n}\left(T_{j}\right)\right|^{2}+\left|f_{n}\left(T_{j}\right)\right|^{2}\right) d x \\
+\frac{1}{2} \int_{\mathbb{R}}\left(\left|\mathcal{S}_{1} f_{n}\left(T_{1}\right)\right|^{2}+\left|f_{n}\left(T_{1}\right)\right|^{2}\right) d x .
\end{gathered}
$$

Agora, vamos usar a identidade (3.17) trocando $\xi$ por $f_{n}$, e substituindo-a em (3.46)

$$
\begin{aligned}
& \int_{T_{1}}^{T_{j}} \int_{\mathbb{R}}\left(4 \lambda^{3}\left(\varphi^{\prime}\right)^{2} \varphi^{\prime \prime}\left|f_{n}\right|^{2}-\lambda \varphi^{(i v)}\left|f_{n}\right|^{2}+4 \lambda \varphi^{\prime \prime}\left|\partial_{x} f_{n}\right|^{2}\right) d x d t+4 Z \lambda \varphi^{\prime \prime}(0) \int_{T_{1}}^{T_{j}}\left|f_{n}(0, t)\right|^{2} d t \\
& \leq \int_{T_{1}}^{T_{j}} \int_{\mathbb{R}}\left|\partial_{t} f_{n}-i e^{\lambda \varphi} \Delta_{Z} u_{n}\right|^{2} d x+\frac{1}{2} \int_{\mathbb{R}}\left(\left|\mathcal{S}_{1} f_{n}\left(T_{j}\right)\right|^{2}+\left|f_{n}\left(T_{j}\right)\right|^{2}\right) d x \\
& \quad+\frac{1}{2} \int_{\mathbb{R}}\left(\left|\mathcal{S}_{1} f_{n}\left(T_{1}\right)\right|^{2}+\left|f_{n}\left(T_{1}\right)\right|^{2}\right) d x d t
\end{aligned}
$$

A continuação passamos o limite quando $n \rightarrow \infty$, resultando

$$
\begin{aligned}
& \int_{T_{1}}^{T_{j}} \int_{\mathbb{R}}\left(4 \lambda^{3}\left(\varphi^{\prime}\right)^{2} \varphi^{\prime \prime}|f|^{2}-\lambda \varphi^{(i v)}|f|^{2}+4 \lambda \varphi^{\prime \prime}\left|\partial_{x} f\right|^{2}\right) d x d t+4 Z \lambda \varphi^{\prime \prime}(0) \int_{T_{1}}^{T_{j}}|f(0, t)|^{2} d t \\
& \leq \int_{T_{1}}^{T_{j}} \int_{\mathbb{R}}\left|\partial_{t} f-i e^{\lambda \varphi} \Delta_{Z} u\right|^{2} d x d t+\frac{1}{2} \int_{\mathbb{R}}\left(\left|\mathcal{S}_{1} f\left(T_{j}\right)\right|^{2}+\left|f\left(T_{j}\right)\right|^{2}\right) d x \\
& \quad+\frac{1}{2} \int_{\mathbb{R}}\left(\left|\mathcal{S}_{1} f\left(T_{1}\right)\right|^{2}+\left|f\left(T_{1}\right)\right|^{2}\right) d x \\
& \leq \int_{T_{1}}^{T_{j}} \int_{\mathbb{R}}\left|e^{\lambda \varphi(x)} V_{1}(x, t) u\right|^{2} d x d t+\bar{C}_{\lambda}
\end{aligned}
$$


onde temos usado que $u$ satisfaz a equação diferencial $\partial_{t} u-i \Delta_{Z} u=i V_{1} u$, o Lema 3.11 e a hipótese (3.27).

Devido a que $Z \geq 0$ e $\varphi^{\prime \prime}$ é positiva, obtemos

$$
\int_{T_{1}}^{T_{j}} \int_{\mathbb{R}}\left(4 \lambda^{3}\left(\varphi^{\prime}\right)^{2} \varphi^{\prime \prime}-\lambda \varphi^{(i v)}-\left|V_{1}\right|^{2}\right)|f|^{2} d x d t \leq \bar{C}_{\lambda}
$$

Agora, para $\lambda \geq \lambda_{0}$, a estimativa (A.14), implica que

$$
\begin{aligned}
\int_{T_{1}}^{T_{j}} \int_{\mathbb{R}} \frac{\lambda|f|^{2}}{\langle x\rangle^{4-3 p}} d x d t & \leq \int_{T_{1}}^{T_{j}} \int_{\mathbb{R}}\left(4 \lambda^{3}\left(\varphi^{\prime}\right)^{2} \varphi^{\prime \prime}-\lambda \varphi^{(i v)}-\left|V_{1}\right|^{2}\right)|f|^{2} d x d t \\
& \leq \bar{C}_{\lambda}
\end{aligned}
$$

- Caso $Z \geq 0$ e $V_{2} \not \equiv 0$. Para o caso geral, $V_{2} \not \equiv 0$, procedemos de maneira similar ao caso anterior, só que ao usar a Proposição 3.7 , fixamos os operadores $\mathcal{S}_{1}$ e $\mathcal{A}_{12}$, para obtermos a desigualdade análoga,

$$
\begin{aligned}
& \int_{T_{1}}^{T_{j}} \int_{\mathbb{R}}\left(4 \lambda^{3}\left(\varphi^{\prime}\right)^{2} \varphi^{\prime \prime}|f|^{2}-\lambda \varphi^{(i v)}|f|^{2}+2 \lambda \frac{d \varphi}{d r} \partial_{r} V_{2}|f|^{2}+4 \lambda \varphi^{\prime \prime}\left|\partial_{x} f\right|^{2}\right) d x d t \\
& +4 Z \lambda \varphi^{\prime \prime}(0) \int_{T_{1}}^{T_{j}}|f(0, t)|^{2} d t \leq \int_{T_{1}}^{T_{j}} \int_{\mathbb{R}}\left|e^{\lambda \varphi(x)} V_{1}(x, t) u\right|^{2} d x d t+\bar{C}_{\lambda},
\end{aligned}
$$

onde temos usado que $u$ satisfaz a equação diferencial $\partial_{t} u-i \Delta_{Z} u-i V_{2} u=i V_{1} u$.

Daí, como $Z \geq 0$ e $\varphi^{\prime \prime}$ positiva,

$$
\int_{T_{1}}^{T_{j}} \int_{\mathbb{R}}\left(4 \lambda^{3}\left(\varphi^{\prime}\right)^{2} \varphi^{\prime \prime}-\lambda \varphi^{(i v)}-\left|V_{1}\right|^{2}+2 \lambda \frac{d \varphi}{d r}\left(\partial_{r} V_{2}\right)^{-}\right)|f|^{2} d x d t \leq \bar{C}_{\lambda}
$$

mas lembre que o suporte de $V_{2}$ está contido $\{(x, t) \in|x| \geq 1\}$, logo,

$$
\begin{aligned}
& \int_{T_{1}}^{T_{j}} \int_{|x| \geq 1}\left(4 \lambda^{3}\left(\varphi^{\prime}\right)^{2} \varphi^{\prime \prime}-\lambda \varphi^{(i v)}-\left|V_{1}\right|^{2}+2 \lambda \frac{d \varphi}{d r}\left(\partial_{r} V_{2}\right)^{-}\right)|f|^{2} d x d t \\
& +\int_{T_{1}}^{T_{j}} \int_{|x| \leq 1}\left(4 \lambda^{3}\left(\varphi^{\prime}\right)^{2} \varphi^{\prime \prime}-\lambda \varphi^{(i v)}-\left|V_{1}\right|^{2}\right)|f|^{2} d x d t \leq \bar{C}_{\lambda} .
\end{aligned}
$$

Agora, para os $\lambda \geq \max \left\{\lambda_{3}, \lambda_{4}, \lambda_{6}\right\}$, as desigualdade (A.12), (A.13) e (A.18) satisfazemse simultaneamente. Portanto, usando estas desigualdades em (3.52), obtemos a mesma desigualdade que em (3.50), isto é,

$$
\int_{T_{1}}^{T_{j}} \int_{\mathbb{R}} \frac{\lambda|f|^{2}}{\langle x\rangle^{4-3 p}} d x d t \leq \bar{C}_{\lambda}
$$

- Caso $Z<0$. A diferença importante com relação aos casos anteriores é que termo $4 Z \lambda \varphi^{\prime \prime}(0) \int_{T_{1}}^{T_{j}}|f(0, t)|^{2} d t$ não pode ser apagado. Logo, seguindo as mesmas ideias obtemos

$$
\int_{T_{1}}^{T_{j}} \int_{\mathbb{R}}\left(4 \lambda^{3}\left(\varphi^{\prime}\right)^{2} \varphi^{\prime \prime}-\lambda \varphi^{(i v)}-\left|V_{1}\right|^{2}\right)|f|^{2} d x d t \leq \bar{C}_{\lambda}-4 Z \lambda \varphi^{\prime \prime}(0) \int_{T_{1}}^{T_{j}}|f(0, t)|^{2} d t
$$


e também,

$$
\begin{aligned}
\int_{T_{1}}^{T_{j}} \int_{\mathbb{R}}\left(4 \lambda^{3}\left(\varphi^{\prime}\right)^{2} \varphi^{\prime \prime}-\lambda \varphi^{(i v)}-\left|V_{1}\right|^{2}\right. & \left.+2 \lambda \frac{d \varphi}{d r}\left(\partial_{r} V_{2}\right)^{-}\right)|f|^{2} d x d t \\
& \leq \bar{C}_{\lambda}-4 Z \lambda \varphi^{\prime \prime}(0) \int_{T_{1}}^{T_{j}}|f(0, t)|^{2} d t
\end{aligned}
$$

Assim, da hipótese (3.28) conseguimos,

$$
\int_{T_{1}}^{T_{j}} \int_{\mathbb{R}}\left(4 \lambda^{3}\left(\varphi^{\prime}\right)^{2} \varphi^{\prime \prime}-\lambda \varphi^{(i v)}-\left|V_{1}\right|^{2}\right)|f|^{2} d x d t \leq \bar{C}_{\lambda}^{\prime}
$$

e também,

$$
\int_{T_{1}}^{T_{j}} \int_{\mathbb{R}}\left(4 \lambda^{3}\left(\varphi^{\prime}\right)^{2} \varphi^{\prime \prime}-\lambda \varphi^{(i v)}-\left|V_{1}\right|^{2}+2 \lambda \frac{d \varphi}{d r}\left(\partial_{r} V_{2}\right)^{-}\right)|f|^{2} d x d t \leq \bar{C}_{\lambda}^{\prime},
$$

onde $\bar{C}_{\lambda}^{\prime}$ também vai depender de $Z$. Se fixamos $\lambda \geq \lambda_{0}$ para a penúltima destas desigualdades e $\lambda \geq \max \left\{\lambda_{3}, \lambda_{4}, \lambda_{6}\right\}$ para a última, de novo obtemos

$$
\int_{T_{1}}^{T_{j}} \int_{\mathbb{R}} \frac{\lambda|f|^{2}}{\langle x\rangle^{4-3 p}} d x d t \leq \bar{C}_{\lambda}^{\prime}
$$

como se queria mostrar.

A seguir vamos estabelecer a demonstração do teorema 3.10.

Demonstração do Teorema 3.10. Inicialmente temos que, pelo Teorema 2.10, a norma $L^{2}$ da solução de (3.21) é preservada, pois o potencial $V$ é de valor real. Isto é, para todo $t \in \mathbb{R}$

$$
\|u(t)\|_{L^{2}}=\left\|u_{0}\right\|_{L 2}
$$

Agora, escolhamos $\lambda_{s}=\max \left\{\lambda_{0}, \lambda_{3}, \lambda_{4}, \lambda_{6}\right\}$, então da desigualdade (3.45), obtemos

$$
\begin{aligned}
\left(T_{j}-T_{1}\right)\left\|u_{0}\right\|_{L^{2}}^{2} & =\int_{T_{1}}^{T_{j}} \int_{\mathbb{R}}|u(x, t)|^{2} d x d t \\
& =\int_{T_{1}}^{T_{j}} \int_{\mathbb{R}}|u(x, t)|^{2} \frac{e^{2 \lambda_{s} \varphi(x)}}{\langle x\rangle^{4-3 p}}\langle x\rangle^{4-3 p} e^{-2 \lambda_{s} \varphi(x)} d x d t \\
& \leq \sup _{x \in \mathbb{R}}\left\{\langle x\rangle^{4-3 p} e^{-2 \lambda_{s} \varphi(x)}\right\} \int_{T_{1}}^{T_{j}} \int_{\mathbb{R}} \frac{|f(x, t)|^{2}}{\langle x\rangle^{4-3 p}} d x d t \leq C_{\lambda_{s}} .
\end{aligned}
$$

Agora, da forma como foi escolhida a sequência $\left\{T_{j}\right\}_{j=1}^{\infty}\left(T_{j} \rightarrow \infty\right)$, temos imediatamente que esta última desigualdade é uma contradição, exceto quando $u_{0} \equiv 0$. Portanto, $u(t)=0$, para todo $t \in \mathbb{R}$.

Devido a que $1 /\left(1+x^{2}\right)^{1 / 2} \leq c /\left(1+x^{2}\right)^{\alpha}$, com $0 \leq \alpha 1 / 2$ temos como consequência:

Corolário 3.13 Seja $u \in C\left([0, \infty): D\left(-\Delta_{Z}\right)\right) \cap C^{1}\left((0, \infty): L^{2}(\mathbb{R})\right)$ uma solução da equação (3.21) com potencial $V \in L^{\infty}(\mathbb{R} \times[0, \infty))$,

$$
|V(x, t)| \leq \frac{c_{1}}{\left(1+x^{2}\right)^{\frac{1}{2}}}
$$

e para algum $p>1$ e $\lambda>0$, 
(i)

$$
\sup _{t>0} \int_{\mathbb{R}} e^{2 M \lambda|x|^{p}}|u(x, t)|^{2} d x \leq C_{\lambda}
$$

sempre que $Z \geq 0$,

ou

(ii)

$$
\int_{0}^{\infty}|u(0, t)|^{2} d t+\sup _{t>0} \int_{\mathbb{R}} e^{2 M \lambda|x|^{p}}|u(x, t)|^{2} d x \leq C_{\lambda},
$$

sempre que $Z<0$,

onde $M>0$ é a constante dada no item 3. da Proposição 3.1, então $u \equiv 0$.

Teorema 3.14 Seja $u \in C\left([0, \infty): D\left(-\Delta_{Z}\right)\right) \cap C^{1}\left((0, \infty): L^{2}(\mathbb{R})\right)$ uma solução da equação (3.21) com potencial $V \in L^{\infty}(\mathbb{R} \times[0, \infty))$ satisfazendo

$$
V(x, t)=V_{1}(x, t)+V_{2}(x, t),
$$

com $V_{j}, j=1,2$ de valor real,

$$
\left|V_{1}(x, t)\right| \leq \frac{c_{1}}{\left(1+x^{2}\right)^{1 / 4+\epsilon_{0} / 2}}=\frac{c_{1}}{\langle x\rangle^{1 / 2+\epsilon_{0}}}, \quad \epsilon_{0}>0
$$

e $V_{2}$ com suporte em $\{(x, t):|x| \geq 1\}$ tal que

$$
-\left(\partial_{r} V_{2}(x, t)\right)^{-} \leq \frac{c_{2}}{|x|^{1+\epsilon_{0}}}, \quad a^{-}=\min \{a ; 0\} .
$$

Então, existe uma constante $\lambda_{s}=\lambda_{s}\left(\|V\|_{L^{\infty}(\mathbb{R} \times[0, \infty))} ; c_{1} ; c_{2} ; \epsilon_{0}\right)>0$ tal que,

i. se para $Z \geq 0$, temos

$$
\sup _{t>0} \int_{\mathbb{R}} e^{2 M \lambda_{s}|x|}|u(x, t)|^{2} d x \leq C_{\lambda_{s}}
$$

ou

ii. se para $Z<0$, temos

$$
\int_{0}^{\infty}|u(0, t)|^{2} d t+\sup _{t>0} \int_{\mathbb{R}} e^{2 M \lambda_{s}|x|}|u(x, t)|^{2} d x \leq C_{\lambda_{s}},
$$

onde $M>0$ é a constante dada no item 3. da Proposição 3.1, então

$$
u \equiv 0 \text {. }
$$

A demonstração deste Teorema será dividida em três lemas, dois dos quais contem as estimativas básicas para a demonstração do Teorema 3.14, como foi feito na demonstração do Teorema 3.10 .

Lema 3.15 Sejam u e V satisfazendo as condições do Teorema 3.14 e suponha $\lambda>0$ tal que

(i) se para $Z \geq 0$, temos

$$
\sup _{t>0} \int_{\mathbb{R}} e^{2 M \lambda|x|}|u(x, t)|^{2} d x \leq C_{\lambda}
$$


(ii) se para $Z<0$, temos

$$
\int_{0}^{\infty}|u(0, t)|^{2} d t+\sup _{t>0} \int_{\mathbb{R}} e^{2 M \lambda|x|}|u(x, t)|^{2} d x \leq C_{\lambda},
$$

onde $M>0$ é a constante no item 3. da Proposição 3.2. Então, existe uma sequência de números reais $\left\{T_{j}: j \in \mathbb{Z}^{+}\right\}$com $T_{j} \uparrow \infty$ quando $j \uparrow \infty$ tal que

$$
\sup _{j \in \mathbb{Z}^{+}} \int_{\mathbb{R}}\left|\mathcal{S}_{2} f\left(x, T_{j}\right)\right|^{2} d x \leq \bar{C}_{\lambda}
$$

onde $f(x, t)=e^{\lambda \psi(x)} u(x, t), \mathcal{S}_{2}$ é o operador dado em (3.8). Aqui, quando $Z \geq 0, \bar{C}_{\lambda}$ denota uma constante que depende $C_{\lambda}, \lambda,\|V\|_{L^{\infty}}, p$. Além disso, se $Z<0$, esta constante, $\bar{C}_{\lambda}$ também depende de $|Z|$.

Demonstração. A linha de argumentação na demonstração deste lema, é a mesma que a do Lema 3.11, com algumas diferenças para levar em consideração e que enumeramos a continuação:

1. Usamos a função $\psi$ construída na Proposição 3.2 .

2. A função auxiliar é $e^{\lambda \psi}$.

3. Quando aplicamos a Proposição 3.8 os operadores que fixamos são $\mathcal{S}_{2}, \mathcal{A}_{21}$ e $\mathcal{A}_{22}$ definidos respectivamente em (3.8), (3.9) e (3.10).

4. Para as funções $f_{n}(x, t)=e^{\lambda \psi} u_{n}(x, t)$, com $u_{n}$ como no Lema 3.11, usamos as identidades

$$
\partial_{t} f_{n}-i e^{\lambda \psi} \Delta_{Z} u_{n}=\partial_{t} f_{n}-\left(\mathcal{S}_{2}+\mathcal{A}_{21}\right) f_{n}
$$

e

$$
\partial_{t} f_{n}-i e^{\lambda \psi} \Delta_{Z} u_{n}-i V_{2} f_{n}=\partial_{t} f_{n}-\left(\mathcal{S}_{2}+\mathcal{A}_{22}\right) f_{n}
$$

5. Usamos as identidades (3.19) e (3.20).

6. Neste Lema $f(x, t)=e^{\lambda \psi} u(x, t)$.

Seguindo estas recomendações é só reescrever a demonstração do Lema 3.12, trocando $\varphi$ por $\psi$.

Já que a demonstração do seguinte Lema é similar à demonstração do Lema 3.12, omitiremos alguns detalhes.

Lema 3.16 Se mantermos as hipótese do Lema 3.15,

(i) para $\lambda \geq \lambda_{0}^{\prime}=\max \left\{\lambda_{2}^{\prime}, \lambda_{3}^{\prime}\right\}$ satisfazendo (A.24) e (A.25), no caso $V_{2} \equiv 0$, $o u$

(ii) para $\lambda \geq \lambda_{6}^{\prime}=\max \left\{\lambda_{0}^{\prime}, \lambda_{5}^{\prime}\right\}$ satisfazendo (A.29), no caso $V_{2} \not \equiv 0$,

então para qualquer $j \in \mathbb{Z}^{+}$

$$
\int_{T_{1}}^{T_{j}} \int_{|x| \leq 1} e^{2 \lambda \psi(x)}|u(x, t)|^{2} d x d t+\int_{T_{1}}^{T_{j}} \int_{|x| \geq 1} \frac{e^{2 \lambda \psi(x)}|u(x, t)|^{2}}{|x|(1+\log |x|)^{2}} d x d t \leq C_{\lambda}^{\prime},
$$

uniformemente em $j \in \mathbb{Z}^{+}$. 
Demonstração. • Caso $Z \geq 0$ e $V_{2} \equiv 0$. Combinando a Proposição 3.7 e a conclusão do Lema anterior e seguindo a mesma linha de argumentação usada para obter a desigualdade (3.48), conseguimos

$$
\int_{T_{0}}^{T_{1}}\left\langle\mathcal{S}_{2} f, \mathcal{A}_{21} f\right\rangle+\left\langle\mathcal{A}_{21} f, \mathcal{S}_{2} f\right\rangle d t \leq \int_{T_{1}}^{T_{j}} \int_{\mathbb{R}}\left|e^{\lambda \psi(x)} V_{1}(x, t) u\right|^{2} d x d t+\bar{C}_{\lambda},
$$

onde $f(x, t)=e^{\lambda \psi} u(x, t)$ e os operadores $\mathcal{S}_{2}$ e $\mathcal{A}_{21}$ são os definidos em (3.8) e (3.9) respectivamente.

Agora, se trocamos $\xi$ por $f_{n}(x, t)=e^{\lambda \psi} u_{n}(x, t)$ na identidade usamos a identidade (3.19) e logo passamos limite quando $n \rightarrow \infty$, resulta que essa identidade também é válida para $f$. Assim, (3.65) transforma-se em

$$
\begin{array}{r}
\int_{T_{1}}^{T_{j}} \int_{\mathbb{R}}\left(4 \lambda^{3}\left(\psi^{\prime}\right)^{2} \psi^{\prime \prime}|f|^{2}-\lambda \psi^{(i v)}|f|^{2}+4 \lambda \psi^{\prime \prime}\left|\partial_{x} f\right|^{2}\right) d x d t+4 Z \lambda \psi^{\prime \prime}(0) \int_{T_{1}}^{T_{j}}|f(0, t)|^{2} d t \\
\leq \int_{T_{1}}^{T_{j}} \int_{\mathbb{R}}\left|e^{\lambda \psi(x)} V_{1}(x, t) u\right|^{2} d x d t+\bar{C}_{\lambda} .
\end{array}
$$

Logo, para $Z \geq 0$

$$
\int_{T_{1}}^{T_{j}} \int_{\mathbb{R}}\left(4 \lambda^{3}\left(\psi^{\prime}\right)^{2} \psi^{\prime \prime}-\lambda \psi^{(i v)}-\left|V_{1}\right|^{2}\right)|f|^{2} d x d t+\int_{T_{1}}^{T_{j}} \int_{\mathbb{R}} 4 \lambda \psi^{\prime \prime}\left|\partial_{x} f\right|^{2} d x d t \leq \bar{C}_{\lambda} .
$$

Escolhamos $0<\epsilon \leq 1$ e $\lambda \geq \lambda_{0}^{\prime}$, portanto as desigualdades (A.24) e (A.25) são satisfeitas, e assim

$$
\int_{T_{1}}^{T_{j}} \int_{|x| \geq 1}\left(4 \lambda^{3}\left(\psi^{\prime}\right)^{2} \psi^{\prime \prime}-\lambda \psi^{(i v)}-\left|V_{1}\right|^{2}\right)|f|^{2} d x d t \geq \int_{T_{1}}^{T_{j}} \int_{|x| \geq 1} \frac{\lambda|f|^{2}}{|x|(1+\log |x|)^{2}} d x d t \geq 0
$$

e

$$
\int_{T_{1}}^{T_{j}} \int_{\epsilon \leq|x| \leq 1}\left(4 \lambda^{3}\left(\psi^{\prime}\right)^{2} \psi^{\prime \prime}-\lambda \psi^{(i v)}-\left|V_{1}\right|^{2}\right)|f|^{2} d x d t \geq \int_{T_{1}}^{T_{j}} \int_{\epsilon \leq|x| \leq 1} \lambda|f|^{2} d x d t \geq 0 .
$$

Agora, combinando as duas afirmações imediatamente anteriores com a desigualdade (3.67), encontramos que

$$
\begin{aligned}
& \int_{T_{1}}^{T_{j}} \int_{|x| \leq \epsilon}\left(4 \lambda^{3}\left(\psi^{\prime}\right)^{2} \psi^{\prime \prime}-\lambda \psi^{(i v)}-\left|V_{1}\right|^{2}\right)|f|^{2} d x d t+\int_{T_{1}}^{T_{j}} \int_{|x| \leq 2 \epsilon} 4 \lambda \psi^{\prime \prime}\left|\partial_{x} f\right|^{2} d x d t \\
& \leq C_{\lambda} \text {, }
\end{aligned}
$$

para os $\lambda \geq \lambda_{0}^{\prime}$. Uma pequena manipulação desta desigualdade, conseguimos

$$
4 \lambda \int_{T_{1}}^{T_{j}} \int_{|x| \leq 2 \epsilon}\left|\partial_{x} f\right|^{2} d x d t \leq \bar{C}_{\lambda}+\left(\lambda\left\|\psi^{(i v)}\right\|_{L^{\infty}}+\left\|V_{1}\right\|_{L^{\infty}, \infty}^{2}\right) \int_{T_{1}}^{T_{j}} \int_{|x| \leq \epsilon}|f|^{2} d x d t
$$

pois, como $\psi^{\prime}(0)=0$, o valor mínimo de $\left(\psi^{\prime}\right)^{2} \psi^{\prime \prime}$ é zero sobre o intervalo $[-\epsilon, \epsilon]$. Agora, escolhendo $\theta \in C^{\infty}(\mathbb{R})$ tal que $\theta(x) \equiv 1$ para $|x| \leq \epsilon, 0 \leq \theta \leq 1$ com $\operatorname{Supp}(\theta) \subset\{x$ : 
$|x| \leq 2 \epsilon\}$ e usando da desigualdade de Poincaré, obtemos para cada $t \in\left[T_{1}, T_{j}\right]$,

$$
\begin{aligned}
\int_{|x| \leq \epsilon}|f|^{2} d x & \leq \int_{|x| \leq 2 \epsilon}|\theta(x) f|^{2} d x \leq 4 \epsilon^{2} \int_{|x| \leq 2 \epsilon}\left|\partial_{x}(\theta(x) f)\right|^{2} d x \\
& \leq 4 \epsilon^{2} \int_{|x| \leq 2 \epsilon}\left|\theta^{\prime}(x) f+\theta(x) \partial_{x} f\right|^{2} d x \\
& \leq 4 \epsilon^{2}\left[\int_{|x| \leq 2 \epsilon}\left|\partial_{x} f\right|^{2} d x+C_{\theta} \int_{\epsilon \leq|x| \leq 2 \epsilon}|f|^{2} d x\right] .
\end{aligned}
$$

Misturando as estimativas (3.70) e (3.71), obtemos

$$
\begin{aligned}
{\left[1-\epsilon^{2}\left(\left\|\psi^{(i v)}\right\|_{L^{\infty}}+\frac{\left\|V_{1}\right\|_{L^{\infty, \infty}}^{2}}{\lambda}\right)\right] } & \int_{T_{1}}^{T_{j}} \int_{|x| \leq \epsilon} \lambda|f|^{2} d x d t \\
& \leq \epsilon^{2} C_{\lambda}+\epsilon^{2} C_{\theta} \int_{T_{1}}^{T_{j}} \int_{\epsilon \leq|x| \leq 1} \lambda|f|^{2} d x d t
\end{aligned}
$$

Logo, de (3.69), temos

$$
\begin{aligned}
\frac{1}{C_{\theta}}\left[\frac{1}{\epsilon^{2}}\right. & \left.-\left(\left\|\psi^{(i v)}\right\|_{L^{\infty}}+\frac{\left\|V_{1}\right\|_{L^{\infty, \infty}}^{2}}{\lambda}\right)\right] \int_{T_{1}}^{T_{j}} \int_{|x| \leq \epsilon} \lambda|f|^{2} d x d t-\frac{C_{\lambda}}{C_{\theta}} \\
& \leq \int_{T_{1}}^{T_{j}} \int_{\epsilon \leq|x| \leq 1} \lambda|f|^{2} d x d t \\
& \leq \int_{T_{1}}^{T_{j}} \int_{\epsilon \leq|x| \leq 1}\left(4 \lambda^{3}\left(\psi^{\prime}\right)^{2} \psi^{\prime \prime}-\lambda \psi^{(i v)}-\left|V_{1}\right|^{2}\right)|f|^{2} d x d t .
\end{aligned}
$$

Por outro lado, da desigualdade (3.67) e a observação (3.68), temos

$$
\begin{aligned}
\int_{T_{1}}^{T_{j}} \int_{|x| \leq \epsilon} & \left(4 \lambda^{3}\left(\psi^{\prime}\right)^{2} \psi^{\prime \prime}-\lambda \psi^{(i v)}-\left|V_{1}\right|^{2}\right)|f|^{2} d x d t \\
& +\int_{T_{1}}^{T_{j}} \int_{\epsilon \leq|x| \leq 1}\left(4 \lambda^{3}\left(\psi^{\prime}\right)^{2} \psi^{\prime \prime}-\lambda \psi^{(i v)}-\left|V_{1}\right|^{2}\right)|f|^{2} d x d t \leq \bar{C}_{\lambda}
\end{aligned}
$$

Portanto,

$$
\begin{aligned}
& \int_{T_{1}}^{T_{j}} \int_{\epsilon \leq|x| \leq 1}\left(4 \lambda^{3}\left(\psi^{\prime}\right)^{2} \psi^{\prime \prime}-\lambda \psi^{(i v)}-\left|V_{1}\right|^{2}\right)|f|^{2} d x d t \\
& \leq \bar{C}_{\lambda}+\left(\lambda\left\|\psi^{(i v)}\right\|_{L^{\infty}}+\left\|V_{1}\right\|_{L^{\infty}, \infty}^{2}\right) \int_{T_{1}}^{T_{j}} \int_{|x| \leq \epsilon}|f|^{2} d x d t .
\end{aligned}
$$

Por transitividade entre as desigualdades (3.73) e (3.74), e posteriormente simplificando, obtemos

$$
\frac{1}{C_{\theta}}\left[\frac{1}{\epsilon^{2}}-\left(1+C_{\theta}\right)\left(\left\|\psi^{(i v)}\right\|_{L^{\infty}}+\frac{\left\|V_{1}\right\|_{L^{\infty}, \infty}^{2}}{\lambda}\right)\right] \int_{T_{1}}^{T_{j}} \int_{|x| \leq \epsilon} \lambda|f|^{2} d x d t-\frac{C_{\lambda}}{C_{\theta}} \leq \bar{C}_{\lambda} .
$$

Assim, fixando $\epsilon$ suficientemente pequeno, para que a expressão dentro dos parênteses seja positiva, temos

$$
\int_{T_{1}}^{T_{j}} \int_{|x| \leq \epsilon} \lambda|f|^{2} d x d t \leq C_{\lambda}^{\prime \prime}
$$


Também, de (3.67), (3.68), da desigualdade (3.69) e de (3.76) deduzimos que

$$
\begin{aligned}
\int_{T_{1}}^{T_{j}} \int_{|x| \geq \epsilon} & \left(4 \lambda^{3}\left(\psi^{\prime}\right)^{2} \psi^{\prime \prime}-\lambda \psi^{(i v)}-\left|V_{1}\right|^{2}\right)|f|^{2} d x d t \\
& \leq \bar{C}_{\lambda}+\left(\lambda\left\|\psi^{(i v)}\right\|_{L^{\infty}}+\left\|V_{1}\right\|_{L^{\infty, \infty}}^{2}\right) \int_{T_{1}}^{T_{j}} \int_{|x| \leq \epsilon}|f|^{2} d x d t \\
& \leq \bar{C}_{\lambda}^{\prime \prime \prime}
\end{aligned}
$$

Portanto, das estimativas (3.68), (3.69), (3.76) e (3.77) obtemos o resultado desejado.

- Caso $Z \geq 0$ e $V_{2} \not \equiv 0$. Para o caso geral, $V_{2} \not \equiv 0$, procedemos de maneira similar ao caso anterior, só que ao usar a Proposição 3.7, fixamos os operadores $\mathcal{S}_{2}$ e $\mathcal{A}_{22}$, para obtermos a desigualdade análoga

$$
\begin{aligned}
& \int_{T_{1}}^{T_{j}} \int_{\mathbb{R}}\left(4 \lambda^{3}\left(\psi^{\prime}\right)^{2} \psi^{\prime \prime}|f|^{2}-\lambda \psi^{(i v)}|f|^{2}+2 \lambda \frac{d \psi}{d r} \partial_{r} V_{2}|f|^{2}+4 \lambda \psi^{\prime \prime}\left|\partial_{x} f\right|^{2}\right) d x d t \\
& +4 Z \lambda \psi^{\prime \prime}(0) \int_{T_{1}}^{T_{j}}|f(0, t)|^{2} d t \leq \int_{T_{1}}^{T_{j}} \int_{\mathbb{R}}\left|e^{\lambda \psi(x)} V_{1}(x, t) u\right|^{2} d x d t+\bar{C}_{\lambda},
\end{aligned}
$$

onde temos usado que $u$ satisfaz a equação diferencial $\partial_{t} u-i \Delta_{Z} u-i V_{2} u=i V_{1} u$.

Daí, como $Z \geq 0$,

$$
\int_{T_{1}}^{T_{j}} \int_{\mathbb{R}}\left(4 \lambda^{3}\left(\psi^{\prime}\right)^{2} \psi^{\prime \prime}-\lambda \psi^{(i v)}-\left|V_{1}\right|^{2}+2 \lambda \frac{d \psi}{d r}\left(\partial_{r} V_{2}\right)^{-}\right)|f|^{2}+4 \lambda \psi^{\prime \prime}\left|\partial_{x} f\right|^{2} d x d t \leq \bar{C}_{\lambda},
$$

mas lembre que o suporte de $V_{2}$ está contido $\{(x, t) \in|x| \geq 1\}$, logo,

$$
\begin{aligned}
& \int_{T_{1}}^{T_{j}} \int_{|x| \geq 1}\left(4 \lambda^{3}\left(\psi^{\prime}\right)^{2} \psi^{\prime \prime}-\lambda \psi^{(i v)}-\left|V_{1}\right|^{2}+2 \lambda \frac{d \psi}{d r}\left(\partial_{r} V_{2}\right)^{-}\right)|f|^{2} d x d t \\
& +\int_{T_{1}}^{T_{j}} \int_{|x| \leq 1}\left(4 \lambda^{3}\left(\psi^{\prime}\right)^{2} \psi^{\prime \prime}-\lambda \psi^{(i v)}-\left|V_{1}\right|^{2}\right)|f|^{2} d x d t \leq \bar{C}_{\lambda} .
\end{aligned}
$$

Agora, para os $\lambda \geq \lambda_{5}^{\prime}$, se satisfaz a desigualdade (A.29) e então,

$$
\begin{gathered}
\int_{T_{1}}^{T_{j}} \int_{|x| \geq 1}\left(4 \lambda^{3}\left(\psi^{\prime}\right)^{2} \psi^{\prime \prime}-\lambda \psi^{(i v)}-\left|V_{1}\right|^{2}+2 \lambda \frac{d \psi}{d r}\left(\partial_{r} V_{2}\right)^{-}\right)|f|^{2} d x d t \\
\geq \int_{T_{1}}^{T_{j}} \int_{|x| \geq 1} \frac{\lambda|f|^{2}}{|x|(1+\log |x|)^{2}} d x d t \geq 0 .
\end{gathered}
$$

Para $\epsilon>0$ suficientemente pequeno dado na demonstração do caso anterior e $\lambda \geq \lambda_{6}^{\prime}$, podemos combinar as desigualdades (3.76), (3.74) e (3.69) resultando

$$
\int_{T_{1}}^{T_{j}} \int_{|x| \leq 1} \lambda|f|^{2} d x d t \leq \bar{C}_{\lambda}
$$

Assim de (3.81) e (3.79), obtemos

$$
\int_{T_{1}}^{T_{j}} \int_{|x| \geq 1}\left(4 \lambda^{3}\left(\psi^{\prime}\right)^{2} \psi^{\prime \prime}-\lambda \psi^{(i v)}-\left|V_{1}\right|^{2}+2 \lambda \frac{d \psi}{d r}\left(\partial_{r} V_{2}\right)^{-}\right)|f|^{2} d x d t \leq \bar{C}_{\lambda} .
$$


Portanto, da combinação das desigualdades (3.82), (3.81) e (3.80) conseguimos a conclusão desejada.

- Caso $Z<0$. A diferença importante com relação aos casos anteriores é que o termo $4 Z \lambda \varphi^{\prime \prime}(0) \int_{T_{1}}^{T_{j}}|f(0, t)|^{2} d t$ não pode ser apagado. Logo, seguindo as mesmas ideias vamos obter

$$
\int_{T_{1}}^{T_{j}} \int_{\mathbb{R}}\left(4 \lambda^{3}\left(\varphi^{\prime}\right)^{2} \psi^{\prime \prime}-\lambda \psi^{(i v)}-\left|V_{1}\right|^{2}\right)|f|^{2} d x d t \leq \bar{C}_{\lambda}-4 Z \lambda \psi^{\prime \prime}(0) \int_{T_{1}}^{T_{j}}|f(0, t)|^{2} d t,
$$

e também,

$$
\begin{aligned}
\int_{T_{1}}^{T_{j}} \int_{\mathbb{R}}\left(4 \lambda^{3}\left(\psi^{\prime}\right)^{2} \psi^{\prime \prime}-\lambda \psi^{(i v)}-\left|V_{1}\right|^{2}\right. & \left.+2 \lambda \frac{d \psi}{d r}\left(\partial_{r} V_{2}\right)^{-}\right)|f|^{2} d x d t \\
& \leq \bar{C}_{\lambda}-4 Z \lambda \psi^{\prime \prime}(0) \int_{T_{1}}^{T_{j}}|f(0, t)|^{2} d t .
\end{aligned}
$$

Assim, da hipótese (3.61) conseguimos,

$$
\int_{T_{1}}^{T_{j}} \int_{\mathbb{R}}\left(4 \lambda^{3}\left(\psi^{\prime}\right)^{2} \psi^{\prime \prime}-\lambda \psi^{(i v)}-\left|V_{1}\right|^{2}\right)|f|^{2} d x d t \leq \bar{C}_{\lambda}^{\prime}
$$

e também,

$$
\int_{T_{1}}^{T_{j}} \int_{\mathbb{R}}\left(4 \lambda^{3}\left(\psi^{\prime}\right)^{2} \psi^{\prime \prime}-\lambda \psi^{(i v)}-\left|V_{1}\right|^{2}+2 \lambda \frac{d \psi}{d r}\left(\partial_{r} V_{2}\right)^{-}\right)|f|^{2} d x d t \leq \bar{C}_{\lambda}^{\prime}
$$

onde $\bar{C}_{\lambda}^{\prime}$ também vai depender de $Z$. Se fixamos $\lambda \geq \lambda_{0}^{\prime}$ para a penúltima destas desigualdades e $\lambda \geq \lambda_{6}^{\prime}$ para a última, obtemos (3.64), como se queria mostrar.

Demonstração do Teorema 3.14. Pelo Teorema 2.10, a norma $L^{2}$ da solução $u(x, t)$ de (3.21) é preservada, isto é, para todo $t \in \mathbb{R}$,

$$
\|u(\cdot, t)\|_{L^{2}}=\left\|u_{0}\right\|_{L^{2}} .
$$

Agora, para $\lambda_{s}=\max \left\{\lambda_{0}^{\prime}, \lambda_{6}^{\prime}\right\}$, podemos usar a desigualdade (3.64), para obter

$$
\begin{aligned}
\left(T_{j}-T_{1}\right)\left\|u_{0}\right\|_{L^{2}}^{2} & =\int_{T_{1}}^{T_{j}} \int_{|x| \leq 1}|u(x, t)|^{2} d x d t+\int_{T_{1}}^{T_{j}} \int_{|x| \geq 1}|u(x, t)|^{2} d x d t \\
& \leq \int_{T_{1}}^{T_{j}} \int_{|x| \leq 1} e^{2 \lambda \psi(x)}|u(x, t)|^{2} d x d t \\
& +\int_{T_{1}}^{T_{j}} \int_{|x| \geq 1}|u(x, t)|^{2} \frac{e^{2 \lambda \psi(x)}}{|x|(1+\log |x|)^{2}}|x|(1+\log |x|)^{2} e^{-2 \lambda \psi(x)} d x d t \\
& \leq \sup _{|x| \geq 1}\left\{|x|(1+\log |x|)^{2} e^{-2 \lambda \psi(x)}\right\} \int_{T_{1}}^{T_{j}} \int_{|x| \geq 1} \frac{|f(x, t)|^{2}}{|x|(1+\log |x|)^{2}} d x d t+C_{\lambda_{0}} \\
& \leq 2 C_{\lambda_{0}} .
\end{aligned}
$$

Mas da forma como foi escolhida a sequência $\left\{T_{j}\right\}_{j=1}^{\infty}\left(T_{j} \rightarrow \infty\right)$, temos que esta última desigualdade é uma contradição, exceto quando $u_{0} \equiv 0$. Portanto, $u(t)=0$, para todo $t \in \mathbb{R}$. 


\section{Capítulo 4}

\section{Aplicações da propriedades de continuação única}

Neste capítulo daremos uma aplicação das propriedades de continuação única, estudadas no capítulo anterior, no caso do conjunto das soluções que explodem em tempo finito da equação de Schrödinger não linear (chamada NLS- $\delta_{0}$ ),

$$
i \partial_{t} u+\Delta_{Z} u+\lambda|u|^{\rho-1} u=0 .
$$

Na primeira seção, estudamos a existência de soluções que explodem (blow-up) com dado inicial no domínio do operador $-\Delta_{Z}, D\left(-\Delta_{Z}\right)$, no caso $\rho \geq 5$. Na segunda seção introduzimos uma motivação do possível crescimento da explosão, usando a solução ground state e posteriormente mostramos o resultado principal deste Capítulo.

\subsection{Soluções que explodem da NLS- $\delta_{0}$}

Consideremos o problema de valor inicial,

$$
\left\{\begin{aligned}
i \partial_{t} u+\Delta_{Z} u+\lambda|u|^{\rho-1} u & =0, \quad x \in \mathbb{R}, t \in[-T, T], \rho \in \mathbb{R}^{+}, \\
u(x, 0) & =u_{0},
\end{aligned}\right.
$$

Em [1] foi estudado a boa colocação local no domínio do operador $-\Delta_{Z}$, do problema de valor inicial (4.1). Mais precisamente, foi mostrado o seguinte resultado:

Teorema 4.1 Para qualquer $u_{0} \in D\left(-\Delta_{Z}\right)$ a equação integral

$$
u(t)=e^{-i t \Delta_{Z}} u_{0}-i \lambda \int_{0}^{t} e^{-i(t-s) \Delta_{Z}}|u(s)|^{\rho-1} u(s) d s
$$

tem uma única solução $u \in C\left([-T, T] ; D\left(-\Delta_{Z}\right)\right) \cap C^{1}\left((-T, T) ; L^{2}(\mathbb{R})\right)$. Além disso, a seguinte lei de conservação mantém-se para qualquer $t \in[0, T)$ :

$$
\|u(t)\|_{L^{2}}=\left\|u_{0}\right\|_{L^{2}}
$$

Também, a boa colocação local no espaço de Sobolev $H^{1}(\mathbb{R})$, do problema de Cauchy (4.1) para o caso $\lambda>0$ é estudada em [15]. Isto é, tem-se

Teorema 4.2 Para qualquer $u_{0} \in H^{1}(\mathbb{R})$, existe $T>0$ e uma única solução $u \in C\left([0, T) ; H^{1}(\mathbb{R})\right) \cap$ $C^{1}\left([0, T) ; H^{-1}(\mathbb{R})\right)$ de $(4.1)$ tal que

$$
\lim _{t \uparrow T}\left\|\partial_{x} u\right\|_{L^{2}}=+\infty, \quad \text { se } T<+\infty
$$


Além disso, as leis de conservação de energia e carga se mantém, isto é, para cada $t \in[0, T)$ tem-se

$$
\begin{gathered}
E_{Z}(u(t))=E_{Z}\left(u_{0}\right), \\
\|u(t)\|_{L^{2}}^{2}=\left\|u_{0}\right\|_{L^{2}}^{2},
\end{gathered}
$$

onde a energia $E_{Z}$ é definida por

$$
E_{Z}(v)=\frac{1}{2}\left\|\partial_{x} v\right\|_{L^{2}}^{2}-\frac{Z}{2}|v(0)|^{2}-\frac{1}{\rho+1}\|v\|_{L^{\rho+1}}^{\rho+1}
$$

para $v \in H^{1}(\mathbb{R})$.

Também em [15], se demonstram as seguintes identidades viriais:

Proposição 4.3 Seja $u_{0} \in H^{1}(\mathbb{R})$ tal que $x u_{0} \in L^{1}(\mathbb{R})$ e u(t) a solução de (4.1) correspondente a $u_{0}$. Então, a função $F: t \mapsto\|x u(t)\|_{L^{2}}^{2}$ é de classe $C^{2} e$

$$
\begin{gathered}
\partial_{t} F(t)=4 \operatorname{Im} \int_{\mathbb{R}} x \bar{u} \partial_{x} u d x, \\
\partial_{t}^{2} F(t)=8 R_{Z}(u(t)),
\end{gathered}
$$

onde $R_{Z}$ é definido para $v \in H^{1}(\mathbb{R})$ por

$$
R_{Z}(v)=\left\|\partial_{x} v\right\|_{L^{2}}^{2}-\frac{Z}{2}|v(0)|^{2}-\frac{\rho-1}{2(\rho+1)}\|v\|_{L^{\rho+1}}^{\rho+1} .
$$

Assim, com ajuda da proposição anteriores podemos mostrar que para certos dados iniciais do problema (4.1) existem soluções que explodem em tempo finito.

Teorema 4.4 Suponha que $\rho \geq 5$ e sejam $Z \in \mathbb{R}$ e $u_{0} \in H^{1}(\mathbb{R})$ tais que

$$
|x| u_{0} \in L^{2}(\mathbb{R}) \quad e \quad 2 E_{Z}\left(u_{0}\right)+\frac{Z}{2}\left|u_{0}(0)\right|^{2}<0 .
$$

Então, a solução u de (4.1) correspondente a $u_{0}$ explode em tempo finito, isto é, $T^{\max }<+\infty$.

Cabe notar que em [15] foi obtido um resultado de blow-up no caso $Z<0$ e $E_{Z}\left(u_{0}\right)<0$; mas da desigualdade em (4.4), podemos observar que esse resultado vale também para energia positiva, sempre que $Z$ tem que ser negativa. Também, vale se $Z$ é positiva, neste caso a energia tem que ser negativa, com o objetivo que a expressão $2 E_{Z}\left(u_{0}\right)+\frac{Z}{2}\left|u_{0}(0)\right|^{2}<0$.

Demonstração do Teorema 4.4. Primeiro, observemos que

$$
R_{Z}(u(t))=2 E_{Z}(u(t))+\frac{Z}{2}\left|u_{0}(0)\right|^{2}+\frac{5-\rho}{2(\rho+1)}\|u(t)\|_{\rho+1}^{\rho+1} .
$$

Já que $\rho \geq 5$, temos da lei de conservação de energia

$$
R_{Z}(u(t)) \leq 2 E_{Z}(u(t))+\frac{Z}{2}\left|u_{0}(0)\right|^{2}=2 E_{Z}\left(u_{0}\right)+\frac{Z}{2}\left|u_{0}(0)\right|^{2}<0,
$$

para todo $t \in\left(0, T^{\max }\right)$. 
Portanto, pela Proposição 4.3, temos

$$
\frac{d^{2}}{d t^{2}}\|x u(t)\|_{L^{2}}^{2} \leq 16 E_{Z}\left(u_{0}\right)+4 Z\left|u_{0}(0)\right|^{2},
$$

para todo $t \in\left(0, T^{\max }\right)$. Integrando duas vezes respeito ao tempo conseguimos

$$
\|x u(t)\|_{L^{2}}^{2} \leq\left(8 E_{Z}\left(u_{0}\right)+4 Z\left|u_{0}(0)\right|^{2}\right) t^{2}+4\left(\operatorname{Im} \int_{\mathbb{R}} x \bar{u}_{0} \partial_{x} u_{0} d x\right) t+\left\|x u_{0}\right\|_{L^{2}}^{2} .
$$

O membro do lado direito de (4.5) é um polinômio de ordem dois com coeficiente principal negativo. Por conseguinte, para $|t|$ grande, essa expressão torna-se negativa, que contraria o fato de $\|x u(t)\|_{L^{2}}^{2} \geq 0$. Isto implica que $T^{\max }<+\infty$.

Corolário 4.5 Se $u_{0} \in D\left(-\Delta_{Z}\right)$ satisfaz as condições do Teorema 4.4, então a solução local garantida pelo Teorema 4.1, explode em tempo finito.

\subsection{Concentração das soluções para a equação 4.1}

Foi mostrado em [10] e em [11] que para $Z \neq 0$, a equação de Schrödinger

$$
i \partial_{t} u(x, t)=-\Delta_{Z} u-|u|^{\rho-1} u,
$$

possui soluções do tipo "standing-wave" da forma $u_{s}(x, t)=e^{i \omega t} \varphi_{\omega, Z}(x)$, com

$$
\varphi_{\omega, Z}(x)=\left[\frac{(\rho+1) \omega}{2} \operatorname{sech}^{2}\left(\frac{(\rho-1) \sqrt{\omega}}{2}|x|+\tanh ^{-1}\left(\frac{Z}{2 \sqrt{\omega}}\right)\right)\right]^{\frac{1}{\rho-1}},
$$

onde $\omega>\frac{Z^{2}}{4}$ e $\varphi_{\omega, Z} \in D\left(-\Delta_{Z}\right)$.

Observemos que se $Z \geq 0$, a quantidade $\tanh ^{-1}\left(\frac{Z}{2 \sqrt{\omega}}\right)$ é positiva e assim obtemos a seguinte estimativa para as soluções do tipo standing-wave,

$$
\begin{aligned}
\left|u_{s}(x, t)\right|=\varphi_{\omega, Z}(x) & \leq\left(\frac{(\rho+1) \omega}{2}\right)^{\frac{1}{\rho-1}} e^{-\sqrt{\omega}|x|-\frac{2}{\rho-1} \tanh ^{-1}\left(\frac{Z}{2 \sqrt{\omega}}\right)} \\
& =c(a, \omega, Z) e^{-\sqrt{\omega}|x|},
\end{aligned}
$$

para todo $x, t \in \mathbb{R}$. Agora, se fixamos $Z<0$, então $\tanh ^{-1}\left(\frac{Z}{2 \sqrt{\omega}}\right)$ é negativa e assim

$$
\left|u_{s}(x, t)\right|=\varphi_{\omega, Z}(x) \leq c(\rho, \omega, Z) e^{-\sqrt{\omega}|x|},
$$

para cada $x$ no conjunto $\left\{x \in \mathbb{R}:|x|>\frac{2}{\sqrt{\omega}(\rho-1)}\left|\tanh ^{-1}\left(\frac{Z}{2 \sqrt{\omega}}\right)\right|\right\}$ e todo $t \in \mathbb{R}$. Isto é, em geral para $Z \in \mathbb{R}$ e $t \in \mathbb{R}$, a solução standing-wave, $u_{s}(x, t)$ tem decaimento exponencial da forma $e^{-\sqrt{\omega}|x|}$.

Por outro lado, aplicando a transformação pseudo conforme (ver [13]) á solução $u=u(x, t)$ de (4.6), obtemos que a função

$$
v(x, t)=\frac{e^{i \rho|x|^{2} / 4(\nu+\sigma t)}}{(\nu+\sigma t)^{1 / 2}} u\left(\frac{x}{\nu+\sigma t}, \frac{\gamma+\theta t}{\nu+\sigma t}\right), \quad \operatorname{com} \quad \nu \theta-\sigma \gamma=1
$$

satisfaz formalmente a equação

$$
i \partial_{t} v=-\Delta_{\frac{z}{\nu+\sigma t}} v-(\nu+\sigma t)^{\left(\frac{\rho}{2}-\frac{5}{2}\right)}|v|^{\rho-1} v
$$


onde $v(t) \in D\left(-\Delta_{\frac{Z}{\nu+\sigma t}}\right)$.

Em particular para a solução standing-wave, se tomamos $Z=0, \rho=5, \nu=\gamma=1, \sigma=-1$ e $\theta=0$, segue-se que

$$
v(x, t)=\frac{e^{-i t(1-t)} e^{-i x^{2} / 4(1-t)}}{(1-t)^{1 / 2}} \varphi_{\omega, 0}\left(\frac{x}{1-t}\right), \quad \omega>0,
$$

é uma solução de

$$
i \partial_{t} u(x, t)=-\Delta u-|u|^{4} u,
$$

que explode no tempo $t=1$, isto é,

$$
\lim _{t \uparrow 1}\|\nabla v(\cdot, t)\|_{2}=\infty,
$$

e no sentido das distribuições podemos ver que,

$$
\lim _{t \uparrow 1}|v(\cdot, t)|^{2}=c \delta(\cdot),
$$

onde $c$ é uma constante específica.

É claro de (4.7) que o perfil $\varphi_{\omega, 0}$ tem decaimento exponencial, isto é,

$$
\varphi_{\omega, 0}(x) \sim b_{1} e^{-b_{2}|x|}, \quad b_{1}, b_{2}>0 .
$$

Logo, a solução que explode $v(x, t)$ dada em (4.10) satisfaz

$$
|v(x, t)| \leq \frac{1}{(1-t)^{1 / 2}} Q\left(\frac{x}{1-t}\right)
$$

com $Q(x)=b_{1} e^{-b_{2}|x|}$. Assim, podemos, portanto, pergunta-nos se é possível ter um perfil de concentração mais rápido na solução de (4.11) com $\rho=5$ que o descrito em (4.15). Em outras palavras, consideremos a função $Q$ na forma

$$
Q(x)=b_{1} e^{-b_{2}|x|^{p}}, \quad b_{1}, b_{2}>0, \quad p>1,
$$

ou da forma,

$$
Q(x)=b_{1} e^{-b_{3}|x|},
$$

com $b_{3}$ suficientemente grande. Será possível que para $\rho>5$ e $Z \in \mathbb{R}$, podemos ter que a solução de (4.6) satisfaça

$$
|v(x, t)| \leq \frac{1}{(1-t)^{2 /(\rho-1)}} Q\left(\frac{x}{1-t}\right), \quad \forall x \in \mathbb{R} \quad \text { e } \quad t \in(-1,1),
$$

com $Q$ dado em (4.16) ou em (4.17).

Teorema 4.6 Seja $\rho>5$. Considere $u \in C\left((-1,1): D\left(-\Delta_{Z}\right)\right)$ uma solução da equação (4.6) satisfazendo (4.18) para $Q$ como em (4.16) para algum $p>1$ e $b_{2}>0$, ou como em (4.17) para $b_{3}>0$ suficientemente grande. Então $u \equiv 0$.

Demonstração. Se em particular tomamos $\nu=\sigma=\theta=1$ e $\gamma=0$ em (4.8), obtemos a nova função

$$
v(x, t)=\frac{1}{(1+t)^{1 / 2}} e^{i|x|^{2} / 4(1+t)} u\left(\frac{x}{1+t}, \frac{t}{1+t}\right) .
$$


Pelo Teorema 4.1 temos que $u$ satisfaz a equação (2.1), ou seja, que resolve a equação, para q.t.p $x$,

$$
i \partial_{t} u+\partial_{x}^{2} u+|u|^{\rho-1} u=0, \quad x \neq 0,
$$

$\operatorname{logo} v$ resolve a equação, para $t \in[0, \infty)$,

$$
i \partial_{t} v+\partial_{x}^{2} v+(1+t)^{\left(\frac{a}{2}-\frac{5}{2}\right)}|v|^{\rho-1} v=0, \quad x \neq 0,
$$

e $v(x, t)$ satisfazendo para $t$ fixo,

$$
\partial_{x} v\left(0^{+}, t\right)-\partial_{x} v\left(0^{-}, t\right)=\frac{Z}{1+t} v(0, t)
$$

Observamos que $v(t) \in D\left(-\Delta_{\frac{Z}{1+t}}\right)$, para todo $t \geq 0$. Da hipótese (4.18) segue-se que a solução $v(x, t)$ satisfaz

$$
\begin{aligned}
|v(x, t)| & =\frac{1}{(1+t)^{1 / 2}}\left|u\left(\frac{x}{1+t}, \frac{t}{1+t}\right)\right| \\
& \leq \frac{1}{(1+t)^{1 / 2}} \frac{1}{(1-t /(1+t))^{2 /(\rho-1)}} Q\left(\frac{\frac{x}{1+t}}{1-\frac{1}{1+t}}\right) \\
& =\frac{1}{(1+t)^{\frac{1}{2}-\frac{2}{(\rho-1)}}} Q(x) .
\end{aligned}
$$

A seguir, se consideramos o potencial $V(x, t)$ da forma

$$
V(x, t)=(1+t)^{\frac{\rho}{2}-\frac{5}{2}}|v(x, t)|^{\rho-1},
$$

temos a seguinte estimativa

$$
|V(x, t)| \leq(1+t)^{\frac{\rho}{2}-\frac{5}{2}}\left(\frac{1}{(1+t)^{\frac{1}{2}-\frac{2}{\rho-1}}}\right)^{\rho-1} Q^{\rho-1}(x)=Q^{\rho-1}(x) .
$$

Agora $Q^{\rho-1}$, já seja como em (4.16) ou como em (4.17) pode ser dominada por uma função do tipo $c /\left(1+x^{2}\right)^{\alpha / 2}$, com $c, \alpha>0$. Segue-se que o potencial em (4.21) satisfaz as hipóteses nos Teoremas 3.10 e 3.14 com $V_{2} \equiv 0$. Também do fato que $\frac{1}{2}-\frac{2}{\rho-1} \geq 0$, temos de (4.23),

$$
|v(x, t)| \leq Q(x)
$$

para todo tempo $t \geq 0$. Assim, por exemplo se $Q$ é como em (4.16), então

$$
\int_{\mathbb{R}}|v(x, t)|^{2} e^{2 \lambda|x|^{p}} d x \leq b_{1}^{2} \int_{\mathbb{R}} e^{2\left(\lambda-b_{2}\right)|x|^{p}} d x<+\infty
$$

sempre que $\lambda<b_{2}$. Analogamente, se $Q$ é como em (4.17), obtemos que $v \in L^{2}\left(e^{2 \lambda|x|} d x\right)$, sempre que $\lambda<b_{3}$.

Agora, temos que a solução $u$ satisfaz $\left\|u\left(t^{\prime}\right)\right\|_{L^{2}}=\left\|u_{0}\right\|_{L^{2}}$ para todo $t^{\prime} \in[0,1)$, assim $\|v(t)\|_{L^{2}}=\left\|v_{0}\right\|_{L^{2}}$, para todo $t \in[0, \infty)$.

Como por hipótese $u$ é dominada pela função $\frac{1}{(1-t)^{2 /(\rho-1)}} Q\left(\frac{x}{1-t}\right)$, para todo $x \in \mathbb{R}$ e $t \in$ $(-1,1)$, e $\rho>5$, obtemos

$$
\int_{0}^{\infty} \frac{1}{(1+t)^{2}}\left|u\left(0, \frac{t}{1+t}\right)\right|^{2} d t=\int_{0}^{1}|u(0, t)|^{2} d t \leq \int_{0}^{1} \frac{1}{(1-t)^{4 /(\rho-1)}} d t<+\infty .
$$


Então, da definição de $v$, temos

$$
\int_{0}^{\infty} \frac{1}{(1+t)}|v(0, t)|^{2} d t<\infty
$$

Note que nos Teoremas 3.10 e 3.14, o salto na derivada das funções $u(x, t)$ tem $Z$ fixo quando o tempo evolui e dito salto dá origem ao termo

$$
4 Z c \int_{T_{1}}^{T_{j}}|u(0, t)|^{2} d t
$$

onde $c>0$. No caso $Z \geq 0$, pudemos apagar este termo e continuar a demonstração . No caso $Z<0$, este termo é controlado com a hipótese $\int_{0}^{\infty}|u(0, t)|^{2} d t<\infty$. Agora, para a função $v$ dada em (4.19), seguindo a mesma técnica usada nas demonstrações dos Teoremas 3.10 e 3.14 , obtemos o termo

$$
4 c Z \int_{T_{1}}^{T_{j}} \frac{1}{(1+t)}|v(0, t)|^{2} d t
$$

porque o salto evolui de acordo a (4.22). Por este motivo, foi mostrado (4.25). Assim, se $Z \geq 0$, esse termo é apagado, mas se $Z<0$ esse termo é controlado por (4.25). Portanto, existem $\lambda^{\prime}$ s que mostram que $v \equiv 0$, o que implica que $u \equiv 0$. 


\section{Capítulo 5}

\section{Estudos futuros}

Neste capítulo, nosso objetivo principal será apresentar algumas idéias dos problemas que podem ser abordados em um futuro próximo.

1. Acreditamos que podemos obter condições suficientes sobre o comportamento da solução $u$ em dois tempos diferentes $t_{0}=0$ e $t_{1}=1$, da equações de Schrodinger linear com ponto de interação

$$
i \partial_{t} u(x, t)+\Delta_{Z} u(x, t)=V(x, t) u(x, t), \quad(x, t) \in \mathbb{R} \times \mathbb{R},
$$

para garantir que $u \equiv 0$ seja sua única solução.

No caso da equação não linear com ponto de interação do tipo,

$$
i \partial_{t} u(x, t)+\Delta_{Z} u(x, t)+F(u, \bar{u})=0, \quad(x, t) \in \mathbb{R} \times \mathbb{R},
$$

acreditamos que podemos mostrar a unicidade das soluções estudando o comportamento da diferença de duas soluções possíveis em dois tempos diferentes.

Em particular o caso $Z=0$, é resolvido resolvido [9] por Escauriaza, Kenig, Ponce e Vega.

2. Outros problemas que pode ser abordado é encontrar propriedades de continuação única, análogas as estudadas nesta tese ou as propostas no item anterior, para as soluções das equações do tipo Schrödinger com dois ponto de interação, a saber,

$$
i \partial_{t} u(x, t)+Z\left(\delta_{-c}+\delta_{c}\right) u(x, t)=V(x, t) u(x, t), \quad(x, t) \in \mathbb{R} \times \mathbb{R},
$$

e também,

$$
i \partial_{t} u(x, t)+Z\left(\delta_{-c}+\delta_{c}\right) u(x, t)+F(u, \bar{u})=0, \quad(x, t) \in \mathbb{R} \times \mathbb{R},
$$

onde $Z \in \mathbb{R}$ e $c>0$ são constantes.

3. Também podemos estudar propriedades de continuação única para as soluções das equações

$$
i \partial_{t} u(x, t)+Z \delta_{0}^{\prime} u(x, t)=V(x, t) u(x, t), \quad(x, t) \in \mathbb{R} \times \mathbb{R},
$$

e também

$$
i \partial_{t} u(x, t)+Z \delta_{0}^{\prime} u(x, t)+F(u, \bar{u})=0, \quad(x, t) \in \mathbb{R} \times \mathbb{R},
$$




\section{Apêndice A}

\section{Desigualdades em relação as funções $\varphi \mathrm{e}$ $\psi$}

Neste apêndice damos varias desigualdades básicas que serão usadas no capítulo 3. Na seção A.1 mostramos que para $\lambda>0$ suficientemente grande são válidas as estimativas

$$
4 \lambda^{3}\left(\varphi^{\prime}\right)^{2} \varphi^{\prime \prime}-\varphi^{(i v)} \lambda-\left|V_{1}\right|^{2} \geq \frac{\lambda}{\langle x\rangle^{4-3 p}},
$$

e

$$
4 \lambda^{3}\left(\varphi^{\prime}\right)^{2} \varphi^{\prime \prime}-\lambda \varphi^{(i v)}-\left|V_{1}\right|^{2}+2 \lambda \frac{d \varphi}{d r}\left(\partial_{r} V_{2}(x, t)\right)^{-} \geq \frac{\lambda}{\langle x\rangle^{4-3 p}} .
$$

Similarmente, na seção A.2, mostramos que para $\lambda>0$ suficientemente grande temos

$$
\begin{gathered}
4 \lambda^{3}\left(\psi^{\prime}\right)^{2} \psi^{\prime \prime}-\lambda \psi^{(i v)}-\left|V_{1}\right|^{2} \geq \frac{\lambda}{|x|(1+\log |x|)^{2}}, \quad|x| \geq \epsilon, \\
4 \lambda^{3}\left(\psi^{\prime}\right)^{2} \psi^{\prime \prime}-\lambda \psi^{(i v)}-\left|V_{1}\right|^{2} \geq \lambda, \quad \epsilon \leq|x| \leq 1,
\end{gathered}
$$

para $\epsilon>0$ pequeno, e também

$$
4 \lambda^{3}\left(\psi^{\prime}\right)^{2} \psi^{\prime \prime}-\lambda \psi^{(i v)}-\left|V_{1}\right|^{2}+2 \lambda \frac{d \psi}{d r}\left(\partial_{r} V_{2}(x, t)\right)^{-} \geq \frac{\lambda}{|x|(1+\log |x|)^{2}}, \quad|x| \geq 1 .
$$

\section{A.1 Desigualdades em relação a $\varphi$}

Devido às características de $\varphi$ a continuação vamos fazer algumas observações importantes, que ajudarão na demonstração do Lema 3.12. De fato, já que $4-3 p>0$ para qualquer $x \in \mathbb{R},|x|^{4-3 p} \leq\left|x^{2}+1\right|^{\frac{4-3 p}{2}}=\langle x\rangle^{4-3 p}$, assim

$$
\frac{1}{\langle x\rangle^{4-3 p}} \leq \frac{1}{|x|^{4-3 p}}, \quad \forall x \in \mathbb{R} \backslash\{0\} .
$$

Por outro lado, para $|x| \geq 1$,

$$
\left(\varphi^{\prime}(x)\right)^{2}=p^{2}|x|^{2(p-1)}, \quad \varphi^{\prime \prime}(x)=p(p-1)|x|^{p-2} .
$$

Logo, usando (A.1) temos

$$
\left(\varphi^{\prime}(x)\right)^{2} \varphi^{\prime \prime}(x)=p^{3}(p-1)|x|^{3 p-4} \geq \frac{c_{p}}{\langle x\rangle^{4-3 p}}, \quad|x| \geq 1,
$$


$\operatorname{com} c_{p}=p^{3}(p-1)$.

Como $p \in(1,4 / 3]$ obtemos

$$
\lim _{|x| \rightarrow \infty} \frac{\left(1+x^{2}\right)^{(4-3 p) / 2}}{|x|^{4-p}}=0
$$

portanto,

$$
\varphi^{(i v)}(x)=p(p-1)(p-2)(p-3)|x|^{p-4} \leq \frac{c_{p}^{\prime}}{\langle x\rangle^{4-3 p}},
$$

para $|x| \geq 1$ e $c_{p}^{\prime}=p(p-1)(p-2)(p-3)>0$. Por outro lado, na demonstração da Proposição anterior se vê que $\varphi^{(i v)}(0)=\frac{3}{8}(p-8)(p-6)(p-2) p<0$ e $-35 / 2<\varphi^{(i v)}(0)<-70 / 9$, quando $p \in(1,4 / 3]$. Então, escolha um $\epsilon \in(0,1)$ tal que $\varphi^{(i v)}(x)<0$ para todo $x \in(-\epsilon, \epsilon) \mathrm{e}$ também que $C_{\varphi, \epsilon}^{\prime \prime}=\min _{x \in(-\epsilon, \epsilon)}\left\{-\varphi^{(i v)}(x)\right\}>1$. No momento, consideremos o conjunto $\{x: \epsilon \leq|x| \leq 1\}$. Da continuidade das funções $\varphi^{\prime}, \varphi^{\prime \prime}$ e $\varphi^{(i v)}$ neste conjunto, o qual é compacto, obtemos

$$
\left(\varphi^{\prime}(x)\right)^{2} \varphi^{\prime \prime}(x) \geq C_{\varphi, \epsilon}=\min _{\epsilon \leq|x| \leq 1}\left\{\left(\varphi^{\prime}(x)\right)^{2} \varphi^{\prime \prime}(x)\right\}
$$

o qual é diferente de zero, pois tanto $\varphi^{\prime} \operatorname{como} \varphi^{\prime \prime}$ não se anulam no intervalo $(0,1)$. Também

$$
\left|\varphi^{(i v)}(x)\right| \leq C_{\varphi, \epsilon}^{\prime}=\max _{\epsilon \leq|x| \leq 1}\left\{\varphi^{(i v)}(x)\right\} .
$$

Agora, consideremos uma função de valor real $V \in L^{\infty}(\mathbb{R} \times[0, \infty))$ satisfazendo

$$
\left|V_{1}(x, t)\right| \leq \frac{c_{1}}{\langle x\rangle^{\alpha}}, \quad 0 \leq \alpha<1 / 2
$$

onde $\langle x\rangle=\left(1+x^{2}\right)^{1 / 2}$. Então, para $\lambda>0$, as desigualdades (A.2), (A.3)

$$
4 \lambda^{3}\left(\varphi^{\prime}\right)^{2} \varphi^{\prime \prime}-\lambda \varphi^{(i v)} \geq \frac{4 \lambda^{3} c_{p}-\lambda c_{p}^{\prime}}{\langle x\rangle^{4-3 p}}
$$

Logo, para todo $\lambda \geq \lambda_{1}=\max \left\{1, \sqrt{c_{p}^{\prime} /\left(4 c_{p}\right)}\right\}$, obtém a seguinte estimativa

$$
4 \lambda^{3}\left(\varphi^{\prime}\right)^{2} \varphi^{\prime \prime}-\lambda \varphi^{(i v)} \geq \frac{4 \lambda^{2} c_{p}-c_{p}^{\prime}}{\langle x\rangle^{4-3 p}} .
$$

Agora, adicionando a hipótese sobre $V_{1}$, conseguimos a seguinte desigualdade

$$
4 \lambda^{3}\left(\varphi^{\prime}\right)^{2} \varphi^{\prime \prime}-\lambda \varphi^{(i v)}-\left|V_{1}\right|^{2} \geq \frac{4 \lambda^{2} c_{p}-c_{p}^{\prime}-c_{1}}{\langle x\rangle^{4-3 p}} .
$$

Já que o polinômio de grau $2, m(\lambda)=4 c_{p} \lambda^{2}-\lambda-\left(c_{p}^{\prime}+c_{1}\right)$, tem coeficiente principal positivo, para cada $\lambda \geq \lambda_{2}=\max \left\{\lambda_{1}, \frac{1+\sqrt{1+16 c_{p}\left(c_{p}^{\prime}+c_{1}\right)}}{8 c_{p}}\right\}, m(\lambda)>0$, de modo que

$$
4 \lambda^{3}\left(\varphi^{\prime}\right)^{2} \varphi^{\prime \prime}-\varphi^{(i v)} \lambda-\left|V_{1}\right|^{2} \geq \frac{\lambda}{\langle x\rangle^{4-3 p}},
$$

para $|x| \geq 1$ e $\lambda \geq \lambda_{2}$. 
Por outro lado, para $\epsilon \in(0,1)$ dado anteriormente, de (A.4) e (A.5) temos

$$
4 \lambda^{3}\left(\varphi^{\prime}\right)^{2} \varphi^{\prime \prime}-\varphi^{(i v)} \lambda \geq 4 C_{\varphi, \epsilon} \lambda^{3}-C_{\varphi, \epsilon}^{\prime} \lambda .
$$

Um procedimento análogo ao usado na obtenção da desigualdade (A.10) mostra que para cada $\lambda>\lambda_{3}=\max \left\{1, \frac{1+\sqrt{1+16 C_{\varphi, \epsilon}\left(C_{\varphi, \epsilon}^{\prime}+c_{1}\right)}}{8 C_{\varphi, \epsilon}}, \sqrt{C_{\varphi, \epsilon}^{\prime} /\left(4 C_{\varphi, \epsilon}\right)}\right\}$ se verifica que

$$
4 \lambda^{3}\left(\varphi^{\prime}\right)^{2} \varphi^{\prime \prime}-\lambda \varphi^{(i v)}-\left|V_{1}\right|^{2} \geq \lambda
$$

sobre o conjunto $x$ que pertencem a $\{x: \epsilon \leq|x| \leq 1\}$.

Também para $x \in(-\epsilon, \epsilon)$ e $\lambda \geq \lambda_{4}=\frac{c_{1}}{C_{\varphi, \epsilon}^{\prime \prime}-1}$, temos

$$
4 \lambda^{3}\left(\varphi^{\prime}\right)^{2} \varphi^{\prime \prime}-\lambda \varphi^{(i v)}-\left|V_{1}\right|^{2} \geq C_{\varphi, \epsilon}^{\prime \prime} \lambda-c_{1} \geq \lambda
$$

pois o valor mínimo nesse intervalo da derivada $\varphi^{\prime}$ é zero e $C_{\varphi, \epsilon}^{\prime \prime}>1$, Logo, escolhendo $\lambda_{0}=$ $\max \left\{\lambda_{1} ; \lambda_{2} ; \lambda_{3} ; \lambda_{4}\right\}$, as desigualdades (A.10), (A.12) e (A.13) satisfazem-se simultaneamente para os $\lambda \geq \lambda_{0}$. Em resumo, e do fato que $\langle x\rangle^{4-3 p} \geq 1$, para todo $x \in \mathbb{R}$ a estimativa

$$
4 \lambda^{3}\left(\varphi^{\prime}\right)^{2} \varphi^{\prime \prime}-\varphi^{(i v)} \lambda-\left|V_{1}\right|^{2} \geq \frac{\lambda}{\langle x\rangle^{4-3 p}},
$$

é válida para cada $\lambda \geq \lambda_{0}$ e $x \in \mathbb{R}$.

Se além, temos uma função $V_{2}$ cujo suporte contido no conjunto $\{(x, t):|x| \geq 1\}$ e a derivada radial, isto é, com respeito a $r=|x|$, verifica

$$
-\left(\partial_{r} V_{2}(x, t)\right)^{-} \leq \frac{c_{2}}{|x|^{(1+4 \alpha) / 3}}, \quad a^{-}=\min \{a ; 0\} .
$$

Então

$$
-\frac{d \varphi}{d r}\left(\partial_{r} V_{2}(x, t)\right)^{-} \leq \frac{(\sqrt{2})^{2 \alpha} c_{2}}{\langle x\rangle^{2 \alpha}}, \quad|x| \geq 1 .
$$

Assim, para $\lambda_{5}=\max \left\{1, \sqrt{\frac{c_{p}^{\prime}+2(\sqrt{2})^{2 \alpha} c_{2}}{4 c_{p}}}\right\}$ e $|x| \geq 1$, temos

$$
4 \lambda^{3}\left(\varphi^{\prime}\right)^{2} \varphi^{\prime \prime}-\lambda \varphi^{(i v)}-\left|V_{1}\right|^{2}+2 \lambda \frac{d \varphi}{d r}\left(\partial_{r} V_{2}(x, t)\right)^{-} \geq \frac{4 \lambda^{2} c_{p}-c_{p}^{\prime}-2(\sqrt{2})^{2 \alpha} c_{2}-c_{1}}{\langle x\rangle^{4-3 p}}
$$

Logo,

$$
4 \lambda^{3}\left(\varphi^{\prime}\right)^{2} \varphi^{\prime \prime}-\lambda \varphi^{(i v)}-\left|V_{1}\right|^{2}+2 \lambda \frac{d \varphi}{d r}\left(\partial_{r} V_{2}(x, t)\right)^{-} \geq \frac{\lambda}{\langle x\rangle^{4-3 p}}
$$

sempre que $\lambda \geq \lambda_{6}=\max \left\{\lambda_{5}, \frac{1+\sqrt{1+16 c_{p}\left(c_{p}^{\prime}+2(\sqrt{2})^{2 \alpha} c_{2}+c_{1}\right)}}{4 c_{p}}\right\}$ e $|x| \geq 1$.

\section{A.2 Desigualdades em relação a $\psi$}

Agora, vamos aproveitar as características da função $\psi$ construída na proposição anterior, e os resultados obtidos serão usados na demonstração do Lema 3.16. O primeiro que notaremos é que para $r=|x| \geq 1$,

$$
\psi^{(i v)}(r)=\frac{14+2 \log r(5+\log r)}{r^{3}(1+\log r)^{4}} \leq \frac{14+5 e^{-1}}{r(1+\log r)^{2}},
$$


o segundo que notaremos também para $r \geq 1$, é que

$$
\left(\psi^{\prime}(r)\right)^{2} \psi^{\prime \prime}(r)=\left(3-\frac{1}{\log r}\right)^{2} \frac{1}{r(1+\log r)^{2}} \geq \frac{4}{r(1+\log r)^{2}} .
$$

Então, para $\lambda \geq \lambda_{1}^{\prime}=\sqrt{\frac{14+5 e^{-1}}{16}}$, de (A.19) e (A.20) obtemos que

$$
4 \lambda^{3}\left(\psi^{\prime}(r)\right)^{2} \psi^{\prime \prime}(r)-\lambda \psi^{(i v)}(r) \geq \frac{16 \lambda^{2}-\left(14+5 e^{-1}\right)}{r(1+\log r)^{2}},
$$

para $r \geq 1$. Logo, se supomos uma função de valor real $V_{1} \in L^{\infty}(\mathbb{R} \times[0, \infty))$ tal que

$$
\left|V_{1}(x, t)\right| \leq \frac{c_{1}}{\langle x\rangle^{\frac{1}{2}+\epsilon_{0}}}, \quad \epsilon_{0}>0
$$

onde $\langle x\rangle=\left(1+x^{2}\right)^{1 / 2}$, como para $r \geq 1$,

$$
\frac{r(1+\log r)^{2}}{\left(1+r^{2}\right)^{\frac{1}{2}+\epsilon_{0}}} \leq \frac{(1+\log r)^{2}}{r^{2 \epsilon_{0}}}
$$

podemos mostrar que existe uma constante

$$
C\left(\epsilon_{0}\right)= \begin{cases}1, & \text { se } \epsilon_{0} \geq 1 \\ \frac{1}{\epsilon_{0}^{2} e^{2\left(1-\epsilon_{0}\right)},}, & \text { se } \epsilon_{0}<1\end{cases}
$$

tal que

$$
\left|V_{1}(x, t)\right|^{2} \leq \frac{c_{1}^{2} C\left(\epsilon_{0}\right)}{r(1+\log r)^{2}}, \quad r \geq 1 .
$$

Portanto, combinando as desigualdades (A.21) e (A.23), mantendo $r \geq 1$ e também $\lambda \geq$ $\lambda_{2}^{\prime}=\max \left\{\lambda_{1}^{\prime}, \frac{1+\sqrt{1+64\left(14+5 e^{-1}+c_{1}^{2} C\left(\epsilon_{0}\right)\right)}}{32}\right\}$, conseguimos que

$$
\begin{aligned}
4 \lambda^{3}\left(\psi^{\prime}(r)\right)^{2} \psi^{\prime \prime}(r)-\lambda \psi^{(i v)}(r)-\left|V_{1}\right|^{2} & \geq \frac{16 \lambda^{2}-\left(14+5 e^{-1}\right)-c_{1}^{2} C\left(\epsilon_{0}\right)}{r(1+\log r)^{2}} \\
& \geq \frac{\lambda}{r(1+\log r)^{2}} .
\end{aligned}
$$

Devido a que a primeira derivada $\psi^{\prime}$ é crescente no intervalo $[0,1]$ e o valor mínimo da segunda derivada $\psi^{\prime \prime}$ é 1 sobre $[0,1]$, encontramos

$$
\left[\psi^{\prime}(\epsilon)\right]^{2} \leq\left[\psi^{\prime}(r)\right]^{2} \psi^{\prime \prime}(r), \quad \epsilon \leq r \leq 1
$$

para qualquer $\epsilon$ entre zero e um. Além disso, $\max \left\{\psi^{(i v)}(r): \epsilon \leq r \leq 1\right\}=\psi^{(i v)}(1)=14$. Então, vemos que para

$$
\lambda \geq \lambda_{3}^{\prime}=\max \left\{1, \frac{\sqrt{14}}{2 \psi^{\prime}(\epsilon)}, \frac{1+\sqrt{1+16\left(14+c_{1}\right)\left[\psi^{\prime}(\epsilon)\right]^{2}}}{8\left[\psi^{\prime}(\epsilon)\right]^{2}}\right\}
$$

se satisfaz

$$
4 \lambda^{3}\left(\psi^{\prime}(r)\right)^{2} \psi^{\prime \prime}(r)-\lambda \psi^{(i v)}(r)-\left|V_{1}\right|^{2} \geq 4 \lambda^{2}\left[\psi^{\prime}(\epsilon)\right]^{2}-14-c_{1} \geq \lambda .
$$


Se assumimos uma função $V_{2}$ com suporte contido no conjunto $\{(x, t):|x| \geq 1\}$ e

$$
-\left(\partial_{r} V_{2}(x, t)\right)^{-} \leq \frac{c_{2}}{|x|^{\left(1+\epsilon_{0}\right)}}, \quad a^{-}=\min \{a ; 0\}, \quad \epsilon_{0}>0
$$

então

$$
-\frac{d \psi}{d r}\left(\partial_{r} V_{2}(x, t)\right)^{-} \leq \frac{c_{2} c\left(\epsilon_{0}\right)}{|x|(1+\log |x|)^{2}}, \quad|x| \geq 1
$$

onde

$$
c\left(\epsilon_{0}\right)=\left\{\begin{array}{ll}
1, & \text { se } \epsilon_{0} \geq 2 \\
\frac{4}{\epsilon_{0}^{2} e^{\left(2-\epsilon_{0}\right)}}, & \text { se } \epsilon_{0}<2
\end{array},\right.
$$

Assim, para $\lambda \geq \lambda_{4}^{\prime}=\sqrt{\frac{14+5 e^{-1}+2\left(c\left(\epsilon_{0}\right) c_{2}\right.}{16}}$ e $|x| \geq 1$, temos

$$
\begin{aligned}
4 \lambda^{3}\left(\psi^{\prime}\right)^{2} \psi^{\prime \prime}-\lambda \psi^{(i v)}-\left|V_{1}\right|^{2} & \\
& +2 \lambda \frac{d \psi}{d r}\left(\partial_{r} V_{2}(x, t)\right)^{-} \geq \frac{16 \lambda^{2}-\left(14+5 e^{-1}\right)-2\left(c\left(\epsilon_{0}\right) c_{2}-c_{1}^{2}\right.}{|x|(1+\log |x|)^{2}} .
\end{aligned}
$$

Logo,

$$
4 \lambda^{3}\left(\psi^{\prime}\right)^{2} \psi^{\prime \prime}-\lambda \psi^{(i v)}-\left|V_{1}\right|^{2}+2 \lambda \frac{d \psi}{d r}\left(\partial_{r} V_{2}(x, t)\right)^{-} \geq \frac{\lambda}{|x|(1+\log |x|)^{2}},
$$

sempre que $\lambda \geq \lambda_{5}^{\prime}=\max \left\{\lambda_{4}^{\prime}, \frac{1+\sqrt{1+64\left(14+5 e^{-1}+2\left(c\left(\epsilon_{0}\right) c_{2}+c_{1}^{2}\right)\right.}}{32}\right\}$ e $|x| \geq 1$. 


\section{Apêndice B}

\section{Demonstração da Proposição 3.3 e do Corolário 3.5}

Neste apêndice estabelecemos algumas demonstrações de afirmações feitas no texto.

\section{B.1 Demostração da Proposição 3.3}

Como sabemos toda função $\vartheta \in W^{m, p}(\mathbb{R})$ pode ser aproximada por funções no espaço $C_{0}^{\infty}(\mathbb{R})$, e o que mostraremos é que se dita função anula-se em zero, então podemos aproximala por funções no espaço $C_{0}^{\infty}(\mathbb{R})$ cujo suporte não contem a zero. O que muda nesta demonstração com relação à demonstração da afirmação clássica, é a escolha das sequências de funções de cortes e regularizantes. Seguidamente, aproximarmos cada uma essas funções por funções de teste em $\mathbb{R}$.

Seja $\vartheta \in W^{m, p}(\mathbb{R})$ e consideremos para cada $n \in \mathbb{Z}^{+}$, a função $\varphi_{n} \in C_{0}^{\infty}(\mathbb{R})$ tal que $0 \leq \varphi_{n}(x) \leq 1$ para todo $x \in \mathbb{R}$ e além disso,

$$
\varphi_{n}(x)=\left\{\begin{array}{lll}
1, & \text { se } & \frac{2}{n^{2}} \leq|x| \leq 2 \\
0, & \text { se }|x|<\frac{1}{n^{2}} \quad \text { ou } \quad|x|>3 .
\end{array}\right.
$$

Definamos as funções $\xi_{n}(x)=\varphi_{n}\left(\frac{x}{n}\right)$, para cada $n \in \mathbb{Z}^{+}$. Assim, $\left(\xi_{n}\right)_{n \geq 1}$ é uma sequência de funções teste satisfazendo:

a) $\xi_{n}=1$ sobre $\left\{x \in \mathbb{R}: \frac{2}{n} \leq|x| \leq 2 n\right\}, \operatorname{supp}\left(\xi_{n}\right) \subset\left\{x \in \mathbb{R}: \frac{1}{n} \leq|x| \leq 3 n\right\}$ e $0 \leq \xi_{n}(x) \leq 1$

b) Para todo $r \in \mathbb{N}$, temos $D^{r} \xi_{n}(x)=\frac{1}{n^{r}} D^{r} \varphi_{n}\left(\frac{x}{n}\right)$.

Aplicando a fórmula de Leibniz a cada função $\xi_{n} \vartheta$, para cada $n \in \mathbb{Z}^{+}$, o Teorema da Convergência Dominada de Lebesgue e os fatos dados nos item (a) e (b) acima, obtemos que a sequência $\left(\xi_{n} \vartheta\right)_{n \geq 1}$ converge a $\vartheta$ na norma de $W^{m, p}(\mathbb{R})$.

Cabe notar que cada função $\vartheta_{n}=\xi_{n} \vartheta$ possui suporte compacto contido no conjunto $\mathbb{R} \backslash\left(-\frac{1}{n}, \frac{1}{n}\right)$.

Agora, tomemos una sequência $\left(\rho_{k}\right)_{k \geq 1}$ regularizante no $\mathbb{R}$ tal que $\operatorname{supp}\left(\rho_{k}\right) \subset\left(-\frac{1}{k}, \frac{1}{k}\right)$. Segue que para cada $n \in \mathbb{N}$, os termos da sequência $\left(\rho_{k} * \vartheta_{n}\right)_{k \geq 1}$ são funções testes no $\mathbb{R}$. Além disso, para cada $r \leq m$, temos

$$
D^{r}\left(\rho_{k} * \vartheta_{n}\right)=\rho_{k} * D^{r} \vartheta_{n}
$$


e como também é verdade que $\rho_{k} * v \rightarrow v$ em $L^{p}(\mathbb{R})$ para todo $v \in L^{p}(\mathbb{R})$ conseguimos que

$$
D^{r}\left(\rho_{k} * \vartheta_{n}\right)=\rho_{k} * D^{r} \vartheta_{n} \rightarrow D^{r} \vartheta_{n}
$$

em $L^{p}(\mathbb{R})$, quando $k \longrightarrow \infty$, ficando provado que

$$
\rho_{k} * \vartheta_{n} \rightarrow \vartheta_{n}
$$

em $W^{m, p}(\mathbb{R})$, quando $k \longrightarrow \infty$. Até aqui encontramos uma sequência $\left(\rho_{k} * \vartheta_{n}\right)_{k \geq 1, n \geq 1}$ no espaço $C_{0}^{\infty}(\mathbb{R})$, da qual extraímos a subsequência $\left(\psi_{n}=\rho_{n} * \vartheta_{n}\right)_{n \geq 1}$ que vai satisfazer as condições pedidas, pois $\psi_{n} \rightarrow \vartheta$ em norma $W^{m, p}(\mathbb{R})$ e

$$
\psi_{n}(0)=\int_{\mathbb{R}} \rho_{n}(y) \vartheta_{n}(-y) d y=\int_{\operatorname{supp}\left(\rho_{n}\right)} \rho_{n}(y) \vartheta_{n}(-y) d y=0,
$$

porque $\vartheta_{n} \equiv 0$ sobre o suporte de $\rho_{n}$.

\section{B.2 Demonstração do Corolário 3.5}

Iniciamos com seguinte lema:

Lema B.1 Sejam $1 \leq p<\infty$ e $1 \leq q<\infty$. Se $f \in L^{q}\left(\mathbb{R}: L^{p}(\mathbb{R})\right)$ e $s_{0} \in \mathbb{R}$, então

$$
\lim _{s \rightarrow 0}\left(\int\left\|f\left(t-\left(s+s_{0}\right)\right)-f\left(t-s_{0}\right)\right\|_{L^{p}}^{q} d t\right)^{\frac{1}{q}}=0
$$

Demonstração. Vamos primeiro supor que $s_{0}=0$. Seja $g \in C\left(\mathbb{R}: L^{q}(\mathbb{R})\right)$ de suporte compacto. Para $|s| \leq 1$ as funções $g(\cdot-s)$ são todas suportadas num conjunto compacto comum $K$. Logo, para $\epsilon>0$, existe $\delta>0$ tal que

$$
\int\|g(t-s)-g(t)\|_{L^{p}}^{q} d t \leq \sup \|g(t-s)-g(t)\|_{L^{p}}^{q} \mu(K)<\frac{\epsilon}{3}, \quad \text { sempre que } \quad|s|<\delta
$$

Agora, suponha que $f \in L^{q}\left(\mathbb{R}: L^{p}(\mathbb{R})\right)$. Portanto, para $\epsilon>0$ dado, existe $g \in C_{0}\left(\mathbb{R}: L^{q}(\mathbb{R})\right)$ com

$$
\left(\int\|f(t)-g(t)\|_{L^{p}}^{q} d t\right)^{\frac{1}{q}}<\frac{\epsilon}{3}
$$

Então, para $\epsilon>0$ e $\delta>0$ anteriores, temos

$$
\begin{aligned}
\left(\int\|f(t-s)-f(t)\|_{L^{p}}^{q} d t\right)^{\frac{1}{q}} & \leq\left(\int\|f(t-s)-g(t-s)\|_{L^{p}}^{q} d t\right)^{\frac{1}{q}} \\
& +\left(\int\|g(t-s)-g(t)\|_{L^{p}}^{q} d t\right)^{\frac{1}{q}}+\left(\int\|g(t)-f(t)\|_{L^{p}}^{q} d t\right)^{\frac{1}{q}} \\
<\epsilon, &
\end{aligned}
$$

sempre que $|s|<\delta$.

Demonstração de Corolário 3.6. Pela continuidade uniforme da função $u \in C([A, B]$ : $\left.D\left(-\Delta_{Z}\right)\right) \cap C^{1}\left([A, B]: L^{2}(\mathbb{R})\right)$, para cada $n \in \mathbb{Z}^{+}$, existe $\delta>0$ tal que

$$
\|u(t)-u(s)\|_{H^{2}(\mathbb{R} \backslash\{0\})}<\frac{1}{n^{2}}, \quad \text { sempre que } \quad|t-s|<\delta .
$$


Agora, escolha $m_{n} \in \mathbb{Z}^{+}$tal que $m_{n}>n, m_{n}<m_{n+1}$ e $(B-A) / m_{n}<\delta$ e consideremos a partição do intervalo $[A, B]$ com pontos de divisão:

$$
t_{j}=A+(j-1) \frac{B-A}{m_{n}}, \quad j=1, \ldots, m_{n}+1 .
$$

Por outro lado, como $\mathbf{Y}$ é denso em $D\left(-\Delta_{Z}\right)$, existem funções $\omega_{m_{n}}(t)$ definidas em $[A, B]$ com imagem em $\mathbf{Y}$ satisfazendo

$$
\left\|\omega_{m_{n}}(t)-u(t)\right\|_{H^{2}(\mathbb{R} \backslash\{0\})}<\frac{1}{m_{n}^{2}} .
$$

Agora, definamos as funções

$$
\Theta_{m_{n}}(x, t)=\sum_{j=1}^{m_{n}+1} \gamma_{j-1}(x, t) \chi_{\left[t_{j-1}, t_{j}\right)}(t)
$$

onde os $t_{j}$ 's são como em (B.2) e

$$
\gamma_{j-1}(x, t)=\frac{\omega_{m_{n}}\left(x, t_{j}\right)-\omega_{m_{n}}\left(x, t_{j-1}\right)}{t_{j}-t_{j-1}}\left(t-t_{j-1}\right)+\omega_{m_{n}}\left(x, t_{j-1}\right) .
$$

Temos construído a sequência de funções $\left(\Theta_{m_{n}}\right)_{n \geq 1}, \Theta_{m_{n}}(t) \in \mathbf{Y}$, que verifica o seguinte:

(i) Para cada $t \in[A, B],\left\|u(t)-\Theta_{m_{n}}(t)\right\|_{H^{2}(\mathbb{R} \backslash\{0\})} \rightarrow 0$, quando $n \rightarrow \infty$. Com efeito, para $t \in\left[t_{j-1}, t_{j}\right)$

$$
\begin{aligned}
\| u(t)- & \Theta_{m_{n}}(t) \|_{H^{2}(\mathbb{R} \backslash\{0\})} \\
& =\left\|\frac{\omega_{m_{n}}\left(t_{j}\right)-\omega_{m_{n}}\left(t_{j-1}\right)}{t_{j}-t_{j-1}}\left(t-t_{j-1}\right)+\omega_{m_{n}}\left(t_{j-1}\right)-u(t)\right\|_{H^{2}(\mathbb{R} \backslash\{0\})} \\
& \leq\left\|\omega_{m_{n}}\left(t_{j}\right)-u(t)\right\|_{H^{2}(\mathbb{R} \backslash\{0\})}+2\left\|\omega_{m_{n}}\left(t_{j-1}\right)-u(t)\right\|_{H^{2}(\mathbb{R} \backslash\{0\})} \\
& \leq\left\|\omega_{m_{n}}\left(t_{j}\right)-u\left(t_{j}\right)\right\|_{H^{2}(\mathbb{R} \backslash\{0\})}+\left\|u\left(t_{j}\right)-u(t)\right\|_{H^{2}(\mathbb{R} \backslash\{0\})} \\
& +2\left\|\omega_{m_{n}}\left(t_{j-1}\right)-u\left(t_{j-1}\right)\right\|_{H^{2}(\mathbb{R} \backslash\{0\})}+2\left\|u\left(t_{j-1}\right)-u(t)\right\|_{H^{2}(\mathbb{R} \backslash\{0\})} \\
& <\frac{6}{n^{2}}
\end{aligned}
$$

(ii) Do Teorema da Convergência Dominada, $\int_{A}^{B}\left\|u(t)-\Theta_{m_{n}}(t)\right\|_{H^{2}(\mathbb{R} \backslash\{0\})} d t \rightarrow 0$, quando $n \rightarrow \infty$.

Agora, consideremos uma extensão da função $\Gamma_{n}$ definida em $\mathbb{R} \times \mathbb{R}$, como

$$
\Gamma_{m_{n}}(x, t)= \begin{cases}\Theta_{m_{n}}(x, t), & \text { se }(x, t) \in \mathbb{R} \times[A, B] \\ 0, & \text { em outro caso }\end{cases}
$$

Continuamos com a regularização de cada uma das funções $\Gamma_{m_{n}}$ com respeito à variável temporal $t$, escolhendo uma função teste $\tau \in C_{0}^{\infty}(\mathbb{R})$, que tem a propriedade $\int \tau(t) d t=1$, e definindo $\tau_{r}(t)=r \tau(r t)$. Então, pelo Lema B.1, a sequência $\left(\Gamma_{m_{n}} * \tau_{r}\right)_{r \geq 1}$ contida no espaço das funções $C_{0}^{\infty}(\mathbb{R}: \mathbf{Y})$ e satisfaz

(iii) Para cada $t \in[A, B]$, temos $\left\|\Gamma_{m_{n}} * \tau_{r}(t)-\Gamma_{m_{n}}(t)\right\|_{H^{2}(\mathbb{R} \backslash\{0\})} \rightarrow 0$, quando $r \rightarrow \infty$,

(iv) $\int_{A}^{B}\left\|\Gamma_{m_{n}} * \tau_{r}(t)-\Gamma_{m_{n}}(t)\right\|_{H^{2}(\mathbb{R} \backslash\{0\})} d t \rightarrow 0$, quando $r \rightarrow \infty$. 
Portanto de (i)-(iv) obtemos que a sequência $\left(\Gamma_{m_{n}} * \tau_{n}\right)_{n \geq 1}$

(v) Para cada $t \in[A, B]$, temos $\left\|\Gamma_{m_{n}} * \tau_{n}(t)-u(t)\right\|_{H^{2}(\mathbb{R} \backslash\{0\})} \rightarrow 0$, quando $n \rightarrow \infty$,

(vi) $\int_{A}^{B}\left\|\Gamma_{m_{n}} * \tau_{n}(t)-u(t)\right\|_{H^{2}(\mathbb{R} \backslash\{0\})} d t \rightarrow 0$, quando $n \rightarrow \infty$

Também, já que o suporte $\Gamma_{m_{n}}(t)$ e $u(t)$ é o intervalo $[A, B]$, e o suporte de $u\left(t+\frac{B-A}{m_{n}}\right)$ é $\left[A-\frac{B-A}{m_{n}}, B-\frac{B-A}{m_{n}}\right]$ então, o suporte de $\partial_{t} \Gamma_{m_{n}} * \tau_{n}(t)$ e $u\left(\cdot+\frac{B-A}{m_{n}}\right) * \tau_{m_{n}}(t)$ está contido no intervalo $\left[A-\frac{B-A+1}{m_{n}}, B+\frac{1}{m_{n}}\right]$, logo

$$
\begin{gathered}
\int\left\|\int \partial_{t} \Gamma_{m_{n}}(t-s) \tau_{m_{n}}(s) d s-\int \frac{u\left(t-s+\frac{(B-A)}{m_{n}}\right)-u(t-s)}{\frac{(B-A)}{m_{n}}} \tau_{m_{n}}(s) d s\right\| d t \\
=\int \| \int\left(\sum_{j-2}^{n+1} \frac{\omega_{m_{n}}\left(t_{j}\right)-\omega_{m_{n}}\left(t_{j-1}\right)}{t_{j}-t_{j-1}} \chi_{\left[t_{j-1}, t_{j}\right)}(t-s)\right. \\
-\frac{u\left(t-s+\frac{(B-A)}{m_{n}}\right)-u(t-s)}{\left.\frac{(B-A)}{m_{n}}\right) \tau_{m_{n}}(s) d s \| d t} \\
\leq \int_{A-\frac{B-A+1}{m_{n}}}^{B+\frac{1}{m_{n}}} \sum_{j-2}^{m_{n}+1} \int_{t-t_{j}}^{t-t_{j-1}} \tau_{m_{n}}(s)\left(\frac{\left\|\omega_{m_{n}}\left(t_{j}\right)-u\left(t-s+\frac{(B-A)}{m_{n}}\right)\right\|}{t_{j}-t_{j-1}}+\right. \\
\left.+\frac{\left\|\omega_{m_{n}}\left(t_{j-1}\right)-u(t-s)\right\|}{\frac{(B-A)}{m_{n}}}\right) d s d t \\
<\frac{m_{n}}{(B-A)} \frac{2}{m_{n}^{2}}\left(B-A+\frac{(B-A)+2}{m_{n}}\right) \leq \frac{(B-A)+2}{(B-A) m_{n}} .
\end{gathered}
$$

Portanto,

$$
\int\left\|\int \partial_{t} \Gamma_{m_{n}}(t-s) \tau_{m_{n}}(s) d s-\int \frac{u\left(t-s+\frac{(B-A)}{m_{n}}\right)-u(t-s)}{\frac{(B-A)}{m_{n}}} \tau_{m_{n}}(s) d s\right\| d t \rightarrow 0
$$

quando $n \rightarrow \infty$.

Por outro lado, para $\epsilon>0$ obtemos

$$
\begin{aligned}
& \int\left\|\int \frac{u\left(t-s+\frac{(B-A)}{m_{n}}\right)-u(t-s)}{\frac{(B-A)}{m_{n}}} \tau_{m_{n}}(s) d s-\partial_{t}\left(u * \tau_{m_{n}}\right)(t)\right\| d s d t \\
& \leq \int \tau_{m_{n}}(s) \int\left\|\frac{u\left(t-s+\frac{(B-A)}{m_{n}}\right)-u(t-s)}{\frac{(B-A)}{m_{n}}}-\partial_{t} u(t-s)\right\| d t d s \\
& <\epsilon \int \tau_{m_{n}}(s) d s=\epsilon,
\end{aligned}
$$

a partir de um $n \in \mathbb{Z}^{+}$suficientemente grande. 
Já que

$$
\begin{aligned}
\int\left\|\partial_{t}\left(\Gamma_{m_{n}} * \tau_{m_{n}}\right)(t)-\partial_{t} u(t)\right\| d t & \leq \int \| \partial_{t}\left(\Gamma_{m_{n}} * \tau_{m_{n}}\right)(t) \\
-\int \frac{u\left(t-s+\frac{(B-A)}{m_{n}}\right)-u(t-s)}{\frac{(B-A)}{m_{n}}} \tau_{m_{n}}(s) d s \| d t & \\
& +\int\left\|\int \frac{u\left(t-s+\frac{(B-A)}{m_{n}}\right)-u(t-s)}{\frac{(B-A)}{m_{n}}} \tau_{m_{n}}(s) d s-\partial_{t}\left(u * \tau_{m_{n}}\right)(t)\right\| d t \\
& +\int\left\|\partial_{t}\left(u * \tau_{m_{n}}\right)(t)-\partial_{t} u(t)\right\| d t
\end{aligned}
$$

de (B.4) e (B.5) obtemos

- $\int\left\|\partial_{t}\left(\Gamma_{m_{n}} * \tau_{m_{n}}\right)(t)-\partial_{t} u(t)\right\| d t \rightarrow 0$, quando $n \rightarrow \infty$.

\section{B.3 Demonstração da identidade 3.17}

Aqui demonstraremos a identidade,

$$
\begin{aligned}
\langle\mathcal{S} \xi, \mathcal{A} \xi\rangle & +\langle\mathcal{A} \xi, \mathcal{S} \xi\rangle=2 Z \lambda \varphi^{\prime \prime}(0)|\xi(0)|^{2} \\
& +\int\left(4 \lambda \varphi^{\prime \prime}\left|\partial_{x} \xi\right|^{2}-\lambda \varphi^{(i v)}|\xi|^{2}+4 \lambda^{3}\left(\varphi^{\prime}\right)^{2} \varphi^{\prime \prime}|\xi|^{2}\right) d x .
\end{aligned}
$$

O símbolo da integral $\int$ significará $\int_{\mathbb{R} \backslash\{0\}}$. Iniciamos com a relação,

$$
\begin{aligned}
-\frac{1}{\lambda}\langle\mathcal{S} \xi, \mathcal{A} \xi\rangle= & \int\left(2 \varphi^{\prime} \partial_{x} \xi+\varphi^{\prime \prime} \xi\right)\left(\partial_{x}^{2} \bar{\xi}+\lambda^{2}\left(\varphi^{\prime}\right)^{2} \bar{\xi}\right) d x \\
=2 \int \partial_{x} \xi \cdot \varphi^{\prime}\left(\partial_{x}^{2} \bar{\xi}+\lambda^{2}\left(\varphi^{\prime}\right)^{2} \bar{\xi}\right) d x & \\
& +\int\left(\varphi^{\prime \prime} \xi \partial_{x}^{2} \bar{\xi}+\lambda^{2}\left(\varphi^{\prime}\right)^{2} \varphi^{\prime \prime}|\xi|^{2}\right) d x
\end{aligned}
$$

Agora, integrando por partes, seja $\mu=\varphi^{\prime}\left(\partial_{x}^{2} \bar{\xi}+\lambda^{2}\left(\varphi^{\prime}\right)^{2} \bar{\xi}\right)$, então

$$
\begin{aligned}
d \mu / d x & =\varphi^{\prime \prime}\left(\partial_{x}^{2} \bar{\xi}+\lambda^{2}\left(\varphi^{\prime}\right)^{2} \bar{\xi}\right)+\varphi^{\prime}\left(\partial_{x}^{3} \bar{\xi}+2 \lambda^{2} \varphi^{\prime} \varphi^{\prime \prime} \bar{\xi}+\lambda^{2}\left(\varphi^{\prime}\right)^{2} \partial_{x} \bar{\xi}\right) \\
& =\varphi^{\prime \prime} \partial_{x}^{2} \bar{\xi}+3 \lambda^{2}\left(\varphi^{\prime}\right)^{2} \varphi^{\prime \prime} \bar{\xi}+\varphi^{\prime} \partial_{x}^{3} \bar{\xi}+\lambda^{2}\left(\varphi^{\prime}\right)^{3} \partial_{x} \bar{\xi},
\end{aligned}
$$

para $d \nu / d x=\partial_{x} \xi$, então $\nu=\xi$. Portanto,

$$
\begin{aligned}
-\frac{1}{\lambda}\langle\mathcal{S} \xi, \mathcal{A} \xi\rangle=- & 2 \int\left(\varphi^{\prime \prime} \xi \partial_{x}^{2} \bar{\xi}+3 \lambda^{2}\left(\varphi^{\prime}\right)^{2} \varphi^{\prime \prime}|\xi|^{2}+\varphi^{\prime} \xi \partial_{x}^{3} \bar{\xi}+\lambda^{2}\left(\varphi^{\prime}\right)^{3} \xi \partial_{x} \bar{\xi}\right) d x \\
& +\int\left(\varphi^{\prime \prime} \xi \partial_{x}^{2} \bar{\xi}+\lambda^{2}\left(\varphi^{\prime}\right)^{2} \varphi^{\prime \prime}|\xi|^{2}\right) d x \\
=- & \int\left(\varphi^{\prime \prime} \xi \partial_{x}^{2} \bar{\xi}+5 \lambda^{2}\left(\varphi^{\prime}\right)^{2} \varphi^{\prime \prime}|\xi|^{2}+2 \varphi^{\prime} \xi \partial_{x}^{3} \bar{\xi}+2 \lambda^{2}\left(\varphi^{\prime}\right)^{3} \xi \partial_{x} \bar{\xi}\right) d x
\end{aligned}
$$

De outro lado,

$$
\begin{aligned}
-\frac{1}{\lambda}\langle\mathcal{A} \xi, \mathcal{S} \xi\rangle= & \int\left(\partial_{x}^{2} \xi+\lambda^{2}\left(\varphi^{\prime}\right)^{2} \xi\right)\left(2 \varphi^{\prime} \partial_{x} \bar{\xi}+\varphi^{\prime \prime} \bar{\xi}\right) d x \\
= & \int \partial_{x}^{2} \xi\left(2 \varphi^{\prime} \partial_{x} \bar{\xi}+\varphi^{\prime \prime} \bar{\xi}\right) d x \\
& \quad+\int\left(2 \lambda^{2}\left(\varphi^{\prime}\right)^{3} \xi \partial_{x} \bar{\xi}+\lambda^{2}\left(\varphi^{\prime}\right)^{2} \varphi^{\prime \prime}|\xi|^{2}\right) d x
\end{aligned}
$$


Integrando por partes, com $\mu=2 \varphi^{\prime} \partial_{x} \bar{\xi}+\varphi^{\prime \prime} \bar{\xi}$, temos

$$
d \mu / d x=3 \varphi^{\prime \prime} \partial_{x} \bar{\xi}+2 \varphi^{\prime} \partial_{x}^{2} \bar{\xi}+\varphi^{\prime \prime \prime} \bar{\xi}
$$

para $d \nu / d x=\partial_{x}^{2} \xi$, temos $\nu=\partial_{x} \xi$. Por conseguinte,

$$
\begin{aligned}
-\frac{1}{\lambda}\langle\mathcal{A} \xi, \mathcal{S} \xi\rangle=\varphi^{\prime \prime} & (0) \bar{\xi}(0)\left[\partial_{x} \xi\left(0^{-}\right)-\partial_{x} \xi\left(0^{+}\right)\right] \\
& -\int \partial_{x} \xi\left(3 \varphi^{\prime \prime} \partial_{x} \bar{\xi}+2 \varphi^{\prime} \partial_{x}^{2} \bar{\xi}+\varphi^{\prime \prime \prime} \bar{\xi}\right) d x \\
& +\int\left(2 \lambda^{2}\left(\varphi^{\prime}\right)^{3} \xi \partial_{x} \bar{\xi}+\lambda^{2}\left(\varphi^{\prime}\right)^{2} \varphi^{\prime \prime}|\xi|^{2}\right) d x .
\end{aligned}
$$

Novamente integrando por partes, com $\mu=3 \varphi^{\prime \prime} \partial_{x} \bar{\xi}+2 \varphi^{\prime} \partial_{x}^{2} \bar{\xi}+\varphi^{\prime \prime \prime} \bar{\xi}$, temos

$$
d \mu / d x=4 \varphi^{\prime \prime \prime} \partial_{x} \bar{\xi}+5 \varphi^{\prime \prime} \partial_{x}^{2} \bar{\xi}+2 \varphi^{\prime} \partial_{x}^{3} \bar{\xi}+\varphi^{(i v)} \bar{\xi}
$$

para $d \nu / d x=\partial_{x} \xi$, temos $\nu=\xi, \log \mathrm{o}$

$$
\begin{aligned}
-\frac{1}{\lambda}\langle\mathcal{A} \xi, \mathcal{S} \xi\rangle & =-Z \varphi^{\prime \prime}(0)|\xi(0)|^{2}-3 \varphi^{\prime \prime}(0) \xi(0)\left[\partial_{x} \bar{\xi}\left(0^{-}\right)-\partial_{x} \bar{\xi}\left(0^{+}\right)\right] \\
+ & \int\left(4 \varphi^{\prime \prime \prime} \xi \partial_{x} \bar{\xi}+5 \varphi^{\prime \prime} \xi \partial_{x}^{2} \bar{\xi}+2 \varphi^{\prime} \xi \partial_{x}^{3} \bar{\xi}+\varphi^{(i v)}|\xi|^{2}\right) d x \\
& +\int\left(2 \lambda^{2}\left(\varphi^{\prime}\right)^{3} \xi \partial_{x} \bar{\xi}+\lambda^{2}\left(\varphi^{\prime}\right)^{2} \varphi^{\prime \prime}|\xi|^{2}\right) d x
\end{aligned}
$$

Somando (B.8) e (B.7), obtemos

$$
\begin{aligned}
-\frac{1}{\lambda}\langle\mathcal{S} \xi, \mathcal{A} \xi\rangle & -\frac{1}{\lambda}\langle\mathcal{A} \xi, \mathcal{S} \xi\rangle=2 Z \varphi^{\prime \prime}(0)|\xi(0)|^{2} \\
& +\int\left(4 \varphi^{\prime \prime \prime} \xi \partial_{x} \bar{\xi}+4 \varphi^{\prime \prime} \xi \partial_{x}^{2} \bar{\xi}+\varphi^{(i v)}|\xi|^{2}-4 \lambda^{2}\left(\varphi^{\prime}\right)^{2} \varphi^{\prime \prime}|\xi|^{2}\right) d x .
\end{aligned}
$$

Integrando por partes, com $\mu=\varphi^{\prime \prime} \xi$, então $d \mu / d x=\varphi^{\prime \prime \prime} \xi+\varphi^{\prime \prime} \partial_{x} \xi$ e parad $d \nu / d x=\partial_{x}^{2} \bar{\xi}$, temos $\nu=\partial_{x} \bar{\xi}$, assim

$$
\int \varphi^{\prime \prime} \xi \partial_{x}^{2} \bar{\xi} d x=-Z \varphi^{\prime \prime}(0)|\xi(0)|^{2}-\int\left(\varphi^{\prime \prime \prime} \xi \partial_{x} \bar{\xi}+\varphi^{\prime \prime}\left|\partial_{x} \xi\right|^{2}\right) d x
$$

e substituindo esta igualdade em (B.8), conseguimos

$$
\begin{aligned}
-\frac{1}{\lambda}\langle\mathcal{S} \xi, \mathcal{A} \xi\rangle & -\frac{1}{\lambda}\langle\mathcal{A} \xi, \mathcal{S} \xi\rangle=-2 Z \varphi^{\prime \prime}(0)|\xi(0)|^{2} \\
& +\int\left(-4 \varphi^{\prime \prime}\left|\partial_{x} \xi\right|^{2}+\varphi^{(i v)}|\xi|^{2}-4 \lambda^{2}\left(\varphi^{\prime}\right)^{2} \varphi^{\prime \prime}|\xi|^{2}\right) d x .
\end{aligned}
$$

O qual mostra a identidade (B.6). 


\section{Bibliografia}

[1] Adami, R., and Noja, D.; Existence of dynamics for a 1D NLS equation perturbed with a generalized point defect. J. Phys. A: Math Theor. 42, 1-19 (2009).

[2] Albeverio, S., Gesztesy, F., Hoegh-Krohn, R., and Holden, H.; Solvable Models in Quantum mechanics. Texts and Monographs in Physics. Springer-Verlag, New York (1988).

[3] Angulo, J. P., and Ferreira, L. C. F.; On the Schrödinger equations with singular potencials. Differential and Integral Equations 27, 767-800 (2014).

[4] Berezin, F. A., and Faddeev, L. D.; A remark on Schrödinger with a singular potencial. Soviet Math. Dokl. 2, 372-375 (1961).

[5] Cazenave, T.; Semilinear Schrödinger equations. Courant Lecture Note. AMS (2003)

[6] Conway, J. B.; A Course in Functional Analysis. Springer. 2nd ed. (1990).

[7] Datchev, K., and Holmer, J.; Fast solution scattering by attractive delta impurities. Commun. Partial Differ. Equations 34:9, 1074-1113 (2009) .

[8] Escauriaza, L., Kenig, C. E., Ponce, G, and Vega, L.; Unique Continuation for Schrödinger Evolutions, with Applications to profiles of Concentration and Traveling Waves. Commun. Math. Phys. 305, 487-512 (2011).

[9] Escauriaza, L., Kenig, C. E., Ponce, G, and Vega, L.; On Uniqueness Properties of Solutions of Schrödinger Equations. Comm. PDE. 31(12), 1811-1823 (2006) .

[10] Fukuizumi, R., and Jeanjean, L.; Stability of standing waves for a nonlinear schrödinger equation with a repulsive Dirac delta potencial. Discrete and Continuos Dynamical System, Vol. 21, Number 1, 121-136 (2008).

[11] Fukuizumi, R., Ohta, M., and Ozawa, T.; Nonlinear schrödinger equation with a point defect. Ann. I. H. Poincaré- AN25, 837-845 (2008).

[12] Hernandez Melo, C.; Estabilidade de ondas viajantes para equações de Schrödinger do tipo cúbica-quíntica. 2015. 135 f. Tese (Doutorado) - IME, Universidade de São Paulo, São Paulo, (2011).

[13] Ginibre, J., and Velo, G.; On a class of nonlinear Schrödinger equations. J. Funct. Anal. 32, 1-71 (1979).

[14] Holmer, J., Marzuola, J., and Zworski, M.; Fast solution scattering by delta impurities. Commun. Math. Phys. 274, 1, 187-216 (2007). 
[15] Le Coz, S., Fukuizumi, R., Fibich, G., Ksherim, B., and Sivan, Y.; Instability of bound states of a nonlinear Schrödinger equation with a Dirac potencial. Physica D. 273, 1103-1128 (2007).

[16] Pazy, A.; Semigroups of linear Operator an Applications to Partial Differential Equations. Springer Verlag Publishing (1983).

[17] Reed, M., and Simon, B.; Methods of modern mathematical physics. II:Fourier Analysis, Self-Adjointness. New york- San Francisco-London: Academic Press. XV (1978).

[18] Reed, M., and Simon, B.; Methods of modern mathematical physics. IV: Analysis of operators. New york- San Francisco-London: Academic Press. XV (1978).

[19] Stein, E.M., and Shakarchi,R.; Princeton Lecture in Analysis II. Complex Analysis. Priscenton University Press (2003).

[20] Tang, S. H., and Zworski, M.; Potencial scattering on the real line. Lecture notes. Available at: https://math.berkeley.edu/ zworski/tz1.pdf (2005).

[21] Yajima, K.; Existence of Solutions for Schrödinger Evolution Equations. Commun. Math. Phys 110, 415-426 (1987). 